Publ. RIMS, Kyoto Univ. Ser. A

Vol. 3 (1968), pp. 51-130

\title{
A Classification of Factors
}

\author{
By
}

\author{
Huzihiro AraKI and E. J. Woods*t
}

\begin{abstract}
A classification of factors is given. For every factor $M$ we define an algebraic invariant $\mathrm{r}_{\infty}(M)$, called the asymptotic ratio set, which is a subset of the nonnegative real numbers. For factors which are tensor products of type I factors, the set $\mathrm{r}_{\infty}(M)$ must be one of the following sets: (i) the empty set. (ii) $\{0\}$. (iii) $\{1\}$, (iv) a one-parameter family of sets $\left\{0, x^{n} ; n=0, \pm 1, \cdots\right\}$, $0<x<1$, (v) all nonnegative reals, (vi) $\{0,1\}$. Case (i), (ii), (iii) occurs if and only if $M$ is finite type $\mathrm{I}, \mathrm{I}_{\infty}$, hyperfinite type $\mathrm{II}_{1}$, respectively. Case (iv) contains one and only one isomorphic class for each $x$, and they are type III. The examples treated by Powers belong to case (iv). Case (v) contains only one isomorphic class and it is type III. Thus we have a complete classification of factors $M$ which are tensor products of type $I$ factors, $\mathrm{r}_{\infty}(M) \neq\{0,1\}$. Case (vi) contains $I_{\infty} \otimes$ hyperfinite II $_{1}$ and also nondenumerably many type III isomorphic classes.

Using the factors in the cases (ii), (iii), (iv) we define another algebraic invariant $\rho(M)$ which is able to distinguish nondenumerably many classes in case (vi).
\end{abstract}

\section{Introduction}

In the Murray-von Neumann classification of factors (Murray and von Neumann [11]) both the type $\mathrm{II}_{1}$ and type III classes are known to contain nonisomorphic factors. In this paper we give a further isomorphic classification of factors on separable Hilbert spaces. This classification is based on a detailed study of factors constructed as infinite tensor products of factors of finite type I (hereafter referred to as ITPFI factors). Examples of ITPFI factors were first given by von Neumann [12]. Several authors (von Neumann [12], Pukanszky [14], Bures [6], Araki [1], Moore [10]) have determined the type of

Received March 5, 1968 and, in revised form April 5, 1968.

* Department of Physics and Astronomy, University of Maryland, College Park, Maryland, U.S. A. Present address: Department of Mathematics, Queen's University, Kingston, Ontario, Canada.

† Supported in part by National Science Foundation Grant GP 6036. 
some of these factors in the Murray-von Neumann classification. Recently, Powers [13] has shown that these examples contain a oneparameter family of mutually nonisomorphic type III factors.

Sec. 2 contains some definitions and elementary lemmas concerning ITPFI factors. In Sec: 3 we define the asymptotic ratio set $\mathrm{r}_{\infty}(M) \subset$ $[0, \infty)$ for ITPFI factors $M=\otimes M_{\nu}$ in terms of ratios of eigenvalues $\lambda_{\nu j}$ of density matrix states $\omega_{\nu}$ on the component factors $M_{\nu}$. We show that $\mathrm{r}_{\infty}(M)$ must be one of the following standard sets

$$
\begin{aligned}
& S_{0}=\{0\} \\
& S_{1}=\{1\} \\
& S_{x}=\left\{0, x^{n} ; n=0, \pm 1, \pm 2, \cdots\right\}, 0<x<1 \\
& S_{01}=\{0,1\} \\
& S_{\infty}=[0, \infty) .
\end{aligned}
$$

We give some elementary properties of $\mathrm{r}_{\infty}(M)$, and discuss the oneparameter family of examples $R_{x}, 0 \leq x \leq 1$ given by von Neumann [12]. Sec. 4 consists of a basic technical lemma. In Sec. 5 we prove that $x \in \mathrm{r}_{\infty}\left(\otimes M_{\nu}\right)$ if and only if $\otimes M_{\nu} \sim R_{x} \otimes\left(\otimes M_{\nu}\right)$ and thus that $\mathrm{r}_{\infty}\left(\otimes M_{\nu}\right)$ is an algebraic invariant. Our method of proving that two factors are nonisomorphic is based entirely on the strong operator topology, in contrast to that of Powers [13] which uses $C^{*}$-algebra techniques. In Sec. 6 we use this result to define $\mathrm{r}_{\infty}(M)$ for arbitrary $M$ by $x \in \mathrm{r}_{\infty}(M)$ if and only $M \sim M \otimes R_{x}, 0 \leq x \leq 1$ (if $x \in \mathrm{r}_{\infty}(M), x \neq 0$ we include $x^{-1} \in \mathrm{r}_{\infty}(M)$ also). We give some elementary properties of $\mathrm{r}_{\infty}(M)$, including its relation to the Murray-von Neumann classification.

The remainder of the paper is devoted to a study of ITPFI factors. In Sec. 7 we prove that the class $S_{\infty}$ contains one and only one isomorphic class and it is type III. Sec. 8 contains a number of technical lemmas which are needed for the classification of ITPFI factors belonging to the classes $S_{x}, 0 \leq x \leq 1$. In Sec. 9 we prove that $\mathrm{r}_{\infty}(M)=S_{x}$ if and only if $M \sim R_{x}, 0 \leq x \leq 1$. The factors $R_{x}$ are type III if $0<x<1$, and they are the factors discussed by Powers 
[13]. Thus, except for the class $S_{01}$, we give a complete classification of factors which are tensor products of type I factors. We also give some useful criteria for calculating $\mathrm{r}_{\infty}(M)$ from the eigenvalue lists $\left\{\lambda_{\nu j} ; j=1, \cdots n_{\nu}, \nu=1,2, \cdots\right\}$. In particular a sufficient condition that $\mathrm{r}_{\infty}\left(\otimes M_{\nu}\right) \neq S_{01}$ is that there exist subsequences $\nu(m), j_{1}(m), j_{2}(m)$ such that $\lambda_{\nu(m), j_{1}(m)} \rightarrow \lambda_{i} \neq 0, i=1,2$ and $\lambda_{1} / \lambda_{2} \neq 1$. In Sec. 10 we study factors $M=\otimes M_{\nu}$ belonging to the class $S_{01}$ where $M_{\nu}$ is type $I_{2}$ for all $\nu . \quad M$ is then either $I_{\infty} \otimes$ hyperfinite $\mathrm{II}_{1}$ or type III. We construct a nondenumerable family of mutually nonisomorphic factors belonging to the class $S_{01}$. In Sec. 11 we define another algebraic invariant $\rho(M)$ by $x \in \rho(M)$ if and only if $M \otimes R_{x} \sim R_{x}, 0 \leqslant x \leqslant 1$. We construct factors in the class $S_{01}$ which give a nondenumerable variety of $\rho(M)$. In Sec. 12 we apply our results to determine the isomorphic class of some factors which have been studied previously in the literature. In particular we show that certain ITPFI factors which occur in the quantum theories of infinite free Bose and Fermi systems at a finite density and finite temperature, belong to the class $S_{\infty}$.

We shall use the following notation. If $H$ is a Hilbert space, then $\mathcal{B}(H)$ denotes the set of all bounded linear operators on $H$, and 1 denotes the set of all multiples of the identity operator. All Hilbert spaces are separable. $I_{\infty}$ denotes the set of all positive integers $\{1,2, \cdots\}$. We shall also use $I_{\infty}$ to denote a factor of type $I_{\infty}$, but this should not lead to any confusion. We assume that the reader is familiar with the standard notation and terminology for von Neumann algebras (Dixmier [8]). If the von Neumann algebras $\mathfrak{A}$ and $\mathfrak{B}$ are algebraically isomorphic (unitarily equivalent) we write $\mathfrak{A} \sim \mathfrak{B}(\mathfrak{A} \stackrel{\mathfrak{u}}{\sim} \mathfrak{B})$.

\section{ITPFI Factors}

This section contains some basic definitions and elementary lemmas concerning ITPFI factors. We discuss the notion of the eigenvalue list of a vector relative to a type I factor, and some related topics. We give a sufficient condition on the eigenvalue lists of the reference vectors for two ITPFI factors to be unitarily equivalent. We state 
some known results concerning the type of ITPFI factors in the Murray-von Neumann classification.

A family of matrix units on a Hilbert space $H$ is a set of partial isometries $e_{i j}, i, j=1, \cdots n$ ( $n$ may be infinite) satisfying $e_{i j}^{*}=e_{j i}$, $e_{i j} e_{k l}=\delta_{j k} e_{i l}$, and $\sum_{i=1}^{n} e_{i i}=1$. Any type $I_{n}$ factor contains and is spanned by such a family of matrix units.

Let $H$ be a Hilbert space, $M \subset \mathscr{B}(H)$ a type I factor. Then we can write $H=H_{1} \otimes H_{2}$ and $M=\mathscr{B}\left(H_{1}\right) \otimes 1$. If $\Omega$ is a vector in $H$ then it defines a normal state on $\mathcal{B}\left(H_{1}\right)$ by

$$
\Omega(A)=(\Omega, A \otimes 1 \Omega) .
$$

Hence there exists a nonnegative trace class operator $\rho_{2} \in \mathscr{B}\left(H_{1}\right)$ such that $\Omega(A)=\operatorname{Tr} \rho_{\Omega} A$. Let $\rho_{\Omega}=\sum \lambda_{i} P_{i}$ be a spectral decomposition of $\rho_{\Omega}$ where each $P_{i}$ is one-dimensional, $\lambda_{i} \geq 0$ and $\sum \lambda_{i}=\|\Omega\|_{i}^{2}$. If $\Omega$ is a unit vector then $\rho_{\Omega}$ is a density matrix, that is $\operatorname{Tr} \rho_{\Omega}=1$.

Definition 2.1. Let $\Omega \in H_{1} \otimes H_{2}, \quad M=\mathscr{B}\left(H_{1}\right) \otimes 1$. By the eigenvalue list of $\Omega$ relative to a type I factor $M$ we mean the list $\left(\lambda_{1}, \lambda_{2} \cdots\right)$ of eigenvalues of the operator $\rho_{2.2}$ in $M$ defined by

$$
\operatorname{Tr} \rho_{\Omega} A=(\Omega, A \otimes 1 \Omega)
$$

ordered so that $\lambda_{1} \geq \lambda_{2} \geq \cdots \geq 0$. We denote it by $\operatorname{Sp}(\Omega / M)$, or $\operatorname{Sp} \Omega$ if $M$ is understood.

If some $\lambda$ has multiplicity $m$ then it occurs $m$ times in $\operatorname{Sp}(\Omega / M)$. It should be noted that $\operatorname{Sp}(\Omega / M)$ and $\operatorname{Sp}\left(\Omega / M^{\prime}\right)$ are identical except that the zero eigenvalue can have different multiplicity.

Definition 2.2. Given $H=H_{1} \otimes H_{2}, M=\mathscr{B}\left(H_{1}\right) \otimes 1, \Omega \in H$. By a standard diagonal expansion of $\Omega$ relative to $M$ we mean a choice of complete orthogonal bases $\psi_{1 i}, \psi_{2 i}$ for $H_{1}, H_{2}$ respectively such that

$$
\Omega=\sum \lambda_{i}^{1 / 2} \psi_{1 i} \otimes \psi_{2 i}
$$

where $\lambda_{1} \geq \lambda_{2} \geq \cdots \geq 0, \psi$ 's in one of $\left\{\psi_{1 i}\right\}$ and $\left\{\psi_{2 i}\right\}$ are all normalized, $\psi$ 's in the other are normalized or 0 , and $\lambda_{1}=0$ if $\psi_{1 i}$ or $\psi_{2 i}=0$.

It is known that a standard diagonal expansion exists (see, e.g. 
definition 2.1 of $[2])$. Note that the list of non-zero $\lambda_{i}$ is identical with the non-zero part of $\operatorname{Sp}(\Omega / M)$.

Definition 2. 3. Given $H=H_{1} \otimes H_{2}, M=\mathscr{B}\left(H_{1}\right) \otimes \mathbb{1}, \Omega \in H$. By a standard set of matrix units for $M, M^{\prime}$ relative to $\Omega$ we mean operators $u_{i j}, v_{i j}$ defined by

$$
\begin{aligned}
& u_{i j} \psi_{1 k} \otimes \psi_{2 l}=\delta_{j k} \psi_{r_{i}} \otimes \psi_{2 l} \\
& v_{i j} \psi_{1 k} \otimes \psi_{2 l}=\delta_{j l} \psi_{r_{1}} \otimes \psi_{2 i}
\end{aligned}
$$

where $\psi_{1 i}, \psi_{2 i}$ is a choice of orthonormal bases for $H_{1}, H_{2}$ respectively for a standard diagonal expansion of $\Omega$ relative to $M$. If $\psi_{1 i}=0$ for some $i$, we define $u_{i j}$ and $u_{j i}$ for such $i$ and any $j$ to be 0 . If $\psi_{2 i}=$ 0 for some $i$, we define $v_{i j}$ and $v_{j i}$ for such $i$ and any $j$ to be 0 . We identify $\operatorname{Sp}(\Omega / M)$ with the set of $\lambda_{i}$ for which $\psi_{1 i} \neq 0$ and $\operatorname{Sp}\left(\Omega / M^{\prime}\right)$ with the set of $\lambda_{i}$ for which $\psi_{2 i} \neq 0$ in (2.3).

We now give a precise definition of an ITPFI factor. Let

$$
H=\bigotimes_{\nu \in A}\left(H_{\nu}, \Omega_{\nu}\right)
$$

be the incomplete tensor product space (ITPS) of the Hilbert spaces $H_{\nu}$ which contains the product vector $\Omega=\otimes \Omega_{\nu}, \Omega_{\nu} \in H_{\nu}, 0<\Pi\left\|\Omega_{\nu}\right\|<\infty$. In this paper the index set $A$ is always countable. If $\Omega$ and $A$ are understood we just write $H=\otimes H_{\nu}$. We assume the reader is familiar with the standard properties of infinite tensor products (von Neumann $[12])$. We note that $\otimes x_{\nu}$ belongs to the same ITPS as $\otimes \Omega_{\nu}$ if and only if $\otimes x_{\nu}$ is in the strong equivalence class of $\otimes \Omega_{\nu}$, that is

$$
\sum_{\nu \in A}\left\|\Omega_{\nu}-\chi_{\nu}\right\|<\infty
$$

This is equivalent to

$$
\sum\left|1-\left(\chi_{\nu}, \Omega_{\nu}\right)\right|<\infty \text { and } \sum\left|1-\left\|\chi_{\nu}\right\|\right|<\infty
$$

In both cases, $0<\Pi\left\|\Omega_{\nu}\right\|<\infty$ is assumed.

Definition 2.4. We define a canonical mapping $\pi$ from $\mathcal{B}\left(H_{\nu}\right)$ to $\mathscr{B}(H)$ by $\pi S=\left(\bigotimes_{\mu \neq \nu} 1_{\mu}\right) \otimes S$ where $S \in B\left(H_{\nu}\right)$ and $1_{\mu}$ is the identity operator on $H_{\mu}$. If $\mathfrak{A} \subset \mathscr{B}\left(H_{\nu}\right)$ we define $\pi \mathfrak{A}=\{\pi S: S \in \mathfrak{A}\}$. 
Definition 2.5. Given an ITPS $H=\bigotimes_{\nu \in A}\left(H_{\nu}, \Omega_{\nu}\right)$ and von Neumann algebras $\mathfrak{U}_{\nu} \subset \mathcal{B}\left(H_{\nu}\right)$ we define

$$
\otimes \mathfrak{A}_{\nu}=\left\{\pi \mathfrak{A}_{\nu} ; \nu \in A\right\}^{\prime \prime} .
$$

If the $\mathfrak{U}_{\nu}$ are factors, then $\otimes \mathfrak{U}_{\nu}$ is a factor. In the following we will be concerned with factors $\otimes M_{\nu}$ where $M_{\nu}$ is type $\mathrm{I}$. We shall denote these factors by $\mathrm{R}\left(H_{\nu}, M_{\nu}, \Omega_{\nu} ; \nu \in A\right)$ or $R\left(H_{\nu}, M_{\nu}, \Omega_{\nu}\right)$ or $\mathrm{R}\left(M_{\nu}, \Omega_{\nu}\right)$. Unless the contrary is stated explicitly, $M_{\nu}$ is type $I_{n_{\nu}}, 2 \leq n_{\nu}<\infty$, and $A$ is infinite. If $J \subset A$ we write $H(J)=\bigotimes_{\nu \in J} H_{\nu}, M(J)=\bigotimes_{\nu \in J} M_{\nu}, \Omega(J)=\bigotimes_{\nu \in J} \Omega_{\nu}$. If $\Omega_{\nu}$ and $\psi_{\nu}$ are in the same strong equivalence class, then $\otimes \psi_{\nu}$ is in $\otimes\left(H_{\nu}, \Omega_{\nu}\right)$ and hence $\mathrm{R}\left(H_{\nu}, M_{\nu}, \Omega_{\nu}\right)=\mathrm{R}\left(H_{\nu}, M_{\nu}, \psi_{\nu}\right)$. We shall use this repeatedly.

Definition 2.6. Any factor $M$ which is unitarily equivalent to some $\mathrm{R}\left(H_{\nu}, M_{\nu}, \Omega_{\nu} ; \nu \in A\right)$ as given above where $M_{\nu}$ is a type $I_{n_{\nu}}$ factor, $2 \leq n_{\nu}<\infty$ and $A$ is infinite is called an ITPFI factor.

We recall that a von Neumann algebra $M$ is called hyperfinite if it is generated by an increasing sequence $M_{1} \subset M_{2} \subset \cdots$ of finite type I factors, i.e.,

$$
M=\left\{M_{1}, M_{2}, \cdots\right\}^{\prime \prime}
$$

An ITPFI factor is clearly a hyperfinite factor. It is known that all hyperfinite factors of type $\mathrm{II}_{1}$ are isomorphic (Dixmier [8], theorem III. 7.1). Since an ITPFI factor is not finite type I, it must either be infinite or (isomorphic to) the unique hyperfinite $\mathrm{II}_{1}$ factor. We shall have several occasions to make use of this remark.

Lemma 2. 7. Let $\psi \in H=\bigotimes_{\nu \in A}\left(H_{\nu}, \Omega_{\nu}\right)$. Given $\varepsilon>0$, there exists a finite $J \subset A$ and $\psi_{J} \in H(J)$ such that

$$
\left\|\psi^{r}-\psi_{J} \otimes\left(\underset{\nu \in J^{\mathrm{c}}}{\otimes} \Omega_{\nu}\right)\right\|<\varepsilon .
$$

Proof. Araki and Woods [2], lemma 3.1.

Lemma 2. 8. A countable tensor product of ITPFI factors is an ITPFI factor. 
Proof. Let $M_{\mu}=\mathrm{R}\left(H_{\mu \nu}, M_{\mu \nu}, \Omega_{\mu \nu} ; \nu \in A_{\mu}\right), \mu \in A$ be ITPFI factors. Let $H_{\mu}=\bigotimes_{\nu \in A_{\mu}}\left(H_{\mu \nu}, \Omega_{\mu \nu}\right)$. Let $\Phi_{\mu}=\bigotimes_{\nu} \Omega_{\mu \nu}$, and

$$
\begin{aligned}
& H=\bigotimes_{\mu \in A}\left(H_{\mu}, \Phi_{\mu}\right) \\
& M=\bigotimes_{\mu \in A} M_{\mu} .
\end{aligned}
$$

Choose $\varepsilon_{\mu}>0, \sum \varepsilon_{\mu}<\infty$. By lemma 2.5 there is a finite $J_{\mu} \subset A_{\mu}$ and $\psi_{\mu} \in H\left(J_{\mu}\right)$ such that

$$
\left\|\Phi_{\mu}-\psi_{\mu} \otimes\left(\otimes_{\nu \in J_{\mu}^{c}} \Omega_{\mu \nu}\right)\right\|<\varepsilon_{\mu}
$$

Thus $\bigotimes_{\mu \in A}\left[\psi_{\mu} \otimes\left(\bigotimes_{\nu \in J_{\mu}} \Omega_{\mu \nu}\right)\right]$ is in the strong equivalence class of $\otimes \Phi_{\mu}$. It follows from the associative law for tensor products that $H$ is (unitarily equivalent to) the ITPS

$$
\left\{\bigotimes_{\mu \in A}\left(H\left(J_{\mu}\right), \psi_{\mu}\right)\right\} \otimes\left\{\bigotimes_{\mu \in A} \bigotimes_{\nu \in J_{\mu}^{\mathrm{c}}}\left(H_{\mu \nu}, \Omega_{\mu \nu}\right)\right\}
$$

Thus $M$ is an ITPFI factor.

Q.E.D.

Corollary 2.9. The factor $\mathrm{R}\left(H_{\nu}, M_{\nu}, \Omega_{\nu}\right)$ where $M_{\nu}$ can be type $I_{\infty}$ is an ITFFI factor.

Proof. Consider each type $I_{\infty}$ factor $M_{\nu}$ as an ITPFI factor and apply lemma 2.8 .

Q.E.D.

Lemma 2. 10. Given $H=H_{1} \otimes H_{2}, M=\mathscr{B}\left(H_{1}\right) \otimes 1$. Then $M$ has both cyclic vectors and separating vectors if and only if $\operatorname{dim} H_{1}=\operatorname{dim} H_{2}$. If $\operatorname{dim} H_{1}=\operatorname{dim} H_{2}<\infty$, let $\Omega \in H$ have the standard diagonal expansion $\Omega=\sum \lambda_{i}^{1 / 2} \psi_{1 i} \otimes \psi_{2 i}$. Then the following three conditions are equivalent.

(i) $\Omega$ is cyclic for $M$

(ii) $\Omega$ is separating for $(M)$

(iii) no $\lambda_{i}=0$.

Proof. Assume $\operatorname{dim} H_{2}>\operatorname{dim} H_{1}$. Since $M$ is spanned by $\left(\operatorname{dim} H_{1}\right)^{2}$ linearly independent elements, we have $\operatorname{dim} M \Omega \leq\left(\operatorname{dim} H_{1}\right)^{2}<\operatorname{dim} H$ and $\Omega$ cannot be cyclic for $M$. Similarly, $\operatorname{dim} H_{1}>\operatorname{dim} H_{2}$ implies $\Omega$ cannot be cyclic for $M^{\prime}$ and thus $\Omega$ is not separating for $M$. Thus 
the existence of both cyclic vectors and separating vectors for $M$ implies that $\operatorname{dim} H_{1}=\operatorname{dim} H_{2}$.

If $\operatorname{dim} H_{1}=\operatorname{dim} H_{2}$, then we may label complete orthonormal bases $\left\{\psi_{1 i}\right\}$ and $\left\{\psi_{2 i}\right\}$ of $H_{1}$ and $H_{2}$ respectively by the same index $i$, and Eq. (2.3) with $\lambda_{i}>0, \sum \lambda_{i}<\infty$ gives the cyclic and separating vector.

In the remainder of the proof we assume that $\operatorname{dim} H_{1}=\operatorname{dim} H_{2}<\infty$. If some $\lambda_{j}=0$ then $H_{1} \otimes \psi_{2 j}$ is orthogonal to $M \Omega$ and $\Omega$ is not cyclic for $M$, hence (i) $\rightarrow$ (iii). Similarly, if some $\lambda_{j}=0$ then $\psi_{1_{1 j}} \otimes H_{2}$ is orthogonal to $M^{\prime} \Omega$ and $\Omega$ is not cyclic for $M^{\prime}$. It follows that $\Omega$ is not separating for $M$, and hence (ii) $\rightarrow$ (iii). If no $\lambda_{j}=0$, let $u_{i j}$ be a standard set of matrix units for $M$ relative to the given standard diagonal expansion of $\Omega$. Then $u_{j i} \Omega=\lambda_{i}^{1 / 2} \psi_{1 j} \otimes \psi_{2 i}$. Since the standard diagonal expansion of $\Omega$ must contain a complete basis for at least one of $H_{1}, H_{2}$, and we have $\operatorname{dim} H_{1}=\operatorname{dim} H_{2}<\infty$, it follows that $M \Omega$ contains a basis for $H$. Thus (iii) $\rightarrow$ (i). By a similar argument $\Omega$ is cyclic for $M^{\prime}$, hence separating for $M$ and (iii) $\rightarrow$ (ii). Q.E.D.

It should be noted that if $\operatorname{dim} H_{1}=\operatorname{dim} H_{2}=\infty$, then the condition that $\Omega$ is cyclic for $M$ is not equivalent to the condition that $\Omega$ is separating for $M$. To see this, let $\Omega=\sum \lambda_{i}^{1 / 2} \psi_{\mu_{1}} \otimes \psi \psi_{r_{2 i}}$ where $\lambda_{i} \neq 0$ and $\psi_{1 i}$ is a complete basis for $H_{1}$, but the summation does not run over a complete basis for $H_{2}$. Then $\Omega$ is separating but not cyclic for $M$.

Corollary 2.11. Given $H=H_{1} \otimes H_{2}, \operatorname{dim} H_{1}=\operatorname{dim} H_{2}, \quad M=\mathscr{B}\left(H_{1}\right)$ $\otimes 1$. The set of all cyclic and separating unit vectors for $M$ is dense in the set of all unit vectors.

Proof. Given $\varepsilon>0, \psi \in H,\|\psi\|=1$. Let $\psi=\sum_{i=1}^{N} \lambda_{i}^{1 / 2} \psi_{1 i} \otimes \psi_{2 i}$ be a standard diagonal expansion of $\psi$ where $N=\operatorname{dim} H_{1}$. Choose $n<\infty$, $n \leq N$ so that $\sum_{i=n+1}^{N} \lambda_{i}<\varepsilon$, and let $\psi^{\prime}=\sum_{i=1}^{n} \lambda_{i}^{1 / 2} \psi_{1 i} \otimes \psi_{2 i}$. Then $\left\|\psi-\psi^{\prime}\right\|^{2}<\varepsilon$. Now choose orthonormal bases $\Phi_{k i}, i=1,2, \cdots$ for $H_{k}, k=1,2$ such that $\Phi_{k i}=\psi_{k i}, i=1, \cdots n$. Let $\psi^{\prime \prime}=\sum_{i=1}^{N} \lambda_{i}^{1 / 2} \Phi_{1 i} \otimes \Phi_{2 i}$, then $\left\|\psi^{\prime}-\psi^{\prime \prime}\right\|^{2}<\varepsilon$. Let 


$$
\Omega=\sum_{i=1}^{N} \mu_{i}^{1 / 2} \Phi_{1 i} \otimes \Phi_{2 i}
$$

where

$$
\mu_{i}=\left(\lambda_{i}+\varepsilon_{i}\right)(1+\varepsilon)^{-1},
$$

$\varepsilon_{i}>0, \sum \varepsilon_{i}=\varepsilon$. Then $\|\Omega\|=1$ and by the same argument used in the proof of lemma $2.10 \Omega$ is cyclic and separating for $M$. We have $\left\|\Omega-\psi^{\prime \prime}\right\|^{2}=\sum\left(\mu_{i}^{1 / 2}-\lambda_{i}^{1 / 2}\right)^{2}<2 \varepsilon$. Thus $\|\Omega-\psi\| \rightarrow 0$ as $\varepsilon \rightarrow 0 . \quad$ Q.E.D.

Let $R \subset \mathscr{B}(H)$ be a von Neumann algebra. Let $P=E E^{\prime}$ where $E, E^{\prime}$ are projections in $R, R^{\prime}$ respectively. Then

$$
R_{P}=\{P A P ; A \in R\}
$$

is a von Neumann algebra on $P H$. In particular if $R$ is a factor, $R_{E^{\prime}}$ is isomorphic to $R$. Thus by using projections in this way, one can either shrink or enlarge $R^{\prime}$ without changing the isomorphic class of $R$.

Lemma 2. 12. Given $\mathrm{R}\left(K_{\nu}, N_{\nu}, \psi_{\nu}\right)$ there exists $\mathrm{R}\left(H_{\nu}, M_{\nu}, \Omega_{\nu}\right) \sim$ $\mathrm{R}\left(K_{\nu}, N_{\nu}, \psi_{\nu}\right)$ such that $\Omega_{\nu}$ is cyclic and separating for $M_{\nu}$.

Proof. Write $K_{\nu}=K_{\nu 1} \otimes K_{\nu 2}$ where $N_{\nu}=\mathscr{B}\left(K_{\nu 1}\right) \otimes 1$. Let $n_{\nu}=$ $\operatorname{dim} K_{\nu 1}, J_{+}=\left\{\nu: \operatorname{dim} K_{\nu 2}>n_{\nu}\right\}$, and $J_{-}=\left\{\nu: \operatorname{dim} K_{\nu 2}<n_{\nu}\right\}$.

If $\nu \in J_{+}$let $\psi_{\nu}=\sum \lambda_{\nu j}^{1 / 2} \psi_{1 j}^{\nu} \otimes \psi_{r_{j}}^{\nu}$ be a standard diagonal expansion of $\psi_{\nu}$ relative to $N_{\nu}$. It follows that there is a projection $P_{\nu} \in N_{\nu}^{\prime}$ such that $P_{\nu} \psi_{\nu}=\psi_{\nu}$ and $\operatorname{dim} P_{\nu} K_{\nu}=n_{\nu}^{2}$. Define $H_{\nu}=P_{\nu} K_{\nu}, M_{\nu}=\left(N_{\nu}\right)_{P_{\nu}}$ and $\omega_{\nu}=\psi_{\nu}$. Since $P_{+}=\Pi\left(\pi P_{\nu}\right)$ is a projection in $\mathrm{R}\left(K_{\nu}, N_{\nu}, \psi_{\nu} ; \nu \in J_{+}\right)^{\prime}$ it follows that $\mathrm{R}\left(K_{\nu}, N_{\nu}, \psi_{\nu} ; \nu \in J_{+}\right)$and $\mathrm{R}\left(H_{\nu}, M_{\nu}, \omega_{\nu} ; \nu \in J_{+}\right)$are isomorphic.

If $\nu \in J_{-}$, imbed $K_{\nu 2}$ as a subspace of an $n_{\nu}$-dimensional space $H_{\nu 2}$. Define $H_{\nu}=K_{\nu 1} \otimes H_{\nu 2}, M_{\nu}=\mathscr{B}\left(K_{\nu 1}\right) \otimes 1, \omega_{\nu}=\psi_{\nu}$ (imbedded in $H_{\nu}$ ). Let $P_{\nu}$ be the projection onto $K_{\nu 1} \otimes K_{\nu 2}$. Then $P_{-}=\prod_{\nu \in J}\left(\pi P_{\nu}\right)$ is a projection in $\mathrm{R}\left(H_{\nu}, M_{\nu}, \omega_{\nu} ; \nu \in J_{-}\right)^{\prime}$ and it follows that $\mathrm{R}\left(H_{\nu}, M_{\nu}, \omega_{\nu} ; \nu \in J_{-}\right)$ is isomorphic to $\mathrm{R}\left(K_{\nu}, N_{\nu}, \psi_{\nu} ; \nu \in J_{-}\right)$. For $\nu \notin J_{+} \cup J_{-}$define $H_{\nu}=K_{\nu}$, $M_{\nu}=N_{\nu}, \omega_{\nu}=\psi_{\nu}$.

Thus we have $\mathrm{R}\left(H_{\nu}, M_{\nu}, \omega_{\nu} ; \nu \in J\right)$ isomorphic to $\mathrm{R}\left(K_{\nu}, N_{\nu}, \psi_{\nu}\right.$; $\nu \in J)$ where $H_{\nu}=H_{\nu 1} \otimes H_{\nu 2}{ }^{*} \quad M_{\nu}=\mathscr{B}\left(H_{\nu 1}\right) \otimes 1, \quad\left\|\omega_{\nu}\right\|=\left\|\psi_{\nu}\right\|, \operatorname{dim} H_{\nu 1}=$ 
$\operatorname{dim} H_{\nu 2}$ for all $\nu \in J$. It follows from corollary 2.11 that we can choose vectors $\Omega_{\nu} \in H_{\nu}$ which are cyclic and separating for $M_{\nu}$ such that $\left\|\omega_{\nu}-\Omega_{\nu}\right\|<2^{\nu}$. Hence $\otimes \Omega_{\nu}$ and $\otimes \omega_{\nu}$ are in the same strong equivalence class (see Eq. (2.6)) and $\mathrm{R}\left(H_{\nu}, M_{\nu}, \omega_{\nu}\right)=\mathrm{R}\left(H_{\nu}, M_{\nu}, \Omega_{\nu}\right)$.

Q.E.D.

Given $H_{\nu}=H_{\nu 1} \otimes H_{\nu 2}, M_{\nu}=\mathscr{B}\left(H_{\nu 1}\right) \otimes 1$, let $\Omega_{\nu 1}, \Omega_{\nu 2}$ be unit vectors in $H_{\nu}$. If there exist unitary operators $U_{\nu}=U_{\nu 1} \otimes U_{\nu 2}$ such that $U_{\nu} \Omega_{\nu 1}$ is in the strong equivalence class of $\Omega_{\nu 2}$ then $U R\left(M_{\nu}, \Omega_{\nu 1}\right) U^{-1}=$ $\mathrm{R}\left(M_{\nu}, \Omega_{\nu 2}\right)$ where $U=\otimes U_{\nu}$ (note that $\otimes U_{\nu}$ is not considered as an operator on the ITPS $\otimes\left(H_{\nu}, \Omega_{\nu 1}\right)$, but as an operator from $\otimes\left(H_{\nu}, \Omega_{\nu 1}\right)$ to $\left.\otimes\left(H_{\nu}, \Omega_{\nu 2}\right)\right)$. The following lemma states this condition in terms of the eigenvalue lists.

Lemma 2. 13. Given $H_{\nu}=H_{\nu 1} \otimes H_{\nu 2}, \quad M_{\nu}=B\left(H_{\nu 1}\right) \otimes 1$ and unit vectors $\Omega_{\nu 1}, \Omega_{\nu 2} \in H_{\nu}$. Let $\operatorname{Sp}\left(\Omega_{\nu_{i}} / M_{\nu}\right)=\left\{\lambda_{\nu j}^{i}\right\}, i=1,2$. If

$$
\sum_{\nu}\left[1-\sum_{j}\left(\lambda_{\nu j}^{1} \lambda_{\nu j}^{2}\right)^{1 / 2}\right]=\frac{1}{2} \sum_{\nu, j}\left(\left[\lambda_{\nu j}^{1}\right]^{1 / 2}-\left[\lambda_{\nu j}^{2}\right]^{1 / 2}\right)^{2}<\infty
$$

then $\mathrm{R}\left(M_{\nu}, \Omega_{\nu 1}\right)$ and $\mathrm{R}\left(M_{\nu}, \Omega_{\nu 2}\right)$ are unitarily equivalent.

Proof. Let $\psi_{1 j}^{\nu i}$ and $\psi_{2 j}^{\nu i}$ be orthogonal vectors corresponding to $\lambda_{\nu j}^{i}$ in a standard diagonal expansion of $\Omega_{\nu i}, i=1,2$. It is evidently possible to choose $\psi$ 's so that $\psi_{2 j}^{\nu 1}$ and $\psi_{2 j}^{\nu 2}$ are normalized or 0 simultaneously. We also supply, if necessary, additional indices so that $\psi_{2 j}^{v i}$ are complete. Define unitary operators

$$
\begin{aligned}
& U_{\nu k} \psi_{k j}^{\nu 1}=\psi_{k j}^{\nu 2}, \quad k=1,2 \\
& U_{\nu}=U_{\nu 1} \otimes U_{\nu 2} .
\end{aligned}
$$

Then $U_{\nu} \Omega_{\nu 1}$ is in the strong equivalence class of $\Omega_{\nu 2}$ if

$$
\infty>\Sigma_{\nu}\left|1-\left(\Omega_{\nu 2}, U_{\nu} \Omega_{\nu 1}^{1}\right)\right|=\sum_{\nu}\left|1-\sum_{j}\left(\lambda_{\nu j}^{1} \lambda_{\nu j}^{2}\right)^{1 / 2}\right| .
$$

The following lemma gives some known results which we shall have occasion to use

Lemma 2. 14. Given $M=\mathrm{R}\left(M_{\nu}, \Omega_{\nu}\right)$ where $M_{\nu}$ is type $I_{n_{\nu}}$, 
$2 \leq n_{\nu} \leq \infty$, and $\operatorname{Sp}\left(\Omega_{\nu} / M_{\nu}\right)=\left\{\lambda_{\nu_{i}}, i=1,2, \cdots n_{\nu}\right\}$

1) $M$ is type I if and only if

$$
\sum_{\nu \mid}\left|1-\lambda_{\nu 1}\right|<\infty
$$

2) $\quad M$ is type $\mathrm{II}_{1}$ if and only if $n_{\nu}<\infty$ for all $\nu$ and

$$
\sum_{\nu, i} i\left(n_{\nu}\right)^{-1 / 2}-\left.\left(\lambda_{\nu_{i}}\right)^{1 / 2}\right|^{2}<\infty
$$

3) If $\lambda_{\nu 1} \geq \delta$ for some $\delta>0$ for all $\nu$, then $M$ is type III if and only if

$$
\sum_{\nu, i} \lambda_{\nu i} \inf \left\{\left|\left(\lambda_{\nu 1} / \lambda_{\nu i}\right)-1\right|^{2}, C\right\}=\infty
$$

for some (and hence all) positive $C$.

Proof. For the type I conditions, see Araki [1] and Bures [6]. For the type $\mathrm{II}_{1}$ and III conditions, see Pukanszky [14], Bures [6], and Moore [10].

Q.E.D.

The type $\mathrm{I}$ and $\mathrm{II}_{1}$ conditions also follow from our results (see definition 8.2, lemmas $8.14,8.15,8.16$ and theorem 9.1).

\section{Asymptotic Ratio Set for ITPFI Factors}

In this section we define the asymptotic ratio set for ITPFI factors and give some of its properties.

Consider $\mathrm{R}\left(H_{\nu}, M_{\nu}, \Omega_{\nu} ; \nu \in A\right)$, and a finite subset $I \subset A$. Let $\operatorname{Sp}\left(\Omega_{\nu} / M_{\nu}\right)=\left\{\lambda_{\nu j}\right\}$, then any $\lambda \in \operatorname{Sp}(\Omega(I) / M(I))$ is of the form $\lambda=\prod_{\nu \in I} \lambda_{\nu, k(\nu)}$ for some function $k(\nu)$.

Definition 3. 1. Given $\mathrm{R}\left(H_{\nu}, M_{\nu}, \Omega_{\nu} ; \nu \in A\right)$ and a finite $I \subset A$, for any $K \subset \operatorname{Sp}(\Omega(I) / M(I))$ we define

$$
\lambda(K)=\sum_{\lambda \in K} \lambda .
$$

Definition 3.2. The asymptotic ratio set of $M=\mathrm{R}\left(M_{\nu}, \Omega_{\nu}\right)$, denoted by $\mathrm{r}_{\infty}(M, \Omega)$, is the set of all $x \in[0, \infty]$ such that there exists a sequence of mutually disjoint finite index sets $I_{n} \subset A, n \in I_{\infty}$, mutually disjoint subsets $K_{n}^{1}, K_{n}^{2}$ of $\operatorname{Sp}\left(\Omega\left(I_{n}\right) / M\left(I_{n}\right)\right)$ for each $n$ 
such that $\lambda \in K_{n}^{1}$ implies $\lambda \neq 0$, and a bijection $\phi_{n}$ from $K_{n}^{1}$ to $K_{n}^{2}$ satisfying

$$
\sum_{n} \lambda\left(K_{n}^{1}\right)=\infty
$$

and

$$
\lim _{n \rightarrow \infty} \max _{\lambda \in K_{n}^{1}}\left|x-\phi_{n} \lambda / \lambda\right|=0 \text {. }
$$

Such a sequence $\left(I_{n}, K_{n}^{i}, \phi_{n}\right)$ is called an $x$-sequence $\left(K_{n}^{1}\right.$ and $K_{n}^{2}$ are to be regarded disjoint even if they contain the same eigenvalue as long as the total number does not exceed the multiplicity of the eigenvalue).

As defined here, $\mathrm{r}_{\infty}(M, \Omega)$ could depend on the tensor product factorization $M=\otimes M_{\nu}$ as well as on $\Omega$. However, it will be shown that $\mathrm{r}_{\infty}(M, \Omega)$ is an algebraic invariant of $M$ (Theorem 5.9) and therefore depends neither on the vector $\Omega$ nor on the factorization. Since we do not need to indicate explicitly the possible dependence on the factorization in the following, we shall not do so.

It should be noted that in definition 3.2, $\Omega_{\nu}$ need not be a unit vector. Let $\operatorname{Sp}\left(\Omega_{\nu} / M_{\nu}\right)=\left(\lambda_{\nu 1}, \cdots \lambda_{\nu n_{\nu}}\right)$. Then $\sum_{i=1}^{n_{\nu}} \lambda_{\nu_{i}}=\left\|\Omega_{\nu}\right\|^{2} \neq 1$ in general. However it follows from $0<\Pi\left\|\Omega_{\nu}\right\|^{2}<\infty$ that $\sum_{i=1}^{n_{\nu}} \lambda_{\nu_{i}} \rightarrow 1$ sufficiently fast that

$$
\sum_{\nu}\left|1-\sum_{i=1}^{n_{\nu}} \lambda_{\nu_{i}}\right|<\infty
$$

Lemma 3. 3. Given $\varepsilon_{m}>0$ and $x \in \mathrm{r}_{\infty}(M, \Omega)$ there exists an $x$ sequence $\left(I_{m}, K_{m}^{i}, \phi_{m}\right)$ satisfying

$$
\left|1-\lambda\left(K_{m}^{1}\right)-\lambda\left(K_{m}^{2}\right)\right|<\varepsilon_{m} .
$$

Proof. Without loss of generality, we can assume $\lim \varepsilon_{m}=0$. Let $\left(J_{n}, L_{n}^{i}, \psi_{n}\right)$ be an $x$-sequence. Let

$$
Q_{n}=\left\|\Omega\left(J_{n}\right)\right\|^{2}
$$

Since

$$
0<\Pi Q_{n}<\infty
$$


we have

$$
\sum\left|1-Q_{n}\right|<\infty
$$

and Eq. (3.2) gives

$$
\sum\left|1-Q_{n}+\lambda\left(L_{n}^{1}\right)\right|=\infty
$$

Thus

$$
\prod_{n>N}\left[Q_{n}-\lambda\left(L_{n}^{1}\right)\right]=0
$$

for arbitrary $N$. It follows from Eqs. (3.5), (3.6) that we can inductively choose mutually disjoint finite index sets $A_{m}, m \in I_{\infty}$ such that

$$
\left|1-\prod_{n \in A_{m}} Q_{n}\right|<\varepsilon_{m} / 2
$$

and

$$
\prod_{n \in A_{m}}\left[Q_{n}-\lambda\left(L_{n}^{1}\right)\right]<\varepsilon_{m} / 2
$$

Define $I_{m}=\bigcup_{n \in A_{m}} J_{n}$. For each $\lambda \in \operatorname{Sp} \Omega\left(I_{m}\right)$ we have $\lambda=\prod_{n \in A_{m}} \lambda(n)$ where $\lambda(n) \in \operatorname{Sp} \Omega\left(J_{n}\right)$. Define $n(\lambda)$ as the smallest $n \in J_{m}$ such that $\lambda(n) \in L_{n}^{1} \cup L_{n}^{2}$ if such $n$ exists, otherwise define $n(\lambda)=\infty$. Define

$$
K_{m}^{i}=\left\{\lambda \in \operatorname{Sp} \Omega\left(I_{m}\right): n(\lambda) \neq \infty, \lambda(n(\lambda)) \in L_{n}^{i}, \lambda(n) \neq 0 \text { for } n \neq n(\lambda)\right\} .
$$

For $\lambda \in K_{m}^{1}$ define

$$
\left(\phi_{m} \lambda\right)(n)= \begin{cases}\lambda(n) & \text { if } n \neq n(\lambda), n \in A_{m} \\ \psi_{r_{n(\lambda)} \lambda(n(\lambda))} & \text { if } n=n(\lambda) .\end{cases}
$$

Using Eq. (3.3) we have

$$
\begin{aligned}
\lim _{m \rightarrow \infty} \max _{\lambda \in K_{m}^{1}} & \left|x-\phi_{m} \lambda / \lambda\right| \\
& =\lim _{m \rightarrow \infty} \max _{n \in A_{m}} \max _{\lambda(n) \in L_{n}^{1}}\left|x-\psi_{n} \lambda(n) / \lambda(n)\right|=0
\end{aligned}
$$

thus

$$
\lim _{m \rightarrow \infty} \max _{\lambda \in K_{m}^{1}}\left|x-\phi_{m} \lambda / \lambda\right|=0
$$

If $\lambda \in \operatorname{Sp} \Omega\left(I_{m}\right)$ then $\lambda \notin K_{m}^{1} \cup K_{m}^{2}$ only if $\lambda=0$ or $\lambda(n) \notin L_{n}^{1} \cup L_{n}^{2}$ for all $n \in A_{m}$. It follows that 


$$
\begin{aligned}
0 \leq & \prod_{n \in A_{m}} Q_{n}-\lambda\left(K_{m}^{1}\right)-\lambda\left(K_{m}^{2}\right) \\
& =\prod_{n \in A_{m}}\left[Q_{n}-\lambda\left(L_{n}^{1}\right)-\lambda\left(L_{n}^{2}\right)\right] \\
& \leq \prod_{n \in A_{m}}\left[Q_{n}-\lambda\left(L_{n}^{1}\right)\right]<\varepsilon_{m} / 2
\end{aligned}
$$

where we used Eq. (3.8). It follows from Eqs. (3.10), (3.7) that Eq. (3.4) is satisfied. It follows from Eq. (3.9) that

$$
\lim _{m \rightarrow \infty} \lambda\left(K_{m}^{2}\right) / \lambda\left(K_{m}^{1}\right)=x .
$$

Since $\lim \varepsilon_{n}=0$, Eq. (3.10) then implies that $\lim \lambda\left(K_{m}^{1}\right)=(1+x)^{-1}$. Thus $\sum \lambda\left(K_{m}^{1}\right)=\infty$ and $\left(I_{m}, K_{m}^{i}, \phi_{m}\right)$ is an $x$-sequence.

Q.E.D.

Corollary 3.4. If $x \in \mathrm{r}_{\infty}(M, \Omega)$ there exists an $x$-sequence $\left(I_{m}, K_{m}^{i}, \phi_{m}\right)$ satisfying

$$
\begin{aligned}
& \lim _{m \rightarrow \infty} \lambda\left(K_{m}^{1}\right)=(1+x)^{-1} \\
& \lim _{m \rightarrow \infty} \lambda\left(K_{m}^{2}\right)=x /(1+x) \\
& \quad=\left(1+x^{-1}\right)^{-1} \quad \text { if } x \neq 0 .
\end{aligned}
$$

Lemma 3. 5. Given $\mathrm{R}\left(H_{\nu}, M_{\nu}, \Omega_{\nu}\right), \operatorname{Sp}\left(\Omega_{\nu} / M_{\nu}\right)=\left\{\lambda_{\nu j}\right\}$. If there are subsequences $\nu(m), j_{1}(m), j_{2}(m)$ such that $\lambda_{\nu(m), j_{1}(m)} \rightarrow \lambda_{1} \neq 0$ and $\lambda_{\nu(m), j_{2}(m)} \rightarrow \lambda_{2}$, then $\lambda_{2} / \lambda_{1} \in \mathrm{r}_{\infty}(M, \Omega)$.

Proof. Let $I_{m}=\{\nu(m)\}, K_{m}^{i}=\left\{\lambda_{\nu(m), j_{i}(m)}\right\}, i=1,2$ and $\phi_{m} \lambda_{\nu(m), j_{1}(m)}=$ $\lambda_{\nu(m), j_{2}(m)}$. Clearly $\left(I_{m}, K_{m}^{i}, \phi_{m}\right)$ is a $\left(\lambda_{2} / \lambda_{1}\right)$-sequence. Q.E.D.

Lemma 3. 6. $\mathrm{r}_{\infty}(M, \Omega)-\{0\}$ is a multiplicative subgroup of $(0, \infty)$.

Proof. Let $x \in \mathrm{r}_{\infty}(M, \Omega)-\{0\}$. Choose an $x$-sequence $\left(I_{n}, K_{n}^{1}, K_{n}^{2}\right.$, $\left.\phi_{n}\right)$ as in Corollary 3.4. Then $\left(I_{n}, K_{n}^{2}, K_{n}^{1}, \phi_{n}^{-1}\right)$ is an $x^{-1}$-sequence.

Let $x, y \in \mathrm{r}_{\infty}(M, \Omega)-\{0\}$. Choose $x$ and $y$-sequences $\left(I_{n}, K_{n}^{x i}, \phi_{n}^{x}\right)$ and $\left(I_{n}^{y}, K_{n}^{y i}, \phi_{n}^{y}\right)$ as in Corollary 3.4. Choose subsequences $p_{n}, q_{n}, n \in$ $I_{\infty}$ such that $I_{p_{n}}^{x}$ and $I_{q_{n}}^{y}$ are mutually disjoint. Define $I_{n}=I_{p_{n}} \cup I_{q_{n}}$, $K_{n}^{i}=\left\{\lambda_{x} \lambda_{y}: \lambda_{x} \in K_{p_{n}}^{x i}, \lambda_{y} \in K_{q_{n}}^{y i}\right\}, \phi_{n}=\phi_{p_{n}}^{x} \phi_{q_{n}}^{y}$. Then

$$
\lim _{n \rightarrow \infty} \max _{\lambda \in K_{n}^{1}}\left|x y-\phi_{n} \lambda / \lambda\right|=0 \text {. }
$$


Also

$$
\lim _{n \rightarrow \infty} \lambda\left(K_{n}^{1}\right)=(1+x)^{-1}(1+y)^{-1}
$$

thus $\sum \lambda\left(K_{n}^{1}\right)=\infty$, and $\left(I_{n}, K_{n}^{i}, \phi_{n}\right)$ is an $x y$-sequence.

Q.E.D.

Lemma 3. 7. $\mathrm{r}_{\infty}(M, \Omega)$ is closed.

Proof. Let $x_{p} \in \mathrm{r}_{\infty}(M, \Omega), x=\lim x_{p}$. Without loss of generality we can assume $x_{p}<y$ for some $y<\infty$ and all $p$. It then follows from Corollary 3.4 that there exist $x_{p}$-sequences $\left(I_{n}^{p}, K_{n}^{p i}, \phi_{n}^{p}\right)$ such that

$$
\lambda\left(K_{n}^{p 1}\right)>\frac{1}{2}(1+y)^{-1}>0
$$

for all $p, n$. For each $p \in I_{\infty}$ choose $n_{p}$ inductively such that $I_{n_{p}}^{p}$ is disjoint from $I_{n_{q}}^{q}$ for $q<p$, and such that

$$
\lim _{p \rightarrow \infty} \max _{\lambda \in K_{n_{p}}^{p l}}\left|x_{p}-\phi_{n_{p}}^{p} \lambda / \lambda\right|=0 .
$$

It follows from Eq. (3.15) that $\sum_{p=1}^{\infty} \lambda\left(K_{n_{p}}^{p}\right)=\infty$, hence $\left(I_{n_{p}}^{p}, K_{n_{p}}^{p i}, \phi_{n_{p}}^{p}\right.$; $\left.p \in I_{\infty}\right)$ is an $x$-sequence.

Q.E.D.

Lemma 3. 8. Given $\mathrm{R}\left(M_{\nu}, \Omega_{\nu}\right)$ where $M_{\nu}$ is type $\mathrm{I}_{n_{\nu}}$, and $\operatorname{Sp}\left(\Omega_{\nu} / M_{\nu}\right)=\left\{\lambda_{\nu j}, j=1, \cdots n_{\nu}\right\}$. If $\sum\left|1-\lambda_{\nu 1}\right|<\infty$ then $\mathrm{r}_{\infty}(M, \Omega)=\{0\}$. If $\sum\left|1-\lambda_{\nu 1}\right|=\infty$ then $1 \in \mathrm{r}_{\infty}(M, \Omega)$.

Proof. We have $0<\Pi\left\|\Omega_{\nu}\right\|<\infty$ and thus $\sum_{j=1}^{n_{\nu}} \lambda_{\nu j}=\left\|\Omega_{\nu}\right\|_{1}=1+\delta_{\nu}$ where $\sum\left|\delta_{\nu}\right|<\infty$. By lemma 3.14 (which depends only on definition 3. 2) $\mathrm{r}_{\infty}(M)$ is unaffected by the change $\lambda_{\nu j} \rightarrow \lambda_{\nu j} /\left\|\Omega_{\nu}\right\|$. Since the condition $\sum\left|1-\lambda_{\nu 1}\right|<\infty$ is also unaffected by this change, we can assume $\sum \lambda_{\nu j}=1$ for all $\nu$.

If $\sum\left|1-\lambda_{\nu 1}\right|<\infty$ then $\lambda_{\nu 1} \rightarrow 1$, hence $\lambda_{\nu 2} \rightarrow 0$ and $0 \in \mathrm{r}_{\infty}(M, \Omega)$ by Lemma 3. 5. Let $\left(I_{m}, K_{m}^{i}, \phi_{m}\right)$ be an $x$-sequence for $(M, \Omega)$. Since $\lambda_{\nu 1} \neq 0$ and $\sum\left|1-\lambda_{\nu 1}\right|<\infty$ we have $\Pi \lambda_{\nu 1}>0$. Let $\lambda_{1}^{(m)}=\prod_{\nu \in I_{m}} \lambda_{\nu 1}$, then $\Pi_{m} \lambda_{1}^{(m)}>0$ which implies that $\sum_{m}\left(1-\lambda_{1}^{(m)}\right)<\infty$ and thus $\sum \lambda\left(K_{m}^{1}\right)=\infty$ if and only if $\lambda_{1}^{(m)} \in K_{m}^{1}$ for infinitely many $m$. Since 
$\Pi_{m} \lambda_{1}^{(m)}>0$ implies that $\lambda_{1}^{(m)} \rightarrow 1$, it follows that $x=0$, hence $\mathrm{r}_{\infty}(M, \Omega)=\{0\}$.

The second part of the lemma is more difficult, and we consider separately three different cases for $\left\{\lambda_{\nu j}\right\}$. Case (i), $\lambda_{\nu 2}$ has an accumulation point $\lambda_{2}>0$. Let $\lambda_{\nu(k), 2} \rightarrow \lambda_{2}$ as $k \rightarrow \infty$. Then $\lambda_{\nu(k), 1}$ must have an accumulation point $\lambda_{1} \geq \lambda_{2}$ and $\lambda_{2} / \lambda_{1} \in \mathrm{r}_{\infty}(M, \Omega)$ by lemma 3.5 . It then follows from lemma 3. 6 that $1 \in \mathrm{r}_{\infty}(M, \Omega)$.

Case (ii), $\lambda_{\nu 2} \rightarrow 0, \quad \lambda_{\nu 1} \rightarrow 1, \sum \lambda_{\nu 2}=\infty$. We can remove all $\nu$ with $\lambda_{\nu 2}=0$ and then reorder the remaining ones so that we have

$$
\lambda_{12} \geq \lambda_{22} \geq \cdots \geq 0
$$

which implies that

$$
\sum \lambda_{2 \nu, 2}=\infty
$$

For any $\varepsilon>0$ let

$$
I_{\varepsilon}=\left\{\nu: \lambda_{2 \nu+1,2} / \lambda_{2 \nu, 2}>1-\varepsilon\right\} .
$$

It follows from Eq. (3.17), (3.19) and the ratio test that

$$
\sum_{\nu \mp I_{\varepsilon}} \lambda_{2 \nu, 2}<\infty .
$$

Thus we can inductively choose mutually disjont finite sets $J_{n}, n \in I_{\infty}$ such that

$$
\sum_{\nu \in J_{n}} \lambda_{2 \nu, 2}>1
$$

and

$$
\left|1-\lambda_{2 \nu+1,2} / \lambda_{2 \nu, 2}\right|<\varepsilon_{n}
$$

for all $\nu \in J_{n}$, where $\varepsilon_{n} \rightarrow 0$. In this way we obtain a subsequence $\nu(j)$ such that

$$
\sum \lambda_{2 \nu(j), 2}=\infty
$$

and

$$
\lim _{j \rightarrow \infty} \lambda_{2 \nu(j)+1,2} / \lambda_{2 \nu(j), 2}=1
$$

Let $I_{j}=\{2 \nu(j), 2 \nu(j)+1\}, K_{j}^{1}=\left\{\lambda_{2 \nu(j), 2} \lambda_{2 \nu(j)+1,1}\right\}, K_{j}^{2}=\left\{\lambda_{2 \nu(j), 1} \lambda_{2 \nu(j)+1,2}\right\}$ and $\phi_{j}$ the unique bijection from $K_{j}^{1}$ to $K_{j}^{2}$. Since $\lambda_{\nu 1} \rightarrow 1$ it follows that 
$\sum \lambda\left(K_{j}^{1}\right)=\infty$ and $\left(I_{j}, K_{j}^{i}, \phi_{j}\right)$ is a 1 -sequence, and $1 \in \mathrm{r}_{\infty}(M, \Omega)$.

Case (iii), $\lambda_{\nu 2} \rightarrow 0$ and either $\sum \lambda_{\nu 2}<\infty$ or $\lambda_{\nu 1}$ has an accumulation point $\lambda_{1} \neq 1$. It follows in either case that

$$
\sum_{\nu} \sum_{j=3}^{n_{\nu}} \lambda_{\nu j}=\infty
$$

Let

$$
\begin{aligned}
& x_{\nu k}=\left(\begin{array}{ll}
\lambda_{\nu k} / \lambda_{\nu, k-1} & \text { if } \lambda_{\nu, k-1} \neq 0 \\
0 & \text { if } \lambda_{\nu, k-1}=0
\end{array}\right. \\
& P_{\nu}=\sum_{j=3}^{n_{\nu}}\left(\prod_{k=3}^{j} x_{\nu k}\right) \\
& \Lambda_{\nu}=\sum_{j=3}^{n_{\nu}} \lambda_{\nu j}=\lambda_{\nu 2} P_{\nu} .
\end{aligned}
$$

If $\sum \lambda_{\nu 2}<\infty$ it follows from Eqs. (3.25) and (3.28) that for all $N<\infty$ we have

$$
\sum_{P_{\nu}>N} \Lambda_{\nu}=\infty
$$

If $\lambda_{\nu 1}$ has an accumulation point $\lambda_{1} \neq 1$ then there is a subsequence $\nu(j)$ such that $\lambda_{\nu(j), 1} \rightarrow \lambda_{1}$, and hence $\Lambda_{\nu(j)} \rightarrow 1-\lambda_{1}$, and Eq. (3.29) holds. It follows from

$$
\sum_{m=0}^{\infty}(1-\varepsilon)^{m}=\varepsilon^{-1}, 0<\varepsilon<1
$$

that

$$
\sum_{x_{\nu}, 1-\varepsilon}\left(\prod_{k=3}^{j} x_{\nu k}\right)<\varepsilon^{-1}
$$

for a fixed $\nu$. Hence

$$
\sum_{x_{\nu j} \geqq 1-2 P_{\nu}^{-1}}\left(\prod_{k=3}^{j} x_{\nu_{k}}\right)>\frac{1}{2} P_{\nu}
$$

which implies that for each $\nu$ there are disjoint pairs $\left(\lambda_{\nu k}, \lambda_{\nu, k+1}\right)$ such that

$$
\lambda_{\nu, k+1} / \lambda_{\nu, k} \geqq 1-2 P_{\nu}^{-1}
$$

and

$$
\sum_{k} \lambda_{\nu k}>\frac{1}{4} P_{\nu} \lambda_{\nu 2}=\frac{1}{4} \Lambda_{\nu}
$$


Let $I_{\nu}=\{\nu\}$ and let $K_{\nu}^{1}, K_{\nu}^{2}$ contain the first and second members respectively of these disjoint pairs with $\phi_{\nu} \lambda_{\nu k}=\lambda_{\nu, k+1}$. It follows from Eqs. (3.29) and (3.34) that we can inductively choose mutually disjoint finite subsets $L_{n}$ such that

$$
\sum_{\nu \in L_{n}} \lambda\left(K_{\nu}^{1}\right)>1
$$

and

$$
1 \geqq \phi_{\nu} \lambda / \lambda>1-2 / n \text { if } \lambda \in K_{\nu}^{1}, \nu \in L_{n}
$$

which implies that $1 \in \mathrm{r}_{\infty}(M, \Omega)$.

Q.E.D.

Theorem 3.9. Let $\mathrm{R}\left(M_{\nu}, \Omega_{\nu}\right)$ be an ITPFI factor. Then $\mathrm{r}_{\infty}(M, \Omega)$ must be one of the following sets: $S_{x}, 0 \leq x \leq 1, S_{01}, S_{\infty}$.

Proof. The sets $S_{x}, 0 \leq x \leq 1, S_{01}, S_{\infty}$ are consistent with lemmas 3. 6 and 3. 7. By lemma 3.8. $\mathrm{r}_{\infty}(M, \Omega)$ is nonempty. If $\mathrm{r}_{\infty}(M, \Omega)$ is not one of the sets $S_{0}, S_{1}, S_{01}$ consider the set of all $l$ such that $e^{l} \in \mathrm{r}_{\infty}(M, \Omega)-\{0\}$. By lemma 3.6 this set must be of the form $\{n l, n=0, \pm 1, \cdots\}$ for some $0<l<\infty$, or be dense. In the former case we have $\mathrm{r}_{\infty}(M, \Omega)=S_{x}, x=e^{-l}$. If the latter case holds then $\mathrm{r}_{\infty}(M, \Omega)=[0, \infty)=S_{\infty}$ by lemma 3.7.

Q.E.D.

We now discuss some standard cases which have received some attention in the literature (von Neumann [12], Powers [13]). The following definition introduces our notation for these examples.

Definition 3.10. Let $M=\mathrm{R}\left(H_{\nu}, M_{\nu}, \Omega_{\nu}\right)$ be an ITPFI factor where $\operatorname{dim} H_{\nu}=4, M_{\nu}$ is type $\mathrm{I}_{2}$, and $\operatorname{Sp}\left(\Omega_{\nu} / M_{\nu}\right)=\left(\lambda_{1}, \lambda_{2}\right)$ independent of $\nu$. We denote the factor $M$ by $R_{x}$ where $x=\lambda_{2} / \lambda_{1}$.

Clearly $\mathrm{r}_{\infty}\left(R_{x}\right)=S_{x}$. By lemma $2.14, R_{0}$ is type $\mathrm{I}_{\infty}, R_{1}$ is hyperfinite $\mathrm{II}_{1}$, and $R_{x}, x \neq 0,1$ is type III. Powers [13] has shown that $R_{x}$ is nonisomorphic for different $x$. In the following we shall rederive this result in a more general context.

We now give some elementary properties of tensor products of ITPFI factors. More detailed results will be given later. Let $A_{1}, A_{2}$ be disjoint index sets and let $A=A_{1} \cup A_{2}$. Consider the ITPFI factors $M_{i}=\mathrm{R}\left(H_{\nu}, M_{\nu}, \Omega_{\nu} ; \nu \in A_{i}\right), i=1,2$. Then $M_{1} \otimes M_{2}=\mathrm{R}\left(H_{\nu}, M_{\nu}, \Omega_{\nu} ; \nu \in A\right)$. 
Clearly $x \in \mathrm{r}_{\infty}\left(M_{1}, \Omega\right)$ implies $x \in \mathrm{r}_{\infty}\left(M_{1} \otimes M_{2}, \Omega\right)$. Thus we obtain

Lemma 3. 11. Given $\mathrm{R}\left(M_{\nu}, \Omega_{\nu}\right), \mathrm{R}\left(N_{\alpha}, \psi_{\alpha}\right)$. Then

$$
\mathrm{r}_{\infty}(M \otimes N, \Omega \otimes \psi) \supset \mathrm{r}_{\infty}(M, \Omega) \cup \mathrm{r}_{\infty}(N, \psi) .
$$

If either $\mathrm{r}_{\infty}(M, \Omega)$ or $\mathrm{r}_{\infty}(N, \psi)$ is $S_{\infty}$, then

$$
\mathrm{r}_{\infty}(M \otimes N, \Omega \otimes \psi)=S_{\infty} .
$$

Definition 3.12. Given $0 \leq l_{1}, l_{2}<\infty$. If $l_{1} / l_{2}$ is rational, we define $\left(l_{1}, l_{2}\right)=l$ where $l$ is the largest number such that both $l_{1}$ and $l_{2}$ are integer multiples of $l$.

Lemma 3. 13. Given $0<x_{1}, x_{2} \leq 1, \mathrm{R}\left(M_{\nu}, \Omega_{\nu}\right), \mathrm{R}\left(N_{\alpha}, \psi_{\alpha}\right)$ and $\mathrm{r}_{\infty}(M, \Omega)=S_{x_{1}}, \mathrm{r}_{\infty}(N, \psi)=S_{x_{2}}$. Let $x_{1}=e^{-l_{1}}, x_{2}=e^{-l_{2}}$. If $l_{1} / l_{2}$ is irrational then

$$
\mathrm{r}_{\infty}(M \otimes N, \Omega \otimes \psi)=S_{\infty} .
$$

If $l_{1} / l_{2}$ is rational then

$$
\mathrm{r}_{\infty}(M \otimes N, \Omega \otimes \psi) \supset S_{x}
$$

where $x=e^{-\left(l_{1}, l_{2}\right)}$.

Proof. Follows from lemmas 3.6, 3.7, and 3.11. Q.E.D.

The following lemma proves that we can always take $\Omega_{\nu}$ to be a unit vector.

Lemma 3. 14. Given $\mathrm{R}\left(M_{\nu}, \Omega_{\nu}\right)$. Let $\Omega_{\nu}^{a}=\Omega_{\nu} /\left\|\Omega_{\nu}\right\|$. Then $\otimes \Omega_{\nu}^{a} \sim$ $\otimes \Omega_{\nu}$ and $\mathrm{r}_{\infty}\left(M, \Omega^{a}\right)=\mathrm{r}_{\infty}(M, \Omega)$.

Proof. Since

$$
0<\Pi\left\|\Omega_{\nu}\right\|<\infty
$$

we have

$$
\sum\left|1-\left(\Omega_{\nu}^{a}, \Omega_{\nu}\right)\right|=\sum\left|1-\left\|\Omega_{\nu}\right\|\right|<\infty
$$

and thus $\otimes \Omega_{\nu}^{a} \sim \otimes \Omega_{\nu}$. Let $\operatorname{Sp}\left(\Omega_{\nu} / M_{\nu}\right)=\left\{\lambda_{\nu j}\right\}$, then $\operatorname{Sp}\left(\Omega_{\nu}^{a} / M_{\nu}\right)=\left\{\alpha_{\nu j}\right\}$ where

$$
\alpha_{\nu j}=\lambda_{\nu j} /\left\|\Omega_{\nu}\right\|^{2}
$$

Let $\left(I_{n}, K_{n}^{i}, \phi_{n}\right)$ be an $x$-sequence for $\mathrm{R}\left(M_{\nu}, \Omega_{\nu}\right)$. The one-to-one map 
$\lambda_{\nu j} \rightarrow \alpha_{\nu j}$ defines a sequence $\left(I_{n}, K_{n}^{a i}, \phi_{n}^{a}\right)$. Eq. (3.38) gives

$$
\lim _{m \rightarrow \infty} \prod_{\nu>m}\left\|\Omega_{\nu}\right\|^{2}=1
$$

and thus $\sum \lambda\left(K_{n}^{1}\right)=\infty$ implies that

$$
\sum \lambda\left(K_{n}^{a l}\right)=\infty
$$

Eqs. (3.40), (3.41) imply that

$$
\phi_{n} \mu / \mu=\phi_{n}^{a} \mu^{a} / \mu^{a}
$$

where $\mu \rightarrow \mu^{a}, \phi_{n} \mu \rightarrow \phi_{n}^{a} \mu^{a}$. Thus $\left(I_{n}, K_{n}^{a i}, \phi_{n}^{a}\right)$ is an $x$-sequence for $\mathrm{R}\left(M_{\nu}, \Omega_{\nu}^{a}\right)$. Since the argument is reversible, $\mathrm{r}_{\infty}(M, \Omega)=\mathrm{r}_{\infty}\left(M, \Omega^{a}\right)$.

Q.E.D.

Lemma 3. 15. Given $\mathrm{R}\left(N_{\nu}, \psi_{\nu}\right)$ there exists $\mathrm{R}\left(M_{\nu}, \Omega_{\nu}\right) \sim \mathrm{R}\left(N_{\nu}\right.$, $\left.\psi_{\nu}\right)$ such that $\Omega_{\nu}$ is cyclic and separating for $M_{\nu}$ and $\mathrm{r}_{\infty}(M, \Omega)=$ $\mathrm{r}_{\infty}(N, \psi)$.

Proof. Construct $M_{\nu}, \omega_{\nu}$ as in lemma 2.12. Since $\operatorname{Sp}\left(\omega_{\nu} / M_{\nu}\right)=$ $\operatorname{Sp}\left(\psi_{\nu} / N_{\nu}\right)$ we have $\mathrm{r}_{\infty}(M, \omega)=\mathrm{r}_{\infty}(N, \psi)$. Let $\operatorname{Sp}\left(\omega_{\nu} / M_{\nu}\right)=\left\{\lambda_{\nu i}^{a}\right\}$ and let $m_{\nu}$ be the number of $\lambda_{\nu i}^{a}=0$. If $m_{\nu}=0$ let

$$
\lambda_{\nu i}^{b}=\lambda_{\nu i}^{a}
$$

If $m_{\nu} \neq 0$ let

$$
\lambda_{\nu i}^{b}= \begin{cases}\left(1-\varepsilon_{\nu}\right) \lambda_{\nu i}^{a} & \text { if } \lambda_{\nu i}^{a} \neq 0 \\ \varepsilon_{\nu} / m_{\nu} & \text { if } \lambda_{\nu i}^{a}=0\end{cases}
$$

where

$$
\varepsilon_{\nu}=2^{-\nu} \min _{k}\left\{\lambda_{\nu k}^{a} ; \lambda_{\nu k}^{a} \neq 0\right\}
$$

Now choose $\Omega_{\nu}$ as in lemma 2. 12 where $\operatorname{Sp}\left(\Omega_{\nu} / M_{\nu}\right)=\left\{\lambda_{\nu i}^{b}\right\}$. Let $\left(I_{n}, K_{n}^{a i}, \phi_{n}^{a}\right)$ be an $x$-sequence for $\mathrm{R}\left(M_{\nu}, \omega_{\nu}\right)$. The one-to-one map $\lambda_{\nu j}^{a} \rightarrow \lambda_{\nu j}^{b}$ defines a sequence $\left(I_{n}, K_{n}^{b i}, \phi_{n}^{b}\right)$. It follows from Eq. (3.46) that

$$
\lim _{m \rightarrow \infty} \prod_{\nu>m}\left(1-\varepsilon_{\nu}\right)=1
$$

and thus $\sum \lambda\left(K_{n}^{a 1}\right)=\infty$ implies that

$$
\sum \lambda\left(K_{n}^{b 1}\right)=\infty
$$


Since $\mu^{a} \in K_{n}^{a 1}$ implies $\mu^{a} \neq 0$ it follows from Eqs. (3. 44-47) that

$$
\lim _{n \rightarrow \infty} \max _{\mu^{a} \in K_{u}^{a}}\left|\phi_{n}^{a} \mu^{a} / \mu^{a}-\phi_{n}^{b} \mu^{b} / \mu^{b}\right|=0
$$

where $\mu^{a} \rightarrow \mu^{b}, \phi_{n}^{a} \mu^{a} \rightarrow \phi_{n}^{b} \mu^{b}$. It follows that $\left(I_{n}, K_{n}^{b i}, \phi_{n}^{b}\right)$ is an $x$-sequence for $\mathrm{R}\left(M_{\nu}, \Omega_{\nu}\right)$. Conversely let $\left(I_{n}, K_{n}^{b i}, \phi_{n}^{b}\right)$ be an $x$-sequence for $\mathrm{R}\left(M_{\nu}, \Omega_{\nu}\right)$. Let $K$ be the set of all $\mu^{b} \in K_{n}^{b 1}, n=1,2, \cdots$ which contain $\lambda_{\nu j}^{b}$ as a factor where $\lambda_{\nu j}^{a}=0$. It follows from Eqs. (3. 45-46) that

$$
\sum_{\mu, b K} \mu^{b}<\infty
$$

and thus we can remove all $\mu^{b} \in K$ from $K_{n}^{b 1}, n \in I_{\infty}$. The above argument can then be reversed and the sequence $\left(I_{n}, K_{n}^{a i}, \phi_{n}^{a}\right)$ defined by $\lambda_{\nu j}^{b} \rightarrow \lambda_{\nu j}^{a}$ is an $x$-sequence for $\mathrm{R}\left(M_{\nu}, \omega_{\nu}\right)$.

Q.E.D.

\section{Basic Technical Lemma}

In this section we prove a basic technical lemma which is concerned with the following situation. Let $M$ be a type $I_{m}$ factor on a Hilbert space $H$ and let $\Omega$ be a vector in $H$. Let $\left(\lambda_{1}, \cdots \lambda_{n}\right)$ be a possible eigenvalue list, that is $\lambda_{1} \geq \lambda_{2} \geq \cdots \geq \lambda_{n} \geq 0$. Let $\left(\mu_{1}, \cdots \mu_{l}\right)$ be another possible eigenvalue list. Suppose the list of products $\left\{\lambda_{i} \mu_{j}\right\}$ approximates in some suitable way the eigenvalue list $\operatorname{Sp}(\Omega / M)$. Then it should be possible to find a type $\mathrm{I}_{n}$ factor $M_{1}$ such that $(M, \Omega)$ is in some sense approximated by $\left(M_{1} \otimes M_{2}, \Phi_{1} \otimes \Phi_{2}\right)$ where $\operatorname{Sp}\left(\Phi_{1} / M_{1}\right)=\left(\lambda_{1}, \cdots \lambda_{n}\right)$.

Definition 4.1. Let $M$ be a factor on a Hilbert space $H, N$ a type I factor, $N \subset M$. We say that $\Phi \in H$ factorizes $N$ in $M$ if

$$
\begin{aligned}
& H=H_{1} \otimes H_{2} \\
& H_{1}=H_{11} \otimes H_{12} \\
& \Phi=\Phi_{1} \otimes \Phi_{2}, \Phi_{i} \in H_{i} \\
& N=\widehat{N} \otimes \mathbf{1} \\
& \widehat{N}=\mathscr{B}\left(H_{11}\right) \otimes \mathbf{1} \\
& M=\widehat{N} \otimes M_{2} .
\end{aligned}
$$


Lemma 4.2. Given $0<\varepsilon<1$, a Hilbert space $H$, a type I factor $M \subset \mathscr{B}(H)$, a unit vector $\Omega \in H,\left(\lambda_{1}, \cdots \lambda_{n}\right)$ satisfying $\lambda_{1} \geq \lambda_{2} \geq \cdots \geq \lambda_{n} \geq 0$ and $\sum \lambda_{j}=1$. Let $K_{1}, \cdots K_{n}$ be disjoint subsets of $\operatorname{Sp}(\Omega / M)$ such that each $K_{j}$ contains $k$ elements and $\lambda \in K_{1}$ implies $\lambda \neq 0$. Let $\Phi_{j}$ be a bijection from $K_{1}$ to $K_{i}, j=2, \cdots n$. Let $L=\operatorname{Sp}(\Omega / M)-\bigcup_{j=1}^{n} K_{j}$. Let $\varepsilon^{\prime}=\min \left(\varepsilon, \lambda_{j} / \lambda_{1}\right.$ for $\left.\lambda_{j} \neq 0\right)$. If

$$
\max _{j=2}^{n} \max _{\lambda \in K_{1}}\left|\left(\lambda_{j} / \lambda_{1}\right)^{1 / 2}-\left(\phi_{j} \lambda / \lambda\right)^{1 / 2}\right|<\varepsilon^{\prime}
$$

and

$$
\lambda(L)<\varepsilon
$$

then the following situation holds. There exist projections $P \in M$, $P^{\prime} \in M^{\prime}$, a type $\mathrm{I}_{n}$ factor $N \subset M_{P P^{\prime}}$, and a unit vector $\Phi \in P P^{\prime} H$ such that

$$
\begin{aligned}
& \left\|\left(1-P P^{\prime}\right) \Omega\right\|^{2}<n \varepsilon \\
& \left\|P P^{\prime} \Omega-\Phi\right\|<c_{n} \varepsilon
\end{aligned}
$$

where $c_{n}$ depends only on $n, \Phi$ factorizes $N$ in $M_{P P^{\prime}}$ and $\operatorname{Sp}(\Phi / N)$ $=\left\{\lambda_{1}, \cdots \lambda_{n}\right\}$.

Proof. Let $\Omega=\sum \omega_{\alpha}^{1 / 2} \psi_{1 \alpha} \otimes \psi_{2 \alpha}$ be a standard diagonal expansion of $\Omega$ (relative to $M$ ). We reindex the $\omega_{\alpha} \in \bigcup_{j=1}^{n} K_{j}$ as follows. Order the elements of $K_{1}$ by $\omega_{11} \geq \omega_{12} \geq \cdots \geq \omega_{1 k}$, and let $\omega_{j l}=\phi_{j} \omega_{1 l}, j=2, \cdots n$. Let $u_{\alpha \beta}, v_{\alpha \beta}$ be the standard matrix units for $M, M^{\prime}$ associated with this expansion. Define

$$
P=\sum_{i=1}^{n} \sum_{j=1}^{k} u_{i j, i j}
$$

If $\lambda_{n}=0$ choose $p$ so that $\lambda_{P} \neq 0, \lambda_{p+1}=0$, otherwise choose $p=n$. If $1 \leq i \leq p$ then Eq. (4.1) implies that $\omega_{i j} \neq 0$ since by assumption $\omega_{1 j} \neq 0$. Hence $v_{i j, i^{\prime} j^{\prime}} \neq 0$ for $i, i^{\prime}=1 \cdots p$. Since $\left\{\omega_{j l}\right\}$ is a subset of $\operatorname{Sp}(\Omega / M), u_{i j, i^{\prime} j^{\prime}} \neq 0$ for $i, i^{\prime}=1 \cdots n$. (See definition 2.3.) Define

$$
P^{\prime}=\sum_{i=1}^{p} \sum_{j=1}^{k} v_{i j, i j}
$$

Then $u_{i j, i^{\prime} j^{\prime}}, i, i^{\prime}=1, \cdots n$ and $v_{i j, i^{\prime} j^{\prime}}, i, i^{\prime}=1, \cdots p$ are matrix units for 
$M_{P P^{\prime}}$ and $M_{P P^{\prime}}^{\prime}$ respectively in $P P^{\prime} H$. Define matrix units $e_{i j}^{\nu,}, \nu$, $q=1,2$ by

$$
\begin{array}{ll}
e_{i j}^{11}=\sum_{l=1}^{k} u_{i l, j l} & e_{i j}^{12}=\sum_{l=1}^{k} v_{i l, j l} \\
e_{i j}^{21}=\sum_{l=1}^{n} u_{l i, l j} & e_{i j}^{22}=\sum_{l=1}^{p} v_{l i, l j} .
\end{array}
$$

One can easily verify that these four families of matrix units commute and that they are irreducible on $P P^{\prime} H$. Thus we obtain

$$
P P^{\prime} H=\bigotimes_{\nu=1}^{2}\left(H_{\nu 1} \otimes H_{\nu 2}\right)
$$

where $e_{i j}^{\nu q}$ are matrix units for $\mathscr{B}\left(H_{\nu q}\right) \otimes 1$. Thus $\operatorname{dim} H_{11}=n$, $\operatorname{dim} H_{12}=p, \operatorname{dim} H_{21}=\operatorname{dim} H_{22}=k$. We have

$$
P P^{\prime} \Omega=\sum_{i=1}^{p} \sum_{j=1}^{k} \omega_{i j}^{1 / 2} \psi_{1, i j} \otimes \psi_{2, i j}
$$

where $\psi_{q, i j} \in H_{1 q} \otimes H_{2 q}, q=1,2$. Thus

$$
\left\|\left(1-P P^{\prime}\right) \Omega\right\|^{2}=\omega(L)+\sum_{i=p+1}^{n} \sum_{j=1}^{k} \omega_{i j} .
$$

For $i>p$ Eq. (4.1) gives $\omega_{i j}<\left(\varepsilon^{\prime}\right)^{2} \omega_{1 j}<\varepsilon^{\prime} \omega_{1 j}$ since $\varepsilon^{\prime} \leq \varepsilon<1$. Since $p \geq 1$ and $\sum \omega_{1 j} \leq 1$ we get

$$
\left\|\left(1-P P^{\prime}\right) \Omega\right\|^{2}<\varepsilon+(n-1) \varepsilon^{\prime} \leq n \varepsilon .
$$

Now choose orthonormal bases $\psi_{i}^{\nu q}$ for $H_{\nu q}$ such that $\psi_{q, i j}=\psi_{i}^{1 q} \otimes \psi_{j}^{2 q}$. Define

$$
\begin{gathered}
\Phi_{1}=\sum_{i=1}^{p} \lambda_{i}^{1 / 2} \psi_{i}^{11} \otimes \psi_{i}^{12} \\
\mathscr{\Phi}_{2}^{\prime}=\sum_{j=1}^{k}\left(\omega_{1 j} / \lambda_{1}\right)^{1 / 2} \psi_{j}^{21} \otimes \psi_{j}^{22} \\
\Phi_{2}=\Phi_{2}^{\prime} /\left\|\Phi_{2}^{\prime}\right\| .
\end{gathered}
$$

We have

$$
\left\|P P^{\prime} \Omega-\sigma_{1} \otimes \Phi_{2}^{\prime}\right\|^{2}=\sum_{i=1}^{p} \sum_{j=1}^{k}\left(\omega_{i j}^{1 / 2}-\left(\lambda_{i} \omega_{1 j} / \lambda_{1}\right)^{1 / 2}\right)^{2}
$$

Eq. (4.1) gives

$$
\left|\omega_{i j}^{1 / 2}-\left(\lambda_{i} \omega_{1 j} / \lambda_{1}\right)^{1 / 2}\right|<\varepsilon^{\prime} \omega_{1 j}^{1 / 2}
$$

hence 


$$
\left\|P P^{\prime} \Omega-\Phi_{1} \otimes \Phi_{2}^{\prime}\right\|^{2}<p\left(\varepsilon^{\prime}\right)^{2} \leq n \varepsilon^{2}
$$

Since $\left\|\Phi_{1}\right\|^{2}=\sum \lambda_{i}=1$, it follows from Eqs. (4.13), (4.14) that

$$
\left\|\Phi_{1} \otimes \Phi_{2}-\Phi_{1} \otimes \Phi_{2}^{\prime}\right\|=\left\|\Phi_{2}-\Phi_{2}^{\prime}\right\|=\left|1-\left\{\sum_{j=1}^{k}\left(\omega_{1 j} / \lambda_{1}\right)\right\}^{1 / 2}\right| \text {. }
$$

Eq. (4.15) gives

$$
\left|\sum_{i=1}^{n} \sum_{j=1}^{k} \omega_{i j}-\sum_{i=1}^{n} \lambda_{i} \sum_{j=1}^{k}\left(\omega_{1 j} / \lambda_{1}\right)\right|<2 n \varepsilon^{\prime} \leq 2 n \varepsilon
$$

where we used $\sum \omega_{1 j} \leq 1, \sum_{j} \omega_{i j} \leq 1$ and $\varepsilon^{\prime} \leq \varepsilon<1$. Since $\sum \lambda_{i}=1$ and $\left|1-\sum \omega_{i j}\right|<\varepsilon$ we get

$$
\left|1-\sum\left(\omega_{1 j} / \lambda_{1}\right)\right|<(2 n+1) \varepsilon
$$

Eqs. (4.16), (4.17), (4.19) now give

$$
\left\|P P^{\prime} \Omega-\Phi_{1} \otimes \Phi_{2}\right\|<c_{n} \varepsilon
$$

where

$$
c_{n}=n^{1 / 2}+2 n+1
$$

Q.E.D.

We remark that if the dimensions of $M, M^{\prime}$ are consistent with setting $P=P^{\prime}=1$ in lemma 4.2 , then it is possible to choose $P=P^{\prime}=1$ in lemma 4.2. However we shall not make any use of this fact.

\section{Algebraic Invariance of $\mathbf{r}_{\infty}(\boldsymbol{M})$}

In this section we prove that $\mathrm{r}_{\infty}(M, \Omega)$ given by definition 3.2 for ITPFI factors $M=\mathrm{R}\left(M_{\nu}, \Omega_{\nu}\right)$ is an algebraic invariant.

We note that given an ITPFI factor $\mathrm{R}\left(M_{\nu}, \Omega_{\nu}\right)$, by lemma 3.14 we can assume $\left\|\Omega_{\nu}\right\|=1$. Unless stated explicitly to the contrary, we shall always take $\left\|\Omega_{\nu}\right\|=1$ in this section.

Lemma 5. 1. Given $M=\mathrm{R}\left(M_{\nu}, \Omega_{\nu} ; \nu \in A\right), \quad x \in r_{\infty}(M, \Omega), \quad x \leq 1$ and $0<\varepsilon<1$. Then there is a finite subset $I \subset A$, projections $P, P^{\prime}$ in $M(I), M(I)^{\prime}$ respectively, a unit vector $\Phi \in P P^{\prime} H(I)$, and a type $\mathrm{I}_{2}$ factor $N \subset M(I)_{P P^{\prime}}$ such that 


$$
\begin{aligned}
& \left\|\left(P P^{\prime}-1\right) \Omega(I)\right\|<\varepsilon \\
& \left\|P P^{\prime} \Omega(I)-\Phi\right\|<\varepsilon \\
& \operatorname{Sp}(\Phi / N)=(\lambda, 1-\lambda)
\end{aligned}
$$

where $x=(1-\lambda) / \lambda$, and $\Phi$ factorizes $N$ in $M(I)_{P P^{\prime}}$.

Proof. If $x=0$ choose $\varepsilon^{\prime}=\varepsilon, \varepsilon^{\prime \prime}=\varepsilon^{2}$. If $x \neq 0$ choose $\varepsilon^{\prime}=\min (\varepsilon, x)$, $\varepsilon^{\prime \prime}=x^{1 / 2} \varepsilon^{\prime}$. By Lemma 3.3 there exists $\left(I, K^{1}, K^{2}, \phi\right)$ such that

$$
\max _{\mu \in K^{1}}|x-\phi \mu / \mu|<\varepsilon^{\prime \prime}
$$

and

$$
\left|1-\lambda\left(K^{1}\right)-\lambda\left(K^{2}\right)\right|<\varepsilon / c_{2}
$$

where $c_{2}$ is given by lemma 4.2. Eq. (5.4) implies that

$$
\max _{\mu \in K 1}\left|x^{1 / 2}-(\phi \mu / \mu)^{1 / 2}\right|<\varepsilon^{\prime} \text {. }
$$

The result now follows from lemma 4.2.

Q.E.D.

Lemma 5.2. Let $M$ be a factor, $P$ a projection in $M$. If $M$ is infinite then $M \stackrel{u}{\sim} M_{P} \otimes I_{\infty}$. If $P$ is infinite then $M \stackrel{u}{\sim} M_{P}$.

Proof. Two projections $E, F \in M$ are equivalent (with respect to $M), E \sim F$, if there is a partial isometry $U \in M$ such that $U^{*} U=E$, $U U^{*}=F \quad$ (Murray and von Neumann [11]), which implies that $U M_{E} U^{*}=M_{F}$. Thus if $P$ is infinite then $P \sim 1$ and $M_{P} \sim M$. If $M$ is infinite, then for any $P \in M$ there exists a family of projections $P_{i} \in M, \quad i \in I_{\infty}$ such that $\sum P_{i}=1, P_{i} P_{j}=0$ for all $i \neq j$, and $P_{i} \sim P$ for all $i$. Let $M_{i j}=\left\{P_{i} A P_{j} ; A \in M\right\}=\left\{U_{i j} B ; B \in M_{P,}\right\}$ where $U_{i j} \in M$, $U_{i j}^{*}=U_{j i}, \quad U_{i j} U_{k l}=\delta_{j k} U_{i l}, \quad U_{i i}=P_{i}$. Then $M=\otimes M_{i j}$ which is unitarily equivalent to $M_{P} \otimes I_{\infty}$.

Q.E.D.

Definition 5.3. Given $M=\mathrm{R}\left(M_{\nu}, \Omega_{\nu}\right)$ where $\operatorname{Sp}\left(\Omega_{\nu} / M_{\nu}\right)=\left\{\lambda_{\nu j}\right\}$. We define

$$
\hat{\mathrm{r}}(M, \Omega)=\left\{\lambda_{\nu i} / \lambda_{\nu j} ; \lambda_{\nu j} \neq 0\right\} \text {. }
$$

Lemma 5. 4. Given $M=\mathrm{R}\left(H_{\nu}, M_{\nu}, \Omega_{\nu} ; \nu \in A\right), \quad N=\mathrm{R}\left(N_{\alpha}, \psi_{\alpha}\right)$ where all $N_{\alpha}$ are type $\mathrm{I}_{2}$. If $\hat{\mathrm{r}}\left(N, \psi_{r}\right) \subset \mathrm{r}_{\infty}(M, \Omega)$ then $M \sim M \otimes N$. 
Proof. We shall use lemma 5.1 to construct a projection $P \in M$ such that $M_{P}$ is an ITPFI factor and $M_{P} \sim M_{a} \otimes(\otimes N)^{\infty}$ where $(\otimes N)^{\infty}$ means the tensor product of $N$ with itself infinitely many times. Let $\mathrm{Sp}\left(\psi_{\alpha} / N_{\alpha}\right)=\left(\lambda_{\alpha}, 1-\lambda_{\alpha}\right), \quad x_{\alpha}=\left(1-\lambda_{\alpha}\right) / \lambda_{\alpha} \leq 1$. By corollary 3. 4 there exist $\quad x_{\alpha}$-sequences $(\alpha, n)=\left(I_{n}^{\alpha}, K_{n}^{\alpha i}, \phi_{n}^{\alpha}\right)$ for $M=\otimes M_{\nu}$ such that $\lambda\left(K_{n}^{\alpha i}\right)>1 / 3$ for all $(\alpha, n)$. Let $l(\alpha, k)$ be a one-to-one map of the pairs of positive integers $(\alpha, k)$ onto the positive integers. In the order of increasing $l(\alpha, k)$ we inductively select a subsequence $n(\alpha, k)$, $\alpha, k \in I_{\infty}$ such that $I_{n(\alpha, k)}^{\alpha} \cap I_{n\left(\alpha^{\prime}, k^{\prime}\right)}^{\alpha \prime}=\phi$ for all $\left(\alpha^{\prime}, k^{\prime}\right)$ with $l\left(\alpha^{\prime}, k^{\prime}\right)<$ $l(\alpha, k)$. Now reorder the sequence $k=1,2, \cdots$ into a double sequence $k=l(i, j), i, j \in I_{\infty}$ where $l(i, j)$ was introduced above. Let $M_{\alpha i}$ $=\bigotimes_{j=1}^{\infty} M\left(I_{i j}^{\alpha}\right), \quad \Omega_{\alpha i}=\bigotimes_{j=1}^{\infty} \Omega\left(I_{i j}^{\alpha}\right) \quad$ where $\quad I_{i j}^{\alpha}=I_{n(\alpha, l(i, j))}^{\alpha}$. By construction $x_{\alpha} \in \mathrm{r}_{\infty}\left(M\left(I_{i j}^{\alpha}\right), \Omega\left(I_{i j}^{\alpha}\right) ; j \in I_{\infty}\right)$ for each $\alpha$ and $i$. Now choose $\varepsilon_{\alpha i}>0$, $\sum \varepsilon_{\alpha i}<\infty$. For each $(\alpha, i)$, it follows from lemma 5.1 that there is a set $I_{\alpha i}$ which is a finite union of the $I_{i j}^{\alpha}, j \in I_{\infty}$, projections $P_{\alpha i} \in M\left(I_{\alpha i}\right), P_{\alpha i}^{\prime} \in M\left(I_{\alpha i}\right)^{\prime}$, a unit vector $\Phi_{\alpha i} \in P_{\alpha i} P_{\alpha i}^{\prime} H\left(I_{\alpha i}\right)$ such that

$$
\begin{aligned}
& \left\|\Omega_{\alpha i}-P_{\alpha i} P_{\alpha i}^{\prime} \Omega_{\alpha i}\right\|<\varepsilon_{\alpha i} \\
& \left|1-\left(\varpi_{\alpha i}, \widehat{\Omega}_{\alpha_{i}}\right)\right|<\varepsilon_{\alpha i}
\end{aligned}
$$

where

$$
\widehat{\Omega}_{\alpha_{i}}=P_{\alpha_{i}} P_{\alpha i}^{\prime} \Omega_{\alpha_{i}} /\left\|P_{\alpha i} P_{\alpha i}^{\prime} \Omega_{\alpha i}\right\| .
$$

Also there is a type $\mathrm{I}_{2}$ factor $N_{\alpha_{i}} \subset\left(M_{\alpha_{i}}\right)_{P_{\alpha i} P_{\alpha i}^{\prime}}$, such that $\Phi_{\alpha i}$ factorizes $N_{\alpha i}$ in $\left(M_{\alpha_{i}}\right)_{P_{\alpha_{i}} P_{\alpha i}^{\prime}}$, and $\operatorname{Sp}\left(\Phi_{\alpha_{i}} / N_{\alpha_{i}}\right)=\left(\lambda_{\alpha}, 1-\lambda_{\alpha}\right)$. Thus $\left(M_{\alpha_{i}}\right)_{P_{\alpha_{i} P_{\alpha}^{\prime} i}}=$ $M_{\alpha i}^{b} \otimes N_{\alpha i}$ where $\Phi_{\alpha i}=\Phi_{\alpha i}^{b} \otimes \Phi_{\alpha i}^{N}$. Let $P=\Pi P_{\alpha i}, P^{\prime}=\Pi P_{\alpha i}^{\prime}$. It follows from Eq. (5.7) that $P, P^{\prime} \neq 0$. Let $K=A-\cup I_{\alpha i}$. Since $\Phi_{\alpha i}$ is in the strong equivalence class of $\hat{\Omega}_{\alpha i}$ we have

$$
\begin{aligned}
M_{P P^{\prime}} & \left.=M(K) \otimes\left(\otimes M_{\alpha i}\right)_{P \alpha_{1} P_{\alpha i}^{\prime}}\right) \\
& =M(K) \otimes\left(\otimes M_{\alpha i}^{b}\right) \otimes\left\{\bigotimes_{i=1}^{\infty}\left(\bigotimes_{\alpha=1}^{\infty}\left(N_{\alpha i}, \Phi_{\alpha i}^{N}\right)\right)\right\} .
\end{aligned}
$$

By construction we have $\bigotimes_{\alpha=1}^{\infty}\left(N_{\alpha i}, \Phi_{\alpha i}^{N}\right) \sim N$ for each $i$ and hence $\bigotimes_{i}\left(\otimes_{\alpha} N_{\alpha i}\right) \sim(\otimes N)^{\infty} \sim \bigotimes_{i}\left(\bigotimes_{\alpha} N_{\alpha_{i}}\right) \otimes N$. Since $M_{\Gamma P^{\prime}} \sim M_{P} \quad$ we have $M_{P} \sim M_{P} \otimes N$. If $M$ is infinite, then by lemma 5.2 $M \sim M_{P} \otimes I_{\infty}$ and 
thus $M \sim M \otimes N$. If $M$ is hyperfinite $\mathrm{II}_{1}$ then $M_{P}$ is also hyperfinite $\mathrm{II}_{1}$, and $M \sim M_{P}$ hence $M \sim M \otimes N$.

Q.E.D.

Corollary 5.5. If $x \in \mathrm{r}_{\infty}(M, \Omega)$ then $M \sim M \otimes R_{x}$.

Lemma 5. 6. Given $0<x_{1}, x_{2} \leq 1$. Let $x_{1}=e^{-l_{1}}, x_{2}=e^{-l_{2}}$. If $l_{1} / l_{2}$ is rational then

$$
R_{x_{1}} \otimes R_{x_{2}} \sim R_{x}
$$

where $x=e^{-\left(l_{1}, l_{2}\right)}$ and $\left(l_{1}, l_{2}\right)$ is given by definition 3.12 .

Proof. By lemma 3.13 $x \in \mathrm{r}_{\infty}\left(R_{x_{1}} \otimes R_{x_{2}}\right)$, hence by corollary 5.5 $R_{x_{1}} \otimes R_{x_{2}} \sim\left(R_{x_{1}} \otimes R_{x_{2}}\right) \otimes R_{x}$. Since $\hat{\mathrm{r}}\left(R_{x_{1}} \otimes R_{x_{2}}\right) \subset S_{x}$, it follows from lemma 5.4 that $R_{x} \sim R_{x} \otimes\left(R_{x_{1}} \otimes R_{x_{2}}\right)$. Q.E.D.

The converse result to corollary 5.5 will be proved in lemma 5 . 8 . For this purpose we need the following rather lengthy lemma.

Lemma 5. 7. Given a finite type I factor $M$, a unit vector $\Omega$, $\frac{1}{2}<\lambda \leq 1, \varepsilon>0$, and operators $e_{12} \in M, f_{12} \in M^{\prime},\left\|e_{12}\right\|,\left\|f_{12}\right\| \leq 2$. Let $\left(\lambda_{1}, \lambda_{2}\right)=(\lambda, 1-\lambda), x=\lambda_{2} / \lambda_{1}, e_{21}=e_{12}^{*}, f_{21}=f_{12}^{*}$. Suppose that either of the following conditions hold:

(i) $\lambda=1, \varepsilon<\frac{1}{4}$ and

$$
\begin{aligned}
& \left\|e_{21} \Omega\right\|^{2}>1-\varepsilon \\
& \left\|e_{12} \Omega\right\|^{2}<\varepsilon
\end{aligned}
$$

(ii) $\quad \lambda \neq 1$ and

$$
\begin{gathered}
\left\|e_{21} \Omega\right\|^{2}>\lambda_{1}-\varepsilon \\
\left\|\lambda_{j}^{-1 / 2} e_{i j} \Omega-\lambda_{i}^{-1 / 2} f_{j i} \Omega\right\|^{2}<\varepsilon, \quad(i j)=(12) \text { or }(21)
\end{gathered}
$$

and $\varepsilon$ is sufficiently small that

$$
(1-\delta)^{3}>x^{1 / 2}
$$

and

$$
\begin{aligned}
\Delta & =\lambda-\varepsilon-9 \varepsilon \lambda / \delta^{2} \\
& =(\lambda-\varepsilon) / 2>\frac{1}{4}
\end{aligned}
$$


where

$$
\delta=(18 \lambda \varepsilon)^{1 / 2}(\lambda-\varepsilon)^{-1 / 2}<1 .
$$

Then there exist disjoint subsets $K^{1}, K^{2} \subset \operatorname{Sp}(\Omega / M)$ and a bijection $\phi$ from $K^{1}$ to $K^{2}$ such that

$$
\begin{gathered}
\max _{\mu \in K 1}\left|x^{1 / 2}-(\phi \mu / \mu)^{1 / 2}\right|<a \varepsilon^{1 / 2} \\
\mu\left(K^{1}\right)>b
\end{gathered}
$$

where $a, b$ are positive constants depending only on $\lambda$.

Proof. Let $H=H_{1} \otimes H_{2}$ where $M=\mathscr{B}\left(H_{1}\right) \otimes 1$. Then $e_{i j}=\hat{e}_{i j} \otimes 1$, $f_{i j}=1 \otimes \hat{f}_{i j} . \quad$ Let

$$
\Omega=\sum \rho_{\alpha}^{1 / 2} \Omega_{1 \alpha} \otimes \Omega_{2 \alpha}
$$

be a standard diagonal expansion of $\Omega$, and let $u_{\alpha \beta}, v_{\alpha \beta}$ be the associated standard matrix units for $M, M^{\prime}$ respectively.

We consider first the case $\lambda \neq 1$. Since $u_{\alpha \alpha} v_{\beta \beta}$ are a complete set of orthogonal projections, Eq. (5.14) gives

$$
\begin{gathered}
\varepsilon>\sum_{\alpha, \beta}\left\|u_{\alpha \alpha} v_{\beta \beta}\left(\lambda_{j}^{-1 / 2} e_{i j}-\lambda_{i}^{-1 / 2} f_{j i}\right) \Omega\right\|^{2} \\
=\sum\left|\left(\rho_{\beta} / \lambda_{j}\right)^{1 / 2}\left(\Omega_{1 \alpha}, \hat{e}_{i j} \Omega_{1 \beta}\right)-\left(\rho_{\alpha} / \lambda_{i}\right)^{1 / 2}\left(\Omega_{2 \beta}, \hat{f}_{j i} \Omega_{2 \alpha}\right)\right|^{2} .
\end{gathered}
$$

Interchanging $(\beta, j)$ and $(\alpha, i)$, taking the complex conjugate, and using $\left(\Omega_{1 \beta}, \hat{e}_{j i} \Omega_{1 \alpha}\right) *=\left(\Omega_{1 \alpha}, \hat{e}_{i j} \Omega_{1 \beta}\right)$ we get

$$
\sum\left|\left(\rho_{\alpha} / \lambda_{i}\right)^{1 / 2}\left(\Omega_{1 \alpha}, \hat{e}_{i j} \Omega_{1 \beta}\right)-\left(\rho_{\beta} / \lambda_{j}\right)^{1 / 2}\left(\Omega_{2 \beta}, \hat{f_{j i}} \Omega_{2 \alpha}\right)\right|^{2}<\varepsilon .
$$

Eqs. (5.20), (5.21) and the triangle inequality $\|x\|+\|y\| \geq\|x+y\|$ give

(5. 22) $\left\{\sum\left|\left(\rho_{\alpha} / \lambda_{i}\right)^{1 / 2}+\left(\rho_{\beta} / \lambda_{j}\right)^{1 / 2}\right|^{2}\left|\left(\Omega_{1 \alpha}, \hat{e}_{i j} \Omega_{1 \beta}\right)-\left(\Omega_{2 \beta}, \hat{f_{j i}} \Omega_{2 \alpha}\right)\right|^{2}\right\}^{1 / 2}<2 \varepsilon^{1 / 2}$ and thus

$$
\sum\left(\rho_{\beta} / \lambda_{j}\right)\left|\left(\Omega_{1 \alpha}, \hat{e}_{i j} \Omega_{1 \beta}\right)-\left(\Omega_{2 \beta}, \hat{f}_{i j} \Omega_{2 \alpha}\right)\right|^{2}<4 \varepsilon .
$$

Eq. (5.21) can be rewritten as

$$
\begin{aligned}
& \sum \mid\left\{\left(\rho_{\alpha} / \lambda_{i}\right)^{1 / 2}-\left(\rho_{\beta} / \lambda_{j}\right)^{1 / 2}\right\}\left(\Omega_{1 \alpha}, \hat{e}_{i j} \Omega_{1 \beta}\right) \\
& \quad+\left.\left(\rho_{\beta} / \lambda_{j}\right)^{1 / 2}\left\{\left(\Omega_{1 \alpha}, \hat{e}_{i j} \Omega_{1 \beta}\right)-\left(\Omega_{2 \beta}, \hat{f}_{i j} \Omega_{2 \alpha}\right)\right\}\right|^{2}<\varepsilon .
\end{aligned}
$$


Eqs. 5. (5.23), (5.24) and the triangle inequality give

(5. 25) $\sum \sum\left\{\left(\rho_{\alpha} / \lambda_{i}\right)^{1 / 2}-\left(\rho_{\beta} / \lambda_{j}\right)^{1 / 2}\right\}^{2}\left|\left(\Omega_{1 \alpha}, \hat{e}_{i j} \Omega_{1 \beta}\right)\right|^{2}<9 \varepsilon$.

Define

(5. 26) $L=\left\{(\alpha, \beta):\left|1-\left(\rho_{\alpha} \lambda_{1} / \rho_{\beta} \lambda_{2}\right)^{1 / 2}\right|<\delta, \rho_{\alpha} \neq 0, \rho_{\beta} \neq 0\right\}$.

Eq. (5.25) now gives

$$
\sum_{(\alpha, \beta) \notin L} \rho_{\beta}\left|\left(\Omega_{1 \alpha}, \hat{e}_{21} \Omega_{1 \beta}\right)\right|^{2}<9 \varepsilon \lambda_{1} / \delta^{2} .
$$

Since $\sum \rho_{\beta}\left|\left(\Omega_{1 \alpha}, \hat{e}_{21} \Omega_{1 \beta}\right)\right|^{2}=\left\|e_{21} \Omega\right\|^{2}$, Eqs. (5.27), (5.16) give

$$
\sum_{(\alpha, \beta) \in L} \rho_{\beta}\left|\left(\Omega_{1 \alpha}, \hat{e}_{21} \Omega_{1 \beta}\right)\right|^{2}>\Delta .
$$

Let

$$
L^{\prime}=\{\beta:(\alpha, \beta) \in L \text { for some } \alpha\} .
$$

For $\beta \in L^{\prime}$ we define

$$
\alpha(\beta)=\{\alpha:(\alpha, \beta) \in L\} .
$$

Let

$$
L^{\prime \prime}=\left\{\beta \in L^{\prime}: \sum_{\alpha \in \alpha(\beta)}\left|\left(\Omega_{1 \alpha}, \hat{e}_{21} \Omega_{1 \beta}\right)\right|^{2} \geq \Delta / 2\right\} .
$$

Using Eqs. (5.28), (5.30) and $\sum \rho_{\beta}=1$ we get

$$
\sum_{\beta \in L^{\prime \prime}} \sum_{\alpha \in \alpha(\beta)} \rho_{\beta}\left|\left(\Omega_{1 \alpha}, \hat{e}_{21} \Omega_{1 \beta}\right)\right|^{2}>1 / 2 \Delta .
$$

From $\left\|\hat{e}_{21}\right\| \leq 2, \quad \sum_{\alpha}\left|\left(\Omega_{1 \alpha}, \hat{e}_{21} \Omega_{1 \beta}\right)\right|^{2}=\left\|\hat{e}_{21} \Omega_{1 \beta}\right\|^{2} \leq 4$ and Eq. (5.31) we obtain

$$
\sum_{\beta \in L^{\prime \prime}} \rho_{\beta} \geq \frac{1}{4} \sum_{\beta \in L^{\prime \prime}} \sum_{\alpha} \rho_{\beta}\left|\left(\Omega_{1 \alpha}, \hat{e}_{21} \Omega_{1 \beta}\right)\right|^{2}>\frac{4}{8} .
$$

Let

(5. 33) $\quad K_{n}=\left\{\beta \in L^{\prime \prime}:(1-\delta)^{n}<\rho_{\beta}^{1 / 2} \leq(1-\delta)^{n-1}\right\}, n \in \mathrm{I}_{\infty}$.

Define $N$ by

$$
(1-\delta)^{N+1}<x^{1 / 2} \leq(1-\delta)^{N}
$$

Let

$$
\widehat{K}_{n}=\left\{\alpha \in \alpha(\beta): \beta \in K_{n}\right\} .
$$


It follows from Eqs. (5.26), (5.29) that $\alpha \in \alpha(\beta)$ implies

$$
\left|\rho_{\alpha}^{1 / 2}-x^{1 / 2} \rho_{\beta}^{1 / 2}\right|<x^{1 / 2} \rho_{\beta}^{1 / 2} \delta .
$$

Since $0<\delta<1$ we have $(1+\delta)<(1-\delta)^{-1}$ and thus

$$
(1-\delta) x^{1 / 2} \rho_{\beta}^{1 / 2}<\rho_{\alpha}^{1 / 2}<(1+\delta) x^{1 / 2} \rho_{\beta}^{1 / 2}<(1-\delta)^{-1} x^{1 / 2} \rho_{\beta}^{1 / 2} .
$$

It now follows from Eqs. (5.33), (5.34) that

$$
\widehat{K}_{n} \subset \cup_{m=-2}^{+2} K_{n+N+m} .
$$

Consider an arbitrary $K \subset L^{\prime \prime}$. Let $\widehat{K}=\{\alpha \in \alpha(\beta): \beta \in K\}$. Let $N(K)$, $N(\widehat{K})$ be the number of elements in $K, \widehat{K}$ respectively. Using Eq. (5.30) and $\left\|\hat{e}_{21}\right\| \leq 2$ we get

$$
\begin{aligned}
\frac{1}{2} \Delta N(K) & \leq \sum_{\beta \in K} \sum_{\alpha \in \alpha(\beta)}\left|\left(\Omega_{1 \alpha}, \hat{e}_{21} \Omega_{1 \beta}\right)\right|^{2} \\
& \leq \sum_{\beta \in K} \sum_{\alpha \in \widehat{K}}\left|\left(\Omega_{1 \alpha}, \hat{e}_{21} \Omega_{1 \beta}\right)\right|^{2} \\
& \leq \sum_{\alpha \in \widehat{K}}\left\|\hat{e}_{21}^{*} \Omega_{1 \alpha}\right\|^{2} \leq 4 N(\widehat{K}) .
\end{aligned}
$$

We now construct subsets $K_{n}^{1} \subset K_{n}, K_{n}^{2} \subset \widehat{K}_{n}$ and a bijection $\phi_{n}$ from $K_{n}^{1}$ to $K_{n}^{2}$ such that $\phi_{n} \beta \in \alpha(\beta)$. If $K_{n}$ is nonempty, we order the elements in $K_{n}, \widehat{K}_{n}$ respectively by

$$
\rho_{\beta_{n 1}} \geq \rho_{\beta_{n 2}} \geq \cdots \geq \rho_{\beta_{n, N}\left(K_{n}\right)}
$$

and

$$
\rho_{\alpha_{n 1}} \geq \rho_{\alpha_{n 2}} \geq \cdots \geq \rho_{\alpha_{n, N(\widehat{K} n)}} .
$$

Since $\alpha_{n 1} \in \widehat{K}_{n}$ we have $\alpha_{n 1} \in \alpha\left(\beta_{n k}\right)$ for some $\beta_{n k} \in K_{n}$. Since Eq. (5.24) implies that $\alpha\left(\beta_{n 1}\right)$ is nonempty, there exists some $\alpha_{n j} \in \alpha\left(\beta_{n 1}\right)$. Thus

$$
\rho_{\alpha_{n 1}} / \rho_{\beta_{n k}} \geq \rho_{\alpha_{n 1}} / \rho_{\beta_{n 1}} \geq \rho_{\alpha_{n j}} / \rho_{\beta_{n 1}}
$$

and it follows from Eq. (5.26) that $\alpha_{n 1} \in \alpha\left(\beta_{n 1}\right)$. We include $\beta_{n 1} \in K_{n}^{1}$ and define $\phi_{n} \beta_{n 1}=\alpha_{n 1}$. Let $m$ be the smallest integer such that $m \geq 8 / \Delta$. Let $p_{n}$ be the largest integer such that $p_{n} m<N\left(K_{n}\right)$. If $p_{n} \leqslant 0$ we are done. If $p_{n}>0$ we proceed as follows. Assume that $\alpha_{n j} \notin \alpha\left(\beta_{n, m+1}\right)$ for any $j>1$. Since $\alpha\left(\beta_{n, m+1}\right)$ is nonempty by Eq. 
(5. 30), we must have $\alpha\left(\beta_{n, m+1}\right)=\left\{\alpha_{n 1}\right\}$. It follows from Eq. : (5.37) applied to $K=\left\{\beta_{n 1}, \beta_{n 2}, \cdots \beta_{n, m+1}\right\}$ that $\widehat{K}$ must contain at least two elements. Thus there exists some $\alpha_{n j} \in \alpha\left(\beta_{n, k}\right)$ for some $j>1$ and $k \leq m$. We have

$$
\rho_{\alpha_{n 1}} / \rho_{\beta_{n, m+1}} \geq \rho_{\alpha_{n j}} / \rho_{\beta_{n, m+1}} \geq \rho_{\alpha_{n,}} / \rho_{\beta_{n k}}
$$

and it follows from Eq. (5.26) that $\alpha_{n j} \in \alpha\left(\beta_{n, m+1}\right)$ which is a contradiction. Thus $\alpha\left(\beta_{n, m+1}\right)$ must contain some $\alpha_{n j}, j>1$. Let $j(n, 1)$ be the smallest $j>1$ such that $\alpha_{n j} \in \alpha\left(\beta_{n, m+1}\right)$. By a repetition of this argument we obtain a sequence

$$
1<j(n, 1)<j(n, 2)<\cdots<j\left(n, p_{n}\right)
$$

such that

$$
\alpha_{n, j(n, k)} \in \alpha\left(\beta_{n, k m+1}\right), k=1, \cdots p_{n} .
$$

We include $\beta_{n, m+1}, \beta_{n, 2 m+1}, \cdots \beta_{n, p_{n} m+1} \in K_{n}^{1}$ and define

$$
\phi_{n} \beta_{n, k m+1}=\alpha_{n, j(n, k)}, k=1, \cdots p_{n} .
$$

It follows from Eq. (5.26) that

$$
\max _{\beta \in K_{n}^{1}}\left|x^{1 / 2}-\left(\rho_{\beta} / \rho_{\phi_{n} \beta}\right)^{1 / 2}\right|<\delta x^{1 / 2} .
$$

By construction $K_{n}^{1}$ contains at least $N\left(K_{n}\right) / m$ elements and

$$
\sum_{\beta \in K_{n}^{1}} \rho_{\beta} \geq m^{-1} \sum_{\beta \in K_{n}} \rho_{\beta} .
$$

We now proceed to define the desired sets $K^{1}, K^{2}$. Since the $K_{n}$ defined by Eq. (5.33) are mutually disjoint, it follows that the $K_{n}^{1}$ are mutually disjoint. We note that Eq. (5.15) implies that $N \geq 3$ where $N$ is defined by Eq. (5.34). It then follows from Eq. (5.36) that $\widehat{K}_{n}$ and $K_{p}$ are disjoint if $p \leq n$. By induction on $n$, we define $K^{1}$ to contain all $\rho_{\beta}, \beta \in K_{n}^{1}, n \in I_{\infty}$ such that for any $\beta^{\prime} \in K_{p}^{1}, p<n$ and $\beta^{\prime} \in K^{1}$ the conditions

$$
\begin{aligned}
& \beta \neq \phi_{p} \beta^{\prime} \\
& \phi_{n} \beta \neq \phi_{p} \beta^{\prime}
\end{aligned}
$$

are satisfied. We define $\phi \rho_{\beta}=\rho_{\phi_{n} \beta}$. Eq. (5.47) eliminates at most one 
$\beta$ for each $\beta^{\prime}$ taken into $K^{1}$ and thus at most half of the $\beta$ which would be otherwise available. It follows from Eq. (5.36) that Eq. (5.48) eliminates at most 4 of the available $\rho_{\beta}$ for each $\rho_{\beta^{\prime}}$ taken into $K^{1}$ and hence at most $4 / 5$ of the $\rho_{\beta}$ which would be otherwise available. The net effect of these two conditions is to reduce the total number of available $\rho_{\beta}$ by a factor of at most 10 . Since the eliminated $\rho_{\beta}$ are never larger than the $\rho_{\beta^{\prime}}$ taken into $K^{1}$, it follows from Eq. (5.32) that

$$
\sum_{\rho_{\beta} \subseteq K 1} \rho_{\beta}>(\Delta / 8) m^{-1} / 10 \text {. }
$$

Since $\Delta>1 / 4$ by Eq. (5.16) we have $m \leq 32$ and thus

$$
\sum_{\rho_{\beta} \in K^{1}} \rho_{\beta}>1 / 10240 \text {. }
$$

Thus Eqs. (5.18), (5.19) are satisfied by Eqs. (5.45), (5.49) and the proof is complete for the case $\lambda \neq 1$.

We now consider the case $\lambda=1$. Let

$$
L=\left\{(\alpha, \beta): \rho_{\alpha} \leq 2 \varepsilon \rho_{\beta}, \rho_{\beta} \neq 0, \Omega_{1 \alpha} \neq 0\right\} .
$$

Using Eq. (5.12) we have

$$
\varepsilon>\left\|e_{12} \Omega\right\|^{2}=\sum \rho_{\alpha}\left|\left(\Omega_{1 \alpha}, \hat{e}_{21} \Omega_{1 \beta}\right)\right|^{2}
$$

and thus

$$
\varepsilon>\sum_{(\alpha, \beta) \mp L} \rho_{\alpha}\left|\left(\Omega_{1 \alpha}, \hat{e}_{21} \Omega_{1 \beta}\right)\right|^{2}>2 \varepsilon \sum_{(\alpha, \beta) \Subset L} \rho_{\beta}\left|\left(\Omega_{1 \alpha}, \hat{e}_{21} \Omega_{1 \beta}\right)\right|^{2} .
$$

Using Eq. (5.11) we have

$$
1-\varepsilon<\left\|e_{21} \Omega\right\|^{2}=\sum \rho_{\beta} \mid\left(\Omega_{1 \alpha}, \hat{e}_{21} \Omega_{1 \beta}\right) !^{2}
$$

and it follows that

$$
\sum_{(\alpha, \beta) \in L} \rho_{\beta}\left|\left(\Omega_{1 \alpha}, \hat{e}_{21} \Omega_{1 \beta}\right)\right|^{2}>\gamma=1 / 2-\varepsilon>1 / 4 .
$$

Let

$$
\begin{aligned}
& L^{\prime}=\{\beta:(\alpha, \beta) \in L \text { for some } \alpha\} \\
& L^{\prime \prime}=\left\{\beta \varepsilon L^{\prime}: \sum_{\alpha \in \alpha(\beta)}\left|\left(\Omega_{1 \alpha}, \hat{e}_{21} \Omega_{1 \beta}\right)\right|^{2} \geq \gamma / 2\right\} .
\end{aligned}
$$

Using Eqs. (5.51), (5.52) and $\sum \rho_{\beta}=1$ we get

$$
\sum_{\beta \in L^{\prime \prime}} \sum_{\alpha \in \alpha(\beta)} \rho_{\beta}\left|\left(\Omega_{1 \alpha}, \hat{e}_{21} \Omega_{1 \beta}\right)\right|^{2}>\gamma / 2
$$

and thus 


$$
\sum_{\beta \in L^{\prime \prime}} \rho_{\beta}>\gamma / 8
$$

since $\left\|\hat{e}_{21}\right\| \leq 2$. Given any $K \subset L^{\prime \prime}$, let $\widehat{K}=\{\alpha \in \alpha(\beta): \beta \in K\}$ and let $N(K)$ be the number of elements in $K$. By the same argument used to derive Eq. (5.37) it follows that $\widehat{K}$ contains at least $\gamma N(K) / 8$ elements. Let $m$ be the smallest integer such that $m \geq 8 \gamma^{-1}$. We order the elements in $L^{\prime \prime}$ by $\rho_{\beta_{1}} \geq \rho_{\beta_{2}} \geq \cdots$. We define $K^{0}$ to contain $\rho_{\beta_{1}}, \rho_{\beta_{m+1}}, \rho_{\beta_{2 m+1}}, \cdots$. By the same argument which follows Eq. (5.39) we can obtain elements $\alpha_{n} \in \alpha\left(\beta_{n m+1}\right)$ such that $\alpha_{n} \neq \alpha_{j}$ for any $j<n$. We define $\phi \rho_{\beta n m-1}=\rho_{\alpha_{n}}$. By induction on $n$, we define $K^{1}$ to contain all $\rho_{\beta_{n n+1}}$ such that $\rho_{\beta_{n n+1}} \neq \phi \rho_{\beta^{\prime} m+1}$ in $\operatorname{Sp}(\Omega / K)$ for any $j<n$. This condition eliminates at most one $\rho_{\beta}$ for each $\rho_{\beta^{\prime}}$ taken into $K^{1}$, and thus at most half of the $\rho_{\beta}$ which would be otherwise available. Since the eliminated $\rho_{\beta}$ are never larger than the $\rho_{\beta^{\prime}}$ taken into $K^{1}$, it follows from Eq. (5.53) that

$$
\sum_{\rho \in K^{1}} \rho \geq m^{-1} \gamma / 16>1 / 2048
$$

(where we used Eq. (5.51) to obtain $m \leq 32$ ). Thus Eq. (5.19) is satisfied. We define $K^{2}=\left\{\phi \rho: \rho \in K^{1}\right\}$. It follows from Eq. (5.50) that

$$
\max _{\rho \in K^{1}} \phi \rho / \rho \leq 2 \varepsilon
$$

and thus Eq. (5.18) is satisfied.

Q.E.D.

Lemma 5.8. Given $M=\mathrm{R}\left(M_{\nu}, \Omega_{\nu}\right)$. Then $M \sim M \otimes R_{x}, 0 \leq x \leq 1$ implies that $x \in \mathrm{r}_{\infty}(M, \Omega)$.

Proof. By lemma 3.15 we can assume that $M$ has a cyclic and separating vector. Since $R_{x}$ has a cyclic and separating vector, we can assume that $M \otimes R_{x}$ has a cyclic and separating vector. Then $M \sim$ $M \otimes R_{x}$ implies that $M \stackrel{u}{\sim} M \otimes R_{x}$ and we have

$$
\begin{aligned}
& H=H_{M} \otimes H_{R} \\
& H_{R}=\bigotimes_{n=1}^{\infty}\left(H_{n 1} \otimes H_{n 2}, \Phi_{n}\right) \\
& R=\left(\bigcup_{n=1}^{\infty} R_{n 1}\right)^{\prime \prime}
\end{aligned}
$$




$$
R_{n i}=\mathbf{1}_{M} \otimes\left[\left(\bigotimes_{m \neq n} \mathbb{1}_{m}\right) \otimes\left(\mathscr{B}\left(H_{n i}\right) \otimes \mathbf{1}\right)\right], \quad i=1,2
$$

where $\operatorname{dim} H_{n 1}=\operatorname{dim} H_{n 2}=2, \operatorname{Sp}\left(\Phi_{n} / \mathscr{B}\left(H_{n 1}\right) \otimes \mathbb{1}\right)=(\lambda, 1-\lambda)$ and $x=$ $(1-\lambda) / \lambda$. Let

$$
\Phi_{n}=\lambda^{1 / 2} \Phi_{11}^{n} \otimes \Phi_{21}^{n}+(1-\lambda)^{1 / 2} \Phi_{12}^{n} \otimes \Phi_{22}^{n}
$$

be a standard diagonal expansion of $\Phi_{n}$, and let $u_{i j}^{n}, v_{i j}^{n}$ be the associated standard matrix units for $R_{n 1}, R_{n 2}$ respectively. Choose some unit vector $\Phi_{M} \in H_{M}$ and let $\Phi=\Phi_{M} \otimes\left(\bigotimes_{n=1}^{\infty} \Phi_{n}\right)$. It follows from lemma 2.7 that for any $Q_{n} \in\left\{R_{n 1}, R_{n 2}\right\}^{\prime \prime},\left\|Q_{n}\right\|<N$ for some fixed $N<\infty$, that

$$
\lim _{n \rightarrow \infty}\left\{\left\|Q_{n} \Omega\right\|-\left\|Q_{n} \Phi\right\|\right\}=0
$$

Case (i), $x \neq 0,1$. Define

$$
Q_{i j}^{n}=\lambda_{j}^{-1 / 2} u_{i j}^{n}-\lambda_{i}^{-1 / 2} v_{j i}^{n},(i j)=(12) \text { or }(21)
$$

where $\left(\lambda_{1}, \lambda_{2}\right)=(\lambda, 1-\lambda)$. Then

$$
Q_{i j}^{n} \Phi=0 \text {. }
$$

Also

$$
\left\|u_{21}^{n} \Phi\right\|^{2}=\lambda
$$

Let $\varepsilon_{m}>0, \varepsilon_{m} \rightarrow 0$. It follows from Eqs. (5.61-64) that we can choose a subsequence $n(m), m \in I_{\infty}$, such that

$$
\begin{aligned}
& \left\|Q_{i j}^{n(m)} \Omega\right\|<\varepsilon_{m} \\
& \left|\lambda-\left\|u_{21}^{n(m)} \Omega\right\|^{2}\right|<\varepsilon_{m} .
\end{aligned}
$$

We have $M=\bigotimes_{\nu \in A} M_{\nu}, M^{\prime}=\bigotimes_{\nu \in A} M_{\nu}^{\prime}$. We will prove that there exist mutually disjoint finite subsets $J_{m} \subset A$, and $e_{i j}^{m} \in M\left(J_{m}\right), f_{i j}^{m} \in M^{\prime}\left(J_{m}\right)$, $\left\|e_{i j}^{m}\right\|,\left\|f_{i j}^{m}\right\| \leq 2$ satisfying

$$
\begin{aligned}
& \left\|\left(e_{i j}^{m}-u_{i j}^{n(m)}\right) \Omega\right\|<\varepsilon_{m} \\
& \left\|\left(f_{i j}^{m}-v_{i j}^{n(m)}\right) \Omega\right\|<\varepsilon_{m}
\end{aligned}
$$

where $(i j)=(12)$, (21) and $\left(e_{i j}^{m}\right)^{*}=e_{j i}^{m},\left(f_{i j}^{m}\right)^{*}=f_{j i}^{m}$. For $m=1$, this follows from Kaplansky's density theorem ${ }^{1)}$ applied to the hermitean and antihermitean parts of $u_{i j}^{n(m)}, v_{i j}^{n(m)}$. Assume that $J_{m}, e_{i j}^{m}, f_{i j}^{m}$ exist for

1) Dixmier [8], Sec. I.3. 
$m=1, \cdots k-1$. Let $J^{k}=\bigcup_{m=1}^{k-1} J_{m}$. Then we have $M=M\left(A-J^{k}\right) \otimes M\left(J^{k}\right)$ where $M\left(J^{k}\right)$ is a finite type I factor. If $M$ is infinite then $M \sim M$ $\left(A-J^{k}\right)$ by lemma 5.2 . If $M$ is finite then both $M$ and $M\left(A-J^{k}\right)$ must be hyperfinite $I I_{1}$ and again we have $M \sim M\left(A-J^{k}\right)$. By assumption $M$ and $M\left(A-J^{k}\right)$ have a cyclic and separating vector, hence $M \stackrel{u}{\sim} M\left(A-J^{k}\right)$. Thus we can repeat the above argument with $M$ replaced by $M\left(A-J^{k}\right)$ to obtain $J_{k}, e_{i j}^{k}, f_{i j}^{k}$. It follows from Eqs. (5.65-68) that

$$
\begin{gathered}
\left\|\left(\lambda_{j}^{-1 / 2} e_{i j}^{m}-\lambda_{i}^{-1 / 2} f_{j i}^{m}\right) \Omega\right\|<\varepsilon_{m}\left[1+\lambda^{-1 / 2}+(1-\lambda)^{-1 / 2}\right] \\
\left|\lambda-\left\|e_{21}^{m} \Omega\right\|^{2}\right|<2 \varepsilon_{m} .
\end{gathered}
$$

Since $\varepsilon_{m} \rightarrow 0$, it follows from lemma 5.7 that for $m>N$ there exist $K_{m}^{1}, K_{m}^{2} \subset \operatorname{Sp}\left(\Omega\left(J_{m}\right) / M\left(J_{m}\right)\right)$ and a bijection $\phi_{m}$ from $K_{m}^{1}$ to $K_{m}^{2}$ such that $\left(I_{m}, K_{m}^{i}, \phi_{m}, m>N\right)$ is an $x$-sequence.

Case (ii), $x=0$. Retain Eq. (5.64) and replace Eq. (5.63) by

$$
u_{12}^{n} \Phi=0 \text {. }
$$

Then choose a subsequence $n(m)$ such that Eq. (5.66) holds and

$$
\left\|u_{12}^{n(m)} \Omega\right\|<\varepsilon_{m} .
$$

The remainder of the argument is a straightforward repetition of the above argument, and we omit the details.

Case (iii), $x=1$. Since $R_{1}$ is type $I I_{1}, M \sim M \otimes R_{1}$ implies that $M$ is not type I. Lemmas 2.14 and 3.8 then imply that $1 \in \mathrm{r}_{\infty}(M)$.

Q. E. D.

Theorem 5.9. The asymptotic ratio set $\mathrm{r}_{\infty}(M)$ given by definition 3.2 for ITPFI factors $M$, is an algebraic invariant of $M$.

Proof. By corollary 5.5 and lemma 5.8, $x \in \mathrm{r}_{\infty}(M), 0 \leq x \leq 1$ if and only if $M \sim M \otimes R_{x}$. By lemma 3.6, $x \in \mathrm{r}_{\infty}(M), x>1$ if and only if $x^{-1} \in \mathrm{r}_{\infty}(M)$.

Q. E. D.

We recall that $M=\mathrm{R}\left(M_{\nu}, \Omega_{\nu}\right)$ where $M_{\nu}$ is type $I_{n_{\nu}}, 2 \leq n_{\nu} \leq \infty$ is an ITPFI factor (corollary 2.9). We now show that all non-zero $x \in$ $\mathrm{r}_{\infty}(M)$ can be calculated directly from definition 3.2 even if some of the $n_{\nu}$ are infinite. It should be noted that a direct application of de- 
finition 3.2 may fail to give a 0 -sequence, even though $0 \in \mathrm{r}_{\infty}(M)$. Consider $M=I_{\infty} \otimes R_{1}$, then $0 \in \mathrm{r}_{\infty}(M)$ but the only $x$-sequences allowed are $x=1$. However this is not a problem since if some $n_{\nu}=\infty$, then we necessarily have $0 \in \mathrm{r}_{\infty}(M)$ since $M$ is infinite.

Lemma 5.10. Given $M=\mathrm{R}\left(M_{\nu}, \Omega_{\nu}\right)$ where $M_{\nu}$ is type $I_{n_{\nu}}, 2 \leq n_{\nu}$ $\leq \infty$ and $n_{\nu}=\infty$ for some $\nu$. Then $\mathrm{r}_{\infty}(M)$ contains 0 and all $x \in(0, \infty)$ for which there exists an $x$-sequence satisfying the conditions of definition 3.2.

Proof. By assumption $n_{\nu}=\infty$ for some $\nu$ and $M$ is infinite. By lemma 5.2 $M \sim M \otimes R_{0}$ and $0 \in \mathrm{r}_{\infty}(M)$. It remains to show that $x \in \mathrm{r}_{\infty}(M), x \neq 0$ if and only if there exists an $x$-sequence for $M$.

Let $\operatorname{Sp}\left(\Omega_{\nu} / M_{\nu}\right)=\left\{\lambda_{\nu j}\right\}$. By lemma 2.14, $M$ is type $I_{\infty}$ if and only if $\sum\left(1-\lambda_{\nu 1}\right)<\infty$. By lemma 3.8, $\mathrm{r}_{\infty}(M)=S_{0}$ if and only if $M$ is type $I_{\infty}$ if $M$ is an ITPFI factor. Thus $\sum\left(1-\lambda_{v 1}\right)=\infty$ if and only if $1 \in \mathrm{r}_{\infty}(M)$. Since the proof of lemma 3.8 remains valid even if $n_{\nu}=\infty$ is allowed, $\sum\left(1-\lambda_{\nu 1}\right)=\infty$ if and only if there is a 1 -sequence for $M$. Thus it remains only to consider $x \neq 0,1$.

We construct a projection $P \in M$ such that $M_{P}=\mathrm{R}\left(M_{\nu}, \Omega_{\nu}^{\prime}\right)$ has $x$ sequences if and only if $\mathrm{R}\left(M_{\nu}, \Omega_{\nu}\right)$ has $x$-sequences, $x \neq 0,1$. For each $\nu$ choose $m_{\nu}<\infty, m_{\nu} \leq n_{\nu}$ such that

$$
\sum_{j=m_{\nu}+1}^{n_{\nu}} \lambda_{\nu j}<2^{-(\nu+2)}\left\|\Omega_{\nu}\right\|
$$

Let

$$
\Omega_{\nu}=\sum \lambda_{\nu j}^{1 / 2} \psi_{\nu 1 j} \otimes \psi_{\nu 2 j}
$$

be a standard diagonal expansion of $\Omega_{\nu}$. Define $P_{\nu} \otimes 1 \in M_{\nu}$ by

$$
P_{\nu} \psi_{\nu 1 j}= \begin{cases}\psi_{\nu 1 j} & \text { if } j \leq m_{\nu} \\ 0 & \text { if } j>m_{\nu}\end{cases}
$$

and let $P=\otimes_{\nu}\left(P_{\nu} \otimes 1\right) \in M$. We have

$$
\|P \Omega\|^{2}=\Pi\left[\left\|\Omega_{\nu}\right\|^{2}-\sum_{j=m_{\nu}+1}^{n_{\nu}} \lambda_{\nu j} \mid>0\right.
$$

where we used Eq. (5.73). We have $M_{P}=\mathrm{R}\left(M_{\nu}, \Omega_{\nu}^{\prime}\right)$ where $\operatorname{Sp}\left(\Omega_{\nu}^{\prime} / M_{\nu}\right)=$ $\left(\lambda_{\nu 1}, \cdots \lambda_{\nu m_{\nu}}\right)$. Let $\left(I_{n} K_{n}^{i}, \phi_{n}\right)$ be an $x$-sequence for $\mathrm{R}\left(M_{\nu}, \Omega_{\nu}^{\prime}\right)$. Clearly 
$\left(I_{n}, K_{n}^{i}, \phi_{n}\right)$ is also an $x$-sequence for $\mathrm{R}\left(M_{\nu}, \Omega_{\nu}\right)$. Conversely let $\left(I_{n}, K_{n}^{i}, \phi_{n}\right)$ be an $x$-sequence for $\mathrm{R}\left(M_{\nu}, \Omega_{\nu}\right), x \neq 0$. It follows from definition 3.2 and Eq. (5.73) that we can omit all $\mu \in K_{n}^{i}, i=1,2$, $n \in I_{\infty}$ which contain some $\lambda_{v j}, j>m_{\nu}$ as a factor. The argument is then reversible, and there exists an $x$-sequence for $\mathrm{R}\left(M_{\nu}, \Omega_{\nu}^{\prime}\right)$. By lemma 5.2, $M \sim M_{P} \otimes R_{0}$. Hence $\mathrm{r}_{\infty}\left(M_{P}\right) \subset \mathrm{r}_{\infty}(M)$ and $x \notin \mathrm{r}_{\infty}(M)$ implies that $\mathrm{R}\left(M_{\nu}, \Omega_{\nu}^{\prime}\right)$ does not have an $x$-sequence. Conversely, if $x \in \mathrm{r}_{\infty}(M), x \neq 0,1$, then $M$ cannot be $R_{1} \otimes R_{0}$. Hence $M_{P}$ cannot be a finite factor and by lemma $5.2 M \sim M_{P} \otimes R_{0} \sim M_{P}$, which implies that $M_{P}=\mathrm{R}\left(M_{\nu}, \Omega_{\nu}^{\prime}\right)$ has an $x$-sequence.

\section{Asymptotic Ratio Set for Arbitrary Factors}

In the preceding section we proved that for ITPFI factors $M$, $x \in \mathrm{r}_{\infty}(M), 0 \leq x \leq 1$ if and only if $M \sim M \otimes R_{x}$. In this section we use this result to extend the definition of $\mathrm{r}_{\infty}(M)$ to arbitrary $M$. We give some properties of $\mathrm{r}_{\infty}(M)$ for the general case.

Definition 6.1. Let $M$ be any factor. We define $\mathrm{r}_{\infty}(M)$ by $x \in \mathrm{r}_{\infty}(M), 0 \leq x \leq 1$ if and only if $M \sim M \otimes R_{x}$. If $x \in \mathrm{r}_{\infty}(M), x \neq 0$ we include $x^{-1} \in \mathrm{r}_{\infty}(M)$.

We shall need the following result which is due to Sakai [16].

Lemma 6.2. Let $M, N$ be factors. If $M$ is type III then $M \otimes N$ is a type III factor.

Lemma 6.3. $M$ is finite if and only if $\mathrm{r}_{\infty}(M)=\phi$ or $S_{1} . \quad M$ is type III if $x \in \mathrm{r}_{\infty}(M)$ for some $0<x<1$.

Proof. If $\mathrm{r}_{\infty}(M)=\phi$ or $S_{1}$ then $0 \notin \mathrm{r}_{\infty}(M)$ and $M$ is finite by lemma 5.2. Since $R_{x}$ is infinite if $x \neq 1, M$ finite implies that $M \propto M \otimes R_{x}$ if $x \neq 1$ and hence $\mathrm{r}_{\infty}(M)=\phi$ or $S_{1}$. If $x \in \mathrm{r}_{\infty}(M)$ for some $0<x<1$ then we have $M \nsim M \otimes R_{x}$ where $R_{x}$ is type III. It follows from lemma 6.2 that $M$ is type III.

Q.E. D.

Schwartz [17] has given a type $\mathrm{II}_{1}$ factor $M$ such that $M$ is nonisomorphic to $M \otimes R_{1}$. Thus there exists a type $\mathrm{II}_{1}$ factor $M$ with 
$\mathrm{r}_{\infty}(M)=\phi$

Lemma 6.4. $\mathrm{r}_{\infty}(M \otimes N) \supset \mathrm{r}_{\infty}(M) \cup \mathrm{r}_{\infty}(N)$

Proof. $\quad M \sim M \otimes R_{x}$ implies $M \otimes N \sim M \otimes N \otimes R_{x}$. $\quad$ Q. E. D.

Lemma 6.5. If $\mathrm{r}_{\infty}(M)-\{0\}$ is nonempty, then it is a multiplicative subgroup of $(0, \infty)$.

Proof. By definition $x^{-1} \in \mathrm{r}_{\infty}(M)-\{0\}$ if and only if $x \in \mathrm{r}_{\infty}(M)$ $-\{0\}$. For $x \in(0, \infty)$ define

$$
\alpha(x)= \begin{cases}x & 0<x \leq 1 \\ x^{-1} & 1<x<\infty\end{cases}
$$

Let $x, y \in \mathrm{r}_{\infty}(M)-\{0\}$. Then we have $M \sim M \otimes R_{\alpha(x)}, \quad M \sim M \otimes R_{\alpha(y)}$ which implies that $M \sim M \otimes\left(R_{\alpha(x)} \otimes R_{\alpha(y)}\right)$. Since $x, y \in \mathrm{r}_{\infty}\left(R_{\alpha(x)} \otimes R_{\alpha(y)}\right)$ it follows from lemma 3.6 that $x y \in \mathrm{r}_{\infty}\left(R_{\alpha(x)} \otimes R_{\alpha(y)}\right)$. Thus $M \sim M \otimes$ $R_{\alpha(x y)}$ and $x y \in \mathrm{r}_{\infty}(M)-\{0\}$ Q. E. D.

Lemma 6.6. Given $x_{1}, x_{2} \in \mathrm{r}_{\infty}(M), 0<x_{1}, x_{2}<1$. Let $x_{i}=e^{-l_{4}}, i$ $=1,2$. If $l_{1} / l_{2}$ is irrational then $\mathrm{r}_{\infty}(M)=S_{\infty}$. If $l_{1} / l_{2}$ is rational then $e^{-\left(l_{1}, l_{2}\right)} \in \mathrm{r}_{\infty}(M)$ (see definition 3.12).

Proof. If $x_{1}, x_{2} \in \mathrm{r}_{\infty}(M)$ then $M \sim M \otimes R_{x_{1}} \sim M \otimes R_{x_{1}} \otimes R_{x_{2}} . \quad$ By lemmas 3.13 and 5.6 we have $\mathrm{r}_{\infty}\left(R_{x_{1}} \otimes R_{x_{2}}\right)=S_{x}$ where $x=\infty$ if $l_{1} / l_{2}$ is irrational and $x=e^{-\left(l_{1}, l_{2}\right)}$ otherwise. We have $R_{x_{1}} \otimes R_{x_{2}} \sim R_{x_{1}} \otimes R_{x_{2}} \otimes R_{y}$ for any $y \in S_{x}$. It follows that $M \sim M \otimes R_{x_{1}} \otimes R_{x_{2}} \otimes R_{y} \sim M \otimes R_{y}$ and hence $y \in \mathrm{r}_{\infty}(M)$.

Q. E. D.

Definition 6.7. Given $0<x<1$. Let $K$ denote some sequence $\left\{k_{\nu} ; \nu \in I_{\infty}\right\}$ where $k_{\nu}$ is either a positive integer or $\infty$. Let $p_{1}<p_{2}<\cdots$ be the set of all prime numbers. We define $S(x, K)$ as the subset of $[0, \infty)$ containing 0 and $x^{n / m}$ for all integers $n$ and all integers $m=$ $\Pi_{\nu} p_{\nu}^{n_{\nu}}$ where $0 \leq n_{\nu}<k_{\nu}$.

Corollary 6.8. For any factor $M, \mathrm{r}_{\infty}(M)$ is one of the following sets: the empty set $\phi, S_{0}, S_{01}, S_{1}, S_{\infty}$ or $S(x, K)$ for some $0<x<1$ and some $K$.

Proof. Follows immediately from lemmas 6.5 and 6.6. Q. E. D. 
We note that if $K=\left\{k_{\nu}\right\}$ where $k_{\nu}=1$ for all $\nu$, then $S(x, K)$ $=S_{x}$. If it can be proved that $\mathrm{r}_{\infty}(M)$ is closed, then $\mathrm{r}_{\infty}(M)$ must be one of the sets $\phi, S_{x}(0 \leq x \leq 1), S_{\infty}, S_{01}$. The following lemma was obtained as a result of our effort, as yet unsuccessful, to prove that $\mathrm{r}_{\infty}(M)$ is closed.2 ${ }^{2}$

Lemma 6.9. Let $M$ be a factor and $x_{n} \in \mathrm{r}_{\infty}(M), n=1,2, \cdots \cdots, 0<$ $x_{n}<1, \lim x_{n}=x$. Then there exist subfactors $M_{n}$ of $M$ and $N_{n}$ of $M^{\prime}$ and a vector $\chi$ such that

(i) $\quad M_{n} \sim R_{x_{n}} \sim M_{n}$.

(ii) $\quad M_{a}=\left(\cup_{n} M_{n}\right)^{\prime \prime}$ and $N_{a}=\left(\cup_{n} N_{n}\right)^{\prime \prime}$ are factors isomorphic to $\underset{n}{\otimes} R_{x_{n}}$.

(iii) $H_{a} \equiv \overline{M_{a} \chi}=\overline{N_{a} \chi}$.

(iv) $\chi$ is separating for $M$ and $M^{\prime}$.

(v) Restricted to $H_{a},\left\{H_{a}, \chi, M_{n}, N_{n}, n=1,2, \cdots\right\}$ are unitarily equivalent to $\left\{\left(\otimes H_{m}\right),\left(\otimes x_{m}\right), R_{x_{n}}, R_{x_{n}}^{\prime}, n=1,2, \cdots\right\}$ where $R_{x_{n}}^{\prime}$ is the commutant of $R_{x_{n}}$ in $H_{n}$ and $\chi_{n}$ is a cyclic and separating vector of $R_{x_{n}}$ in $H_{n}$.

(vi) Let $M_{b}=M_{a}^{\prime} \cap M, N_{b}=N_{a}^{\prime} \cap M^{\prime} . \quad M_{0} \equiv\left(M_{a} \cup M_{b}\right)^{\prime \prime}$ and $N_{0} \equiv$ $\left(N_{a} \cup N_{b}\right)^{\prime \prime}$ have the property that $M=M_{0} \oplus M_{c}$ and $M^{\prime}=$ $N_{0} \oplus N_{c}$ where $M_{c}$ and $N_{c}$ brings $\overline{M_{0} N_{0} \chi}$ to its orthogonal complement.

(vii) Restricted to $\overline{M_{0} N_{0} \chi}, M_{0}=R_{a} \otimes R_{b}, N_{0}=R_{a}^{\prime} \otimes R_{b}^{\prime}, M_{a}=R_{a} \otimes 1, M_{b}$ $=1 \otimes R_{b}, N_{a}=R_{a}^{\prime} \otimes 1, N_{b}=1 \otimes R_{b}^{\prime}$.

(viii) $x \in \mathrm{r}_{\infty}\left(M_{a}\right)$.

Proof. We first construct $M_{n}$ and $N_{n}, n=1,2 \cdots$ which satisfy (i), (iii), and (v). Let $\varepsilon_{j}>0, \sum \varepsilon_{j}<\infty, R_{x_{j}}=\mathrm{R}\left(H_{\nu}^{(j)}, R_{\nu}^{(j)}, \Phi_{\nu}^{(j)}\right), \operatorname{Sp}\left(\Phi_{\nu}^{(j)} / R_{\nu}^{(j)}\right)$ $=\left\{\lambda_{j}, 1-\lambda_{j}\right\}, \lambda_{j}=\left(1+x_{j}\right)^{-1}$ and $\Phi_{\nu}^{(j)}$ be cyclic (and separating) for $R_{\nu}^{(j)}$.

Since $R_{x_{1}}$ is type III, $M \sim M \otimes R_{x_{1}}$ implies $M \stackrel{u}{\sim} M \otimes R_{x_{1}}$. Thus we can write

$$
H=H_{r 1} \otimes H_{1}
$$

2) The authors are indebted to Dr. D. J. C. Bures for pointing out a loophole in an earlier version. For the rest of the paper, the reader can skip this lemma. 


$$
\begin{aligned}
& M=M_{r 1} \otimes M_{(1)} \\
& M^{\prime}=M_{r 1}^{\prime} \otimes M_{(1)}^{\prime}
\end{aligned}
$$

where $M_{r 1} \stackrel{u}{\sim} M, H_{1}=\otimes H_{\nu}^{(1)}, M_{(1)}=R_{x_{1}}$. Choose a unit vector $\psi_{1}$ in $H_{r 1}$ and let

$$
\Phi_{1}=\psi_{1} \otimes \chi_{1}, \chi_{1} \equiv \otimes \Phi_{\nu}^{(1)} .
$$

We set $M_{1}=1 \otimes M_{(1)}, N_{1}=1 \otimes M_{(1)}^{\prime}$.

Since $M_{r 1} \stackrel{u}{\sim} M \stackrel{u}{\sim} M \otimes R_{x 2}$, we have $M_{r 1} \stackrel{u}{\sim} M_{r 1} \otimes R_{x_{2}}$ and we can write

$$
\begin{aligned}
& H_{r 1}=K_{2} \otimes\left(\otimes H_{\nu}^{(2)}\right) \\
& M_{r 1}=R^{(2)} \otimes R_{x 2}
\end{aligned}
$$

where $R^{(2)} \stackrel{u}{\sim} M_{r 1} \stackrel{u}{\sim} M$.

By lemma 2.5, there exists a finite subset $J_{1}$ of indices $\nu$ and a unit vector $\psi_{2}$ of $H_{r 2} \equiv K_{2} \otimes\left(\bigotimes_{\nu \in J_{1}} H_{\nu}^{(2)}\right)$

$$
\begin{aligned}
& \left\|\psi_{2} \otimes \chi_{2}-\psi_{1}\right\|<\varepsilon_{1} \\
& \chi_{2} \equiv \bigotimes_{\nu \leftarrow J_{1}} \Phi_{\nu}^{(2)} .
\end{aligned}
$$

Let

$$
\begin{gathered}
\Phi_{2}=\psi_{2} \otimes \chi_{2} \otimes \chi_{1} \\
H_{2}=\bigotimes_{\nu \mp J_{1}} H_{\nu}^{(2)}, M_{(2)}=\bigotimes_{\nu \mp J_{1}} R_{\nu}^{(2)}, M_{r 2}=R^{(2)} \otimes\left(\bigotimes_{\nu \in J_{1}} R_{\nu}^{(2)}\right) .
\end{gathered}
$$

Then

$$
\begin{aligned}
& H=H_{r 2} \otimes H_{2} \otimes H_{1} \\
& M=M_{r 2} \otimes M_{(2)} \otimes M_{(1)},
\end{aligned}
$$

$M_{r 2} \stackrel{u}{\sim} R^{(2)} \stackrel{u}{\sim} M, \Phi_{2}$ is a product vector and

$$
\left\|\Phi_{2}-\Phi_{1}\right\|<\varepsilon_{1} .
$$

We set

$$
\begin{aligned}
& M_{2}=1 \otimes M_{(2)} \otimes \mathbb{1} \\
& N_{2}=1 \otimes M_{(2)}^{\prime} \otimes 1 .
\end{aligned}
$$

By repetition of this argument we obtain a sequence of Hilbert spaces $H_{j}, H_{r j}$, factors $M_{(j)}, M_{r j}$, and vectors $\chi_{j} \in H_{j}, \Phi_{j} \in H$, such that

$$
H=H_{r j} \otimes\left(\bigotimes_{n=j} H_{n}\right)
$$




$$
\begin{aligned}
& M=M_{r j} \otimes\left(\bigotimes_{n \leq j} M_{(n)}\right) \\
& \Phi_{j}=\psi_{j} \otimes\left(\bigotimes_{n \leq j} \chi_{n}\right) \\
& \left\|\Phi_{j}-\Phi_{j-1}\right\|<\varepsilon_{j-1}
\end{aligned}
$$

where

$$
\begin{aligned}
& H_{n}=\bigotimes_{\nu \neq J_{n-1}}\left(H_{\nu}^{(n)}, \Phi_{\nu}^{(n)}\right) \\
& M_{(n)}=\mathrm{R}\left(H_{\nu}^{(n)}, R_{\nu}^{(n)}, \Phi_{\nu}^{(n)} ; \nu \notin J_{n-1}\right) \sim R_{x_{n}} \\
& \chi_{n}=\bigotimes_{\nu \mp J_{n-1}} \Phi_{\nu}^{(n)} .
\end{aligned}
$$

We then set

$$
\begin{aligned}
& M_{n}=\mathbf{1}_{r j} \otimes M_{(n)} \otimes\left(\bigotimes_{j<n} \mathbf{1}_{j}\right) \\
& N_{n}=\mathbf{1}_{r j} \otimes M_{(n)}^{\prime} \otimes\left(\bigotimes_{j<n} \mathbf{1}_{j}\right)
\end{aligned}
$$

which satisfies (i) by construction.

Since

$$
\left\|\Phi_{j+k}-\Phi_{j}\right\|<\sum_{i=j}^{j+k-1} \varepsilon_{i} \rightarrow 0(j \rightarrow \infty)
$$

$\left\{\Phi_{j}\right\}$ is a Cauchy sequence. Let

$$
\chi=\lim _{j \rightarrow \infty} \Phi_{j} .
$$

By construction $\|\chi\|=\left\|\Phi_{j}\right\|=1$ and

$$
Q_{n} \equiv\left(M_{n} \cup N_{n}\right)^{\prime \prime}
$$

is a type $\mathrm{I}$ factor. Let the minimal projection of $Q_{n}$ defined by $\chi_{n}$ be $E_{n}$. Then $E_{n} \Phi_{j}=\Phi_{j}$ for $j \geq n$ and hence $E_{n} \chi=\chi$. Namely, $\chi=\chi_{r n} \otimes\left(\bigotimes_{j \leq n} \chi_{j}\right)$ and $Q_{n}$ is irreducible on

$$
\begin{aligned}
& \overline{Q_{n} \chi}=\chi_{r n} \otimes H_{n} \otimes\left(\bigotimes_{j<n} \chi_{j}\right) \\
& =\overline{M_{n} \chi}=\overline{N_{n} \chi} .
\end{aligned}
$$

From this, it follows $\left(\prod_{n \leq N} M_{n}\right) \chi$ and $\left(\prod_{n \leq N} N_{n}\right) \chi$ span the same space $H^{(N)}$ $\equiv \chi_{r}^{N} \otimes\left(\otimes_{n \leq N} H_{n}\right)$ and hence (iii) follows. Furthermore, $\chi$ considered as a state on $M_{n}, N_{n}, n=1,2, \cdots$ is the same as the product state of $\chi_{j}$ and hence $M_{a}, N_{a}$ and $\chi$ restricted to $H_{a}$ is unitarily equivalent to $\mathrm{R}\left(H_{n}\right.$, $\left.M_{(n)}, \chi_{n}\right), \mathrm{R}\left(H_{n}, M_{(n)}^{\prime}, \chi_{n}\right)$ and $\otimes \chi_{n}$ on $\otimes H_{n}$. This proves (v). 
Now we proceed to the other conditions. Let $E$ be the projection on $\overline{M^{\prime} \chi}$. By (iii), $\overline{M_{a} M^{\prime} \chi}=\overline{M^{\prime} \chi}$ and hence $E$ commutes with both $M_{a}$ and $N_{a}$. Since $M$ is a factor of type III and $E \in M, M \sim M_{E}$ and we consider $M_{E}$ on $\mathrm{EH}$ instead of $M$ on $H$. Then $\left(M_{n}\right)_{E},\left(N_{n}\right)_{E}$ and $E \chi$ $=\chi$ replace the rôle of $M_{n}, N_{n}$ and $\chi$. Obviously (i), (iii) and (v) are satisfied. In addition, $\chi$ is now cyclic for $M^{\prime}$ and hence separating for $M$. Henceforth, we drop ()$_{E}$. A similar procedure using $\overline{M \chi}$ makes $\chi$ cyclic for $M$ without losing its cyclicity for $M^{\prime}$.

If $\chi$ is separating for $M$, then $M_{a} \mid H_{a} \sim M_{a}$ and hence $M_{a} \sim \bigotimes_{n} R_{x_{n}}$. Similarly $N_{a} \sim \bigotimes_{n} R_{x_{n}}$. Thus (iv) and (ii) are proved. (viii) is then immediate.

We consider $R_{\nu}^{(n)}$ in $M_{(n)}=\otimes R_{\nu}^{(n)}$ and denote the corresponding type I ${ }_{2}$ factor in $M_{n}$ by $M_{n \nu}$. Similarly we write $N_{n \nu}$ for $R_{\nu}^{(n)}$. We now proceed to (vi) and (vii). Let $u_{i j}^{n \nu}$ and $v_{i j}^{n \nu}$ be standard matrix units of $M_{n \nu}$ and $N_{n \nu}$ relative to $\Phi_{\nu}^{(n)}$. Let $l(n, \nu)$ be a one-to-one map of $(n, \nu)$ to the natural numbers and let

$$
\begin{aligned}
& \tau_{n \nu}^{i j} A=\sum_{k} u_{k t}^{n v} A u_{j k}^{n \nu} \\
& \tau_{n \nu} A=\lambda_{1} \tau_{n \nu}^{11} A+\left(1-\lambda_{1}\right) \tau_{n \nu}^{22} A \\
& \tau_{N}^{L} A=\left(\prod_{N\langle(n, \nu) \leq L} \tau_{n \nu}\right) A .
\end{aligned}
$$

We now verify the following properties for $A \in M$.

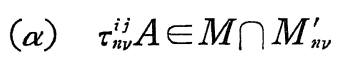

(B) $\left\|\tau_{n v} A\right\| \leqq\|A\|$

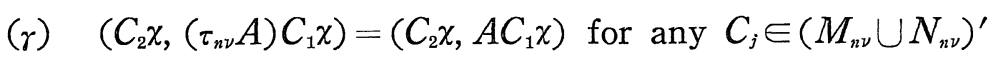

(ס) If $A \in M_{n v}^{\prime}$, then $\tau_{n v} A=A$.

The property $(\alpha)$ is easily checked by calculating $\left[u_{i j}^{n \nu}, \tau_{n \nu}^{k l} A\right] . \quad(\beta)$ follows from

$$
\begin{aligned}
\left\|\sum_{k} u_{k i}^{n} A u_{j k}^{n v} \psi\right\|^{2} & =\sum_{k}\left\|u_{k i}^{n \nu} A u_{j k}^{n v} \psi\right\|^{2} . \\
& \leqq \sum_{k}\left\|A u_{j k}^{n \nu} \psi\right\|^{2} \\
& \leqq\|A\|^{2} \sum_{k}\left\|u_{j k}^{n \nu} \psi\right\|^{2}=\|A\|^{2}\|\psi\|^{2} .
\end{aligned}
$$

(r) follows from $\lambda_{j}^{1 / 2} u_{j k}^{n \nu} \chi=\lambda_{k}^{1 / 2} v_{k j}^{n \nu} \chi,\left[C_{j}, u_{i k}^{n \nu}\right]=\left[C_{j}, v_{k i}^{n \nu}\right]=\left[A, v_{k i}^{n \nu}\right]=0, \sum_{i} v_{i k}^{n \nu} v_{k i}^{n \nu}$ 
$=1$ and $\sum \lambda_{k}=1 . \quad(\delta)$ follows from $\sum_{k} u_{k i}^{n \nu} u_{i k}^{n \nu}=1$.

Let $\tau_{N} A$ be any operator in $\bigcap_{G}\left(\bigcup_{L>G} \tau_{N}^{L} A\right)^{\mathrm{w}}$, where $\mathrm{w}$ denotes the weak closure. Since $\tau_{N}^{L} A$ is bounded by $(\beta)$, this set is non-empty by the weak compactness. By $(\alpha),(\beta),(\gamma)$ and $(\delta)$, we have

$\left(\alpha^{\prime}\right) \quad \tau_{L} A \in M \cap M_{n \nu}^{\prime}$ for all $l(n, \nu)>L$

$\left(\beta^{\prime}\right) \quad\left\|\tau_{L} A\right\| \leq\|A\|$

$\left(\gamma^{\prime}\right) \quad\left(C_{2} \chi,\left(\tau_{L} A\right) C_{1} \chi\right)=\left(C_{2} \chi, A C_{1} \chi\right)$

$\left(\delta^{\prime}\right)$ If $A \in M_{a}^{\prime}, \tau_{L} A=A$.

$$
\text { for any } C_{j} \in Q^{(L)} \equiv \bigcap_{l(n \nu)>L}\left(M_{n \nu} \cup N_{n \nu}\right)^{\prime}
$$

For each sequence $I=\left\{i_{n \nu}, j_{n \nu}\right\}, l(n, \nu) \leq L$, let

$$
\tau_{L}(I) A=\left(\prod_{l(n \nu) \leq L} \tau_{n \nu}^{i_{n \nu} j_{n \nu}}\right)\left(\tau_{L} A\right)
$$

Then

$$
\tau_{L} A=\sum_{I}\left(\prod_{(n \nu) \leq L} u_{i_{n \nu} j_{n \nu}}^{n \nu}\right) \tau_{L}(I) A
$$

because $\sum_{i j} u_{i j}^{n \nu} \tau_{n \nu}^{i j} A=\sum_{i j} u_{i i}^{n \nu} A u_{j j}^{n \nu}=A$. Furthermore $\tau_{L}(I) A \in M_{b}$ by $\left(\alpha^{\prime}\right)$ and $(\alpha)$. Hence $\tau_{L} A \in M_{0}$.

Let $\mathrm{P}$ be the projection on the subspace spanned by $\cup_{L} Q^{(L)} \chi$. Then $\left(\gamma^{\prime}\right)$ implies that $M_{0}=P M P$ on $P H$. Exactly the same argument can

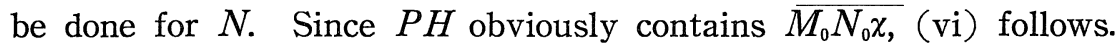

Since $\chi$ is separating for $M$ and $N$, we have only to prove (vii) on restrictions of the relevant algebra to the state given by $\chi$. Since $\chi$ gives a product state for $\left(M_{b} \cup N_{b}\right)^{\prime \prime},\left(M_{n \nu} \cup N_{n \nu}\right)^{\prime \prime}, n,=1,2, \cdots$, we have

$$
\begin{array}{ll}
M_{0}=R_{a} \otimes R_{b}, & N_{0}=R_{a}^{\prime} \otimes S_{b}, \\
M_{a}=R_{a} \otimes 1, & M_{b}=\mathbf{1} \otimes R_{b} \\
N_{a}=R_{a}^{\prime} \otimes 1, & N_{b}=1 \otimes S_{b} .
\end{array}
$$

We now prcve that $M_{0}^{\prime}=N_{0}$ on $\overline{M_{0} N_{0} \chi}$, which proves $S_{b}=R_{b}^{\prime}$.

Let $F$ be any projection in the commutant of $M_{0}$ on $\overline{M_{0} N_{0} \chi}$. Let $F_{1}$ be the projection on $\overline{M F H}$. Since 


$$
M F H=M_{0} F H+M_{c} F H=F H+M_{c} F H
$$

where $M_{c} F H \perp \overline{M_{0} N_{0} \chi}$. Furthermore, $F_{1} \in M^{\prime}$. Hence, by (vi), we have $F \in N_{0}$.

Q.E. D.

The following lemma will be used in lemma 6.11 where it is needed only for the case that all $\mathfrak{U}_{\nu}$ are type I factors. For the case where all $\mathfrak{A}_{\nu}$ except one are type I factors, it has been proved by Araki ( $[3]$, lemma 5). We give here a stronger lemma, based on the result of Tomita [19] that $(\mathfrak{A} \otimes \mathfrak{B})^{\prime}=\mathfrak{Y}^{\prime} \otimes \mathfrak{B}^{\prime}$. If either $\mathfrak{A}$ or $\mathfrak{B}$ is a finite type I factor then one can easily prove that $(\mathfrak{A} \otimes \mathfrak{B})^{\prime}=\mathfrak{H}^{\prime} \otimes \mathfrak{B}^{\prime}$. The following proof then provides an alternate proof of lemma 5 of [3] (without recourse to Tomita's general result), because a type $I_{\infty}$ factor is an ITPFI factor.

Lemma 6.10. Given the ITPS $H=\bigotimes_{\nu=1}^{\infty}\left(H_{\nu}, \Omega_{\nu}\right)$ and von Neumann algebras $\mathfrak{A}_{\nu} \subset \mathscr{B}\left(H_{\nu}\right)$. Then $\left(\otimes \mathfrak{N}_{\nu}\right)^{\prime}=\otimes \mathfrak{N}_{\nu}^{\prime}$.

Proof. Let $\mathfrak{A}=\otimes \mathfrak{A}_{\nu}$. Clearly $\mathfrak{H}^{\prime} \supset \otimes \mathfrak{H}_{\nu}^{\prime}$. For any $T \in \mathfrak{H}^{\prime}$ and any finite index set $J$ define $T_{J} \in \mathcal{B}(H(J))$ by

$$
\left(\psi_{1}^{J}, T_{J} \psi_{2}^{J}\right)=\left(\psi_{1}^{J} \otimes \Omega\left(J^{\mathrm{c}}\right), T \psi_{2}^{J} \otimes \Omega\left(J^{\mathrm{c}}\right)\right) .
$$

Then

$$
\left\|T_{J}\right\| \leq\|T\|
$$

and

$$
T_{J} \in\left(\bigotimes_{\nu \in J} \mathfrak{A}_{\nu}\right)^{\prime}=\bigotimes_{\nu \in J} \mathfrak{X}_{\nu}^{\prime}
$$

Now let

$$
N=\left\{S:\left|\left(\Phi_{j}^{1},(S-T) \Phi_{j}^{2}\right)\right|<\varepsilon, j=1, \cdots n\right\}
$$

be a weak neighbourhood of $T \in \mathfrak{A}^{\prime}$. We construct $S \in N, S \in \otimes \mathfrak{R}_{\nu}^{\prime}$ as follows. We can assume $\left\|\Phi_{j}^{i}\right\| \leq 1, j=1, \cdots n, i=1,2$. By lemma 2.7 there exists a finite set $J$ and vectors $\psi_{j}^{i} \in H(J),\left\|\psi_{j}^{i}\right\| \leq 1$ such that

$$
\left\|\psi_{j}^{i} \otimes \Omega\left(J^{c}\right)-\Phi_{j}^{i}\right\|<(1 / 4) \varepsilon\|T\|^{-1} .
$$

Then for any $S$ we have

$$
\begin{gathered}
\left|\left(\Phi_{j}^{1},(S-T) \Phi_{j}^{2}\right)-\left(\psi_{j}^{1} \otimes \Omega\left(J^{\mathrm{c}}\right),(S-T) \psi_{j}^{2} \otimes \Omega\left(J^{\mathrm{c}}\right)\right)\right| \\
<(1 / 2) \varepsilon(\|S\|+\|T\|)\|T\|^{-1} .
\end{gathered}
$$


It follows that $S=T_{J} \otimes 1$ gives the desired $S$. Thus we have $\mathfrak{Y}^{\prime} \subset \otimes \mathfrak{Y}_{\nu}^{\prime}$.

Q.E.D.

Lemma 6.11. If $M$ has a cyclic and separating vector, then $\mathrm{r}_{\infty}(M)=\mathrm{r}_{\infty}\left(M^{\prime}\right)$.

Proof. Since $M, R_{x}$ have cyclic and separating vectors so does $M \otimes R_{x}$, thus $M \sim M \otimes R_{x}$ if and only if $M \stackrel{u}{\sim} M \otimes R_{x}$, and similarly for $M^{\prime}$. Using lemma 6.10 and $R_{x}^{\prime} \stackrel{u}{\sim} R_{x}$ it follows that $M \stackrel{u}{\sim} M \otimes R_{x}$ if and only if $M^{\prime} \stackrel{u}{\sim} M^{\prime} \otimes R_{x}$.

Q. E. D.

\section{Classification of ITPFI Factors-The Class $\mathbf{S}_{\infty}$}

In this section we prove that all ITPFI factors in the class $S_{\infty}$ are isomorphic. This result is obtained by generalizing lemma 5.4 to arbitrary $N_{\alpha}$. For this purpose we introduce the notion of an $\left(x_{2}, \cdots x_{p}\right)$ sequence.

Definition 7.1. $\left(I_{n}, K_{n}^{1}, K_{n}^{2}, \cdots K_{n}^{p} ; \phi_{n}^{2} \cdots \phi_{n}^{p}\right), n=1,2, \cdots$ is called an $\left(x_{2}, \cdots x_{p}\right)$ sequence for $\mathrm{R}\left(M_{\nu}, \Omega_{\nu}\right)$ if $K_{n}^{1}, \cdots K_{n}^{p}$ are mutually disjoint subsets of $\operatorname{Sp}\left(\Omega\left(I_{n}\right) / M\left(I_{n}\right)\right)$ and $\left(I_{n}, K_{n}^{1}, K_{n}^{j}, \phi_{n}^{j}\right), j=2, \cdots p$ is an $x_{j}$ sequence for $\mathrm{R}\left(M_{\nu}, \Omega_{\nu}\right)$.

Lemma 7.2. Given $\mathrm{R}\left(M_{\nu}, \Omega_{\nu}\right)$ and $x_{2}, \cdots x_{p} \in \mathrm{r}_{\infty}(M, \Omega)$. Then there exists an $\left(x_{2}, \cdots x_{p}\right)$-sequence for $\mathrm{R}\left(M_{\nu}, \Omega_{\nu}\right)$.

Proof. Let $\left(\bar{I}_{j k}, \bar{K}_{j k}^{i}, \bar{\phi}_{j k}\right), k \in I_{\infty}$ be $x_{j}$-sequences, $j=2, \cdots p$. By corollary 3.4 we can assume that $\lambda\left(\bar{K}_{j k}^{1}\right)>1 / 2\left(1+x_{j}\right)^{-1}$ for all $j, k$. We can inductively choose subsequences $k(j, n), n=1,2, \cdots$ such that $\bar{I}_{j, k(j, n)}$ are mutually disjoint. Define $I_{j_{n}}={\overline{I_{j, k(j, n)}}}_{\text {and let }}$

$$
I_{n}=\bigcup_{j=2}^{p} I_{j n} .
$$

For all $\lambda \in \operatorname{Sp}\left(\Omega\left(I_{n}\right) / M\left(I_{n}\right)\right)$ we have

$$
\lambda=\prod_{j=2}^{p} \lambda(j), \lambda(j) \in \operatorname{Sp}\left(\Omega\left(I_{j_{n}}\right) / M\left(I_{j_{n}}\right)\right) .
$$

Define

$$
K_{n}^{1}=\left\{\lambda \in \operatorname{Sp} \Omega\left(I_{n}\right): \lambda(j) \in K_{j n}^{1} \text { for all } j=2, \cdots p\right\}
$$




$$
\begin{aligned}
\left(\phi_{n}^{j} \lambda\right)\left(j^{\prime}\right) & =\left\{\begin{array}{l}
\lambda\left(j^{\prime}\right) \text { if } j^{\prime} \neq j \\
\phi_{j_{n}} \lambda(j) \text { if } j^{\prime}=j
\end{array}\right. \\
K_{n}^{j} & =\phi_{n}^{j} K_{n}^{1}
\end{aligned}
$$

for $j=2, \cdots, p$. By construction, $\left(I_{n}, K_{n}^{1}, K_{n}^{j}, \phi_{n}^{j}\right)$ satisfies Eq. (3.3) for each $j=2, \cdots p$. We have

$$
\lambda\left(K_{n}^{1}\right)=\prod_{j=2}^{p} \lambda\left(K_{j n}^{1}\right)>2^{-p} \prod_{j=2}^{p}\left(1+x_{j}\right)^{-1}>0
$$

which implies $\sum \lambda\left(K_{n}^{1}\right)=\infty$.

The following three lemmas are straightforward generalizations of lemmas 3.3, 5.1, and 5.4 respectively.

Lemma 7.3. Given $\mathrm{R}\left(M_{\nu}, \Omega_{\nu}\right), x_{2} \cdots x_{p} \in \mathrm{r}_{\infty}(M, \Omega)$, and $\varepsilon_{n}>0$. Then there exists an $\left(x_{2}, \cdots x_{p}\right)$-sequence $\left(I_{n}, K_{n}^{1}, K_{n}^{j}, \phi_{n}^{j} ; j=2, \cdots p\right)$ satisfying

$$
\left|1-\sum_{i=1}^{p} \lambda\left(K_{n}^{i}\right)\right|<\varepsilon_{n} .
$$

Proof. The proof is essentially identical to that for lemma 3.3 with $L_{n}^{i}, K_{m}^{i}, i=1,2$ replaced by $L_{n}^{i}, K_{m}^{i}, i=1, \cdots p$ and $\psi_{n}, \phi_{m}$ replaced by $\psi_{n}^{j}, \phi_{m}^{j}, j=2, \cdots p$.

Q.E. D.

Lemma 7.4. Given $M=\mathrm{R}\left(H_{\nu}, M_{\nu}, \Omega_{\nu} ; \nu \in A\right), \lambda_{1} \geq \lambda_{2} \geq \cdots \geq \lambda_{p} \geq 0$, $\sum_{i=1}^{p} \lambda_{i}=1$, and $1>\varepsilon>0$. Let $x_{j}=\lambda_{j} / \lambda_{1}, j=2, \cdots p$. If $x_{j} \in \mathrm{r}_{\infty}(M, \Omega)$ for all $j$, then there is a finite subset $I \subset A$, projections $P, P^{\prime}$ in $M(I)$, $M(I)^{\prime}$ respectively, a unit vector $\Phi \in P P^{\prime} H(I)$, and a type $I_{p}$ factor $N \subset M(I)_{P P^{\prime}}$ such that $\left\|\left(1-P P^{\prime}\right) \Omega(I)\right\|<\varepsilon,\left\|\Phi-P P^{\prime} \Omega(I)\right\|<\varepsilon, \operatorname{Sp}(\Phi / N)$ $=\left(\lambda_{1}, \cdots \lambda_{p}\right)$, and $\Phi$ factorizes $N$ in $M(I)_{P P^{\prime}}$.

Proof. Define

$$
\begin{aligned}
& \varepsilon^{\prime}=\min \left\{\varepsilon /\left(c_{p}+p\right), x_{j} \neq 0\right\} \\
& \varepsilon_{j}^{\prime \prime}=\left\{\begin{array}{l}
x_{j}^{1 / 2} \varepsilon^{\prime} \text { if } x_{j} \neq 0 \\
\varepsilon^{\prime 2} \text { if } x_{j}=0 .
\end{array}\right.
\end{aligned}
$$

By lemma 7.3 there exists a finite $I \subset A$, disjoint sets $K^{1}, \cdots$ $K^{p} \subset \operatorname{Sp}(\Omega(I) / M(I))$ and bijections $\phi^{j}$ from $K^{1}$ to $K^{j}, j=2, \cdots p$ satisfying

$$
\max _{\mu \in K^{1}}\left|x_{j}-\phi^{j} \mu / \mu\right|<\varepsilon_{j}^{\prime \prime}, j=2, \cdots p
$$


and

$$
\left|1-\sum_{i=1}^{p} \lambda\left(K^{i}\right)\right|<\varepsilon /\left(c_{p}+p\right)
$$

where $c_{p}$ is given by lemma 4.2. Eq. (7.10) implies that

$$
\max _{\mu \in K^{1}}\left|x_{j}^{1 / 2}-\left(\phi^{j} \mu / \mu\right)^{1 / 2}\right|<\varepsilon^{\prime}
$$

The result now follows from lemma 4.2.

Q. E. D.

Lemma 7.5. Given $M=\mathrm{R}\left(M_{\nu}, \Omega_{\nu}\right), N=\mathrm{R}\left(N_{\alpha}, \psi_{\alpha}\right)$. If $\hat{\mathrm{r}}\left(N, \psi^{\prime}\right) \subset$ $\mathrm{r}_{\infty}(M, \Omega)$ then $M \sim M \otimes N$.

Proof. The proof is essentially identical to that of lemma 5.4. (with lemma 5.1 replaced by lemma 7.4).

Q. E. D.

Theorem 7.6. Given ITPFI factors $M=\mathrm{R}\left(M_{\nu}, \Omega_{\nu}\right), N=\mathrm{R}\left(N_{\alpha}, \psi_{\alpha}\right)$. If $\mathrm{r}_{\infty}(M)=\mathrm{r}_{\infty}(N)=S_{\infty}$, then $M \sim N$.

Proof. For any ITPFI factor $M=\mathrm{R}\left(M_{\nu}, \Omega_{\nu}\right)$ we have $\hat{\mathrm{r}}(M, \Omega) \subset S_{\infty}$. Thus it follows from lemma 7.5 that $\mathrm{r}_{\infty}(N)=S_{\infty}$ implies $N \sim N \otimes M$. Conversely $\mathrm{r}_{\infty}(M)=S_{\infty}$ implies $M \sim M \otimes N$, and thus $M \sim N$. Q. E. D.

Definition 7.7. We shall denote the ITPFI factor $M$ with $\mathrm{r}_{\infty}(M)$ $=S_{\infty}$ by $R_{\infty}$.

\section{Some Technical Lemmas}

This section contains a number of technical lemmas which are devoted to proving the result that for ITPFI factors $M, \mathrm{r}_{\infty}(M)=S_{x}$ if and only if $M \sim R_{x}$ (theorem 9.1). The basic idea is to exploit the condition for unitary equivalence given by lemma 2.13. The main results of this section are given in lemmas $8.3,8.11,8.14,8.16$.

Definition 8.1. Given a type $I_{n}$ factor $M$, a unit vector $\Omega$, $\operatorname{Sp}(\Omega / M)=\left\{\lambda_{1}, \cdots \lambda_{n}\right\}$. We define

$$
\begin{aligned}
& \delta_{0}(M, \Omega)=\left(\lambda_{1}^{1 / 2}-1\right)^{2}+\sum_{j=2}^{n} \lambda_{j} \\
& \delta_{1}(M, \Omega)=\sum_{j=1}^{n}\left(\lambda_{j}^{1 / 2}-n^{-1 / 2}\right)^{2}
\end{aligned}
$$


and

$$
\delta_{x}(M, \Omega)=\min _{\left(m_{1}, \cdots m_{n}\right)} \sum_{j=1}^{n}\left[\lambda_{j}^{1 / 2}-\left(x^{m_{j}} / \sum_{i=1}^{n} x^{m_{j}}\right)^{1 / 2}\right]^{2} \quad 0<x<1
$$

where the minimum is taken over all $n$-tuples of integers $\left(m_{1}, \cdots m_{n}\right)$.

Note that the expression on the right-hand side of Eq. (8.1) does not change when $m_{j} \rightarrow m_{j}+m$. Thus the $n$-tuple of integers $\left(m_{1}, \cdots m_{n}\right)$ which gives the minimum in Eq. (8.1) is determined only up to an additive integer. This ambiguity could be removed by requiring that $m_{1}=0$, but this is unnecessary for our purposes.

Definition 8.2. Given $0 \leq x \leq 1, M=\mathrm{R}\left(M_{\nu}, \Omega_{\nu}\right)$. We define

$$
\mathrm{d}_{x}(M, \Omega)=\sum_{\nu} \delta_{x}\left(M_{\nu}, \Omega_{\nu}\right)
$$

where $\delta_{x}\left(M_{\nu}, \Omega_{\nu}\right)$ is given by definition 8.1 .

Lemma 8.3. Given $0 \leq x \leq 1, M=\mathrm{R}\left(M_{\nu}, \Omega_{\nu}\right), x \in \mathrm{r}_{\infty}(M), \mathrm{d}_{x}(M, \Omega)$ $<\infty$. Then $M \sim R_{x}$.

Proof. Let $M_{1}=\mathrm{R}\left(M_{\nu}, \Omega_{\nu}^{\prime}\right)$ where $\operatorname{Sp}\left(\Omega_{\nu}^{\prime} / M_{\nu}\right)=\left\{\alpha_{\nu^{\prime}} ; j=1, \cdots n_{\nu}\right\}$ and $\alpha_{\nu j}$ are defined as follows. If $x=0$ let $\alpha_{\nu 1}=1, \alpha_{\nu j}=0, j=2, \cdots n_{\nu}$. If $x=1$ let $\alpha_{\nu j}=n_{\nu}^{-1}, j=1, \cdots n_{\nu}$. If $0<x<1$ let

$$
\alpha_{\nu j}=x^{m_{\nu j}} / \sum_{i=1}^{n_{\nu}} x^{m_{\nu i}}
$$

where $\left(m_{\nu 1}, \cdots m_{\nu n_{\nu}}\right)$ gives the minimum for $\delta_{x}\left(M_{\nu}, \Omega_{\nu}\right)$ in Eq. (8.1). By construction $\hat{\mathrm{r}}\left(M_{\nu}, \Omega_{\nu}^{\prime}\right) \subset S_{x}$ and thus $R_{x} \sim R_{x} \otimes M_{1}$ by lemma 7.5. By lemma 2.13, $\mathrm{d}_{x}(M, \Omega)<\infty$ implies that $M \stackrel{u}{\sim} M_{1}$. Thus $x \in \mathrm{r}_{\infty}\left(M_{1}\right)$ and $M_{1} \sim M_{1} \otimes R_{x}$ by corollary 5.5. Thus $M \sim M_{1} \sim M_{1} \otimes R_{x} \sim R_{x}$.

$$
\text { Q. E. D. }
$$

The following lemmas are devoted to proving the converse result, namely that $\mathrm{d}_{x}(M, \Omega)=\infty$ implies that $\mathrm{r}_{\infty}(M) \neq S_{x}$. The basic idea is to use the central limit theorem to obtain the existence of some $y \in \mathrm{r}_{\infty}(M), y \notin S_{x}$ (except for $x=0$ where we use lemma 3.8).

Lemma 8.4. Given $X_{\nu}=\left\{\lambda_{\nu 1}, \cdots \lambda_{\nu_{\nu}}\right\}, \lambda_{\nu_{i}} \geq 0$ and $\sum_{i=1}^{n_{\nu}} \lambda_{\nu_{i}}=1$. Let $\mu_{\nu}$ be the probability measure on $X_{\nu}$ defined by $\mu_{\nu}(\{\lambda\})=\lambda$, $\lambda \in X_{\nu}$. Let $K_{\nu}^{1}, K_{\nu}^{2}$ be disjoint subsets of $X_{\nu}$ and let $\phi_{\nu}$ be a bijection from $K_{\nu}^{1}$ to $K_{\nu}^{2}$. Assume $0 \notin K_{\nu}^{i}$. Let 


$$
\eta(\lambda)=\log \left(\phi_{\nu} \lambda / \lambda\right), \quad \lambda \in K_{\nu}^{1} .
$$

Let $s_{\nu}$ be the random variable defined by

$$
s_{\nu}(\lambda)= \begin{cases}\eta(\lambda) & \text { if } \lambda \in K_{\nu}^{1} \\ -\eta\left(\phi^{-1} \lambda\right) & \text { if } \lambda \in K_{\nu}^{2} \\ 0 & \text { if } \lambda \notin K_{\nu}^{1} \cup K_{\nu}^{2} .\end{cases}
$$

Let

$$
Y_{N}=\sum_{\nu=1}^{N} s_{\nu}
$$

and

$$
\delta_{\nu}=\max _{\lambda \in K \downarrow}|\eta(\lambda)| .
$$

If

$$
\lim _{\nu \rightarrow \infty} \delta_{\nu}=0
$$

and

$$
\sum_{\nu} \sum_{\lambda \in K_{i}} \lambda_{\eta}(\lambda)^{2}=\infty
$$

then for any fixed $0<a<\infty$ we have

$$
\lim _{N \rightarrow \infty}\left(\prod_{\nu=1}^{N} \mu_{\nu}\right)\left(X_{N}(a)\right)=0
$$

where $X_{N}(a)$ is the subset of $\prod_{\nu=1}^{N} X_{\nu}$ defined by $\left|Y_{N}\right| \leq a$.

Proof. The mean of $s_{\nu}$ is

$$
\begin{aligned}
\left\langle S_{\nu}\right\rangle & =\sum_{\lambda \in K_{\nu}} \eta_{\nu}(\lambda)\left(\lambda-\phi_{\nu} \lambda\right)=\sum \lambda \eta_{\nu}(\lambda)\left(1-e^{\eta_{\nu}(\lambda)}\right) \\
& =-\sum \lambda \eta_{\nu}(\lambda)^{2}\left[1+0\left(\delta_{\nu}\right)\right] .
\end{aligned}
$$

Since $\sum_{\lambda \in K_{\nu}^{\prime}} \lambda \leq 1$ and $\left|\eta_{\nu}(\lambda)\right| \leq \delta_{\nu}$ we have

$$
\left\langle s_{\nu}\right\rangle=0\left(\delta_{\nu}^{2}\right) .
$$

Thus $\left\langle s_{\nu}\right\rangle$ is bounded. The variance of $s_{v}$ is

$$
\begin{aligned}
\sigma_{\nu} & =\sum_{\lambda \in K_{\nu}^{1}}\left[\lambda \eta_{\nu}(\lambda)^{2}+\left(\phi_{\nu} \lambda\right) \eta_{\nu}(\lambda)^{2}\right]-\left\langle s_{\nu}\right\rangle^{2} \\
& =\sum_{\lambda \in K_{\nu}^{\frac{1}{\nu}}} \lambda \eta_{\nu}(\lambda)^{2}\left[1+e^{\eta_{\nu}}\right]-\left\langle s_{\nu}\right\rangle^{2}
\end{aligned}
$$




$$
=2 \sum_{\lambda \in K_{\nu}^{1}} \lambda \eta_{\nu}(\lambda)^{2}\left[1+0\left(\delta_{\nu}\right)\right]-\left\langle s_{\nu}\right\rangle^{2} .
$$

Using $\left\langle s_{\nu}\right\rangle=0\left(\delta_{\nu}^{2}\right)$ for one factor of $\left\langle s_{\nu}\right\rangle^{2}$ and Eq. (8.10) for another, we get

$$
\sigma_{\nu}=2 \sum_{\lambda \in K_{\nu}^{1}} \lambda \eta_{\nu}(\lambda)^{2}\left[1+0\left(\delta_{\nu}\right)\right]
$$

It follows that $\sigma_{\nu} \rightarrow 0$ and $\sum_{\nu} \sigma_{\nu}=\infty$. It now follows from the central limit theorem for bounded variances (Loève [9]) that

$$
\sum_{\nu=1}^{N}\left(s_{\nu}-\left\langle s_{\nu}\right\rangle\right) /\left\{\sum_{\nu=1}^{N} \sigma_{\nu}\right\}^{1 / 2}
$$

approaches a normal distribution as $N \rightarrow \infty$. Since $\sum \sigma_{\nu}=\infty$, the finite interval $[-a, a]$ gives a vanishing contribution as $N \rightarrow \infty$. Q. E. D.

Lemma 8.5. Given $\mathrm{R}\left(M_{\nu}, \Omega_{\nu} ; \nu \in A\right)$. Let $X_{\nu}=\operatorname{Sp}\left(\Omega_{\nu} / M_{\nu}\right)$. Let $K_{\nu}^{1}, K_{\nu}^{2}, \phi_{\nu}, \eta(\lambda), \delta_{\nu}$ be as in lemma 8.4, except $K_{\nu}^{2}$ may contain 0's. If

$$
\lim _{\nu \rightarrow \infty} \delta_{\nu}=0
$$

and

$$
\sum_{\nu} \sum_{\lambda \in K_{\nu}^{1}} \lambda \eta(\lambda)^{2}=\infty
$$

then $\mathrm{r}_{\infty}(M)=S_{\infty}$. Here the terms with $\eta(\lambda)=-\infty$ are excluded from the sum in (8.13).

Proof. We will use lemma 8.4 to prove that $e^{-l} \in \mathrm{r}_{\infty}(M)$ for arbitrary $0<l<\infty$, which implies that $\mathrm{r}_{\infty}(M)=S_{\infty}$. By (8.12), we can restrict $\nu$ such that $0 \notin K_{\nu}^{2}$.

Let $0<l<\infty$ be given. Let $\mu_{\nu}$ be the probability measure and let $s_{\nu}$ be the random variable defined in lemma 8.4. Let $I$ be any finite subset of $A$, and define

$$
Y(I)=\sum_{\nu \in I} s_{\nu}
$$

Let $X(I, a)$ be the subset of $\prod_{\nu \in I} \mathrm{Sp}\left(\Omega_{\nu} / M_{\nu}\right)=\operatorname{Sp}(\Omega(I) / M(I))$ defined by $|Y(I)| \leq a$. It follows from lemma 8.4 that there exist mutually disjoint subsets $I_{n} \subset A, n \in I_{\infty}$ such that 
(8. 15)

$$
\left(\prod_{\nu \in I_{n}} \mu_{\nu}\right)\left(X\left(I_{n}, 1 / 2 l\right)\right)<1 / 2 .
$$

We now construct an $e^{l}$-sequence $\left(I_{n}, L_{n}^{i}, \psi_{n}\right)$ as follows.

For $\rho \in X^{(n)}=\operatorname{Sp}\left(\Omega\left(I_{n}\right) / M\left(I_{n}\right)\right)$ the equation

$$
\rho=\prod_{\nu \in I_{n}} \lambda_{\nu}(\rho), \lambda_{\nu}(\rho) \in \operatorname{Sp}\left(\Omega_{\nu} / M_{\nu}\right)
$$

defines the function $\lambda_{\nu}(\rho), \nu \in I_{n}$. Let

$$
y\left(I_{n}, m, \rho\right)=\sum_{\substack{\nu \leq m \\ \nu \in I_{n}}} s_{\nu}\left(\lambda_{\nu}(\rho)\right)
$$

and define

$$
\alpha\left(I_{n}, \rho\right)=\left\{\begin{array}{l}
\text { minimum } \alpha \in I_{n} \text { with }\left|y\left(I_{n}, \alpha, \rho\right)\right| \geq 1 / 2 l \\
\infty \text { if }\left|y\left(I_{n}, \alpha, \rho\right)\right|<1 / 2 l \text { for all } \alpha \in I
\end{array}\right.
$$

Let

$$
\begin{aligned}
& L_{n}^{1}=\left\{\rho: \alpha\left(I_{n}, \rho\right)<\infty, y\left(I_{n}, \alpha\left(I_{n}, \rho\right), \rho\right) \geq 1 / 2 l, \lambda_{\nu}(\rho) \neq 0\right\} \\
& L_{n}^{2}=\left\{\rho: \alpha\left(I_{n}, \rho\right)<\infty, y\left(I_{n}, \alpha\left(I_{n}, \rho\right), \rho\right) \leq-1 / 2 l, \lambda_{\nu}(\rho) \neq 0\right\} .
\end{aligned}
$$

We define a bijection $\psi_{n}$ from $L_{n}^{1}$ to $L_{n}^{2}$ as follows. If $\nu \leq \alpha\left(I_{n}, \rho\right), \nu \in I_{n}$ define

$$
\lambda_{\nu}\left(\psi_{n} \rho\right)= \begin{cases}\phi_{\nu} \lambda_{\nu}(\rho) & \text { if } \lambda_{\nu}(\rho) \in K_{\nu}^{1} \\ \phi_{\nu}^{-1} \lambda_{\nu}(\rho) & \text { if } \lambda_{\nu}(\rho) \in K_{\nu}^{2} \\ \lambda_{\nu}(\rho) & \text { if } \lambda_{\nu}(\rho) \notin K_{\nu}^{1} \cup K_{\nu}^{2}\end{cases}
$$

where $\phi_{\nu}^{-1}$ is the inverse of the bijection $\phi_{\nu}$. If $\nu>\alpha\left(I_{n}, \rho\right), \nu \in I_{n}$ define

$$
\lambda_{\nu}\left(\psi_{n} \rho\right)=\lambda_{\nu}(\rho) .
$$

Let

$$
\delta\left(I_{n}\right)=\sup _{\nu \in I_{n}} \delta_{\nu}
$$

By construction we have

$$
e^{-l-2 \delta\left(I_{n}\right)}>\psi_{n} \rho / \rho \geq e^{-l}, \rho \in L_{n}^{1} .
$$

Since $\delta\left(I_{n}\right) \rightarrow 0$ we have

$$
\lim _{n \rightarrow \infty} \max _{\rho \in L_{n}^{1}}\left|e^{-l}-\psi_{n} \rho / \rho\right|=0 .
$$

Also by construction we have

$$
1-\lambda\left(L_{n}^{1}\right)-\lambda\left(L_{n}^{2}\right) \leq\left(\prod_{\nu \in I n} \mu_{\nu}\right)\left(X\left(I_{n}, 1 / 2 l\right)\right)<1 / 2 .
$$

It follows from Eqs. (8.24), (8.25) that 


$$
\sum_{n} \lambda\left(L_{n}^{1}\right)=\infty \text {. }
$$

Thus $\left(I_{n}, L_{n}^{i}, \psi_{n}\right)$ is an $e^{-l}$-sequence.

Q.E.D.

Lemma 8.6. Given $0<a<\infty, M=\mathrm{R}\left(M_{\nu}, \Omega_{\nu} ; \nu \in A\right)$, disjoint subsets $K_{\nu}^{1}, K_{\nu}^{2} \subset \operatorname{Sp}\left(\Omega_{\nu} / M_{\nu}\right)$, and a bijection $\phi_{\nu}$ from $K_{\nu}^{1}$ to $K_{\nu}^{2}$. If

$$
\sum_{\nu} \sum_{\lambda \in K_{\nu}^{1}}\left[\lambda^{1 / 2}-\left(\phi_{\nu} \lambda\right)^{1^{12}}\right]^{2}=\infty
$$

then there exists some $x \in \mathrm{r}_{\infty}(M), x \neq 1$. If we also have

$$
\left|\log \left(\phi_{\nu} \lambda / \lambda\right)\right| \leq a
$$

for all $\lambda \in K_{\nu}^{1}$ and all $\nu$, then there exists some $x \in \mathrm{r}_{\infty}(M), e^{-a} \leq x<1$.

Proof. First we throw out from $K_{\nu}^{1}$ and $K_{\nu}^{2}$ all $\lambda$ and $\phi_{\nu} \lambda$ for which $\lambda=\phi_{\nu} \lambda=0$. Since Eqs. (8.26), (8.27) are unaffected by the interchange of $\lambda$ and $\phi_{\nu} \lambda$, we can assume $\phi_{\nu} \lambda \leq \lambda$ and thus $\phi_{\nu} \lambda / \lambda \in[0,1]$ for all $\lambda \in K_{\nu}^{1}$ and all $\nu$. We define a subset $S$ of $[0,1]$ as follows. Let $\varepsilon_{m}>0, \varepsilon_{m} \rightarrow 0, m \in I_{\infty}$. Given $\alpha<\beta$, let

Define

$$
\Sigma(\alpha, \beta)=\sum_{\nu} \sum_{\substack{\lambda \in K_{1}^{1} \\ \phi_{\nu} \lambda / \lambda \in(\alpha, \beta)}}\left[\lambda^{1 / 2}-\left(\phi_{\nu} \lambda\right)^{1 / 2}\right]^{2} .
$$

$$
S=\left\{x: \Sigma\left(x-\varepsilon_{m}, x+\varepsilon_{m}\right)=\infty \text { for all } m\right\} .
$$

We now use the fact that $[0,1]$ is compact to prove that $S$ is nonempty. If $x \notin S$ then there is some finite integer $m(x)$ such that

$$
\Sigma\left(x-\varepsilon_{m(x)}, x+\varepsilon_{m(x)}\right)<\infty .
$$

If $S$ is empty then we have a covering of $[0,1]$ by the open sets $I(x$, $m(x)), x \in[0,1]$. It follows that there is a finite collection $I\left(x_{1}, m\left(x_{1}\right)\right)$, $\cdots I\left(x_{p}, m\left(x_{p}\right)\right)$ which covers $[0,1]$. Using Eqs. (8.30), (8.28) we get

$$
\sum_{\nu} \sum_{\lambda \in K_{\nu}^{1}}\left[\lambda^{1 / 2}-\left(\phi_{\nu} \lambda\right)^{1 / 2}\right]^{2} \leq_{j=1}^{p} \sum\left(x_{j}-\varepsilon_{m\left(x_{j}\right)}, x_{j}+\varepsilon_{m\left(x_{j}\right)}\right)<\infty
$$

which contradicts Eq. (8.26). Thus $S$ is nonempty.

Let $x \in S$. It follows from Eq. (8.29) that we can inductively choose subsets $L_{\nu}^{1}(x) \subset K_{\nu}^{1}$ such that

$$
\lim _{\nu \rightarrow \infty} \max _{\lambda \in L_{\nu}^{1}(x)}\left|x-\phi_{\nu} \lambda / \lambda\right|=0
$$


and

$$
\sum_{\nu} \sum_{\lambda \in L_{\nu}^{1}(x)}\left[\lambda^{1 / 2}-\left(\phi_{\nu} \lambda\right)^{1 / 2}\right]^{2}=\infty
$$

Let

$$
L_{\nu}^{2}(x)=\left\{\phi_{\nu} \lambda: \lambda \in L_{\nu}^{1}(x)\right\} .
$$

If $x \neq 1$ it follows from Eqs. (8.32), (8.33) that

$$
\sum_{\nu} \lambda\left(L_{\nu}^{1}(x)\right)=\infty
$$

It follows from Eqs. (8.32), (8.34) that $\left(\nu, L_{\nu}^{i}(x), \phi_{\nu}\right)$ is an $x$-sequence. If $x=1$, then $\mathrm{R}\left(M_{\nu}, \Omega_{\nu}\right), L_{\nu}^{1}(x), L_{\nu}^{2}(x)$ and $\phi_{\nu}$ satisfy the conditions of lemma 8.5 and we have $\mathrm{r}_{\infty}(M)=S_{\infty}$. If Eq. (8.27) is satisfied then $S \subset\left[e^{-a}, 1\right]$ and thus $x \in \mathrm{r}_{\infty}(M)$ for some $e^{-a} \leq x<1$. Q. E. D.

Lemma 8.7. Given $0<a<\infty, \quad \alpha_{\nu j}>0, \quad-a \leq \eta_{\nu j} \leq a, j=1, \cdots n_{\nu}$, $\nu \in I_{\infty}$. Then the following statements hold.

(i) The conditions

$$
\begin{aligned}
& \sum_{\nu, j} \alpha_{\nu j}\left(e^{\eta_{\nu j} / 2}-1\right)^{2}=\infty \\
& \sum_{\nu, j} \alpha_{\nu j}\left(e^{\eta_{\nu j}}-1\right)^{1}=\infty
\end{aligned}
$$

are equivalent.

(ii) The conditions

$$
\begin{aligned}
& \sum_{\nu} \sum_{i<j} \alpha_{\nu i} \alpha_{\nu j}\left(e^{\eta_{\nu i}}-e^{\eta_{\nu j}}\right)^{2}=\infty \\
& \sum_{\nu} \sum_{i<j} \alpha_{\nu i} \alpha_{\nu j}\left(e^{\eta_{\nu i} / 2}-e^{\eta_{\nu j} / 2}\right)^{2}=\infty \\
& \sum_{\nu} \sum_{i<j} \alpha_{\nu i} \alpha_{\nu j}\left(\eta_{\nu i}-\eta_{\nu j}\right)^{2}=\infty
\end{aligned}
$$

are equivalent.

Proof. Let $f(\eta)=e^{\eta}-1$. For any $\eta \in[-a, a]$ we have

$$
0<f^{\prime}(-a)|\eta|<\left|e^{\eta}-1\right|<f^{\prime}(a)|\eta|<\infty
$$

It follows that for $\eta_{\nu j} \in[-a, a]$, there exist positive constants $C_{1}, C_{2}$ such that

$$
C_{1}\left|e^{\eta_{\nu j} / 2}-1\right| \geq\left|e^{\eta_{\nu j}}-1\right| \geq C_{2}\left|e^{\eta_{\nu j} / 2}-1\right|
$$

from which (i) follows. Statement (ii) follows from a similar argument.

Q. E. D. 
Lemma 8.8. Given $\alpha_{1}, \cdots \alpha_{n}>0, \sum \alpha_{j}=1$, and $-\infty<X_{j}<\infty, j=$ $1, \cdots n$ such that

$$
\sum \alpha_{j} X_{j}=0 .
$$

Then

$$
\sum_{\substack{i, j=1 \\ i<j}}^{n} \alpha_{i} \alpha_{j}\left(X_{i}-X_{j}\right)^{2}=\sum_{i=1}^{n} \alpha_{i} X_{i}^{2} .
$$

Proof. The left hand side of Eq. (8.41) is

$$
(1 / 2) \sum \alpha_{i} \alpha_{j}\left(X_{i}-X_{j}\right)^{2}=\left(\sum \alpha_{i} X_{i}^{2}\right)\left(\sum \alpha_{j}\right)-\left(\sum \alpha_{i} X_{i}\right)^{2} .
$$

By the assumption, this is the same as the right hand side of (8.41).

Lemma 8.9. Given $0<x<1, \lambda_{1}, \cdots \lambda_{n}>0, \sum_{i=1}^{n} \lambda_{i}=1$. Then there exists an $n$-tuple of integers $\left(m_{1}, \cdots m_{n}\right)$ such that

$$
\left|\eta_{i}-\eta_{j}\right|<|\log x|, i, j=1, \cdots n
$$

where $\eta_{j}$ is defined by

$$
\lambda_{j}=e^{\eta_{j}}\left(x^{m_{j}} / \sum_{i=1}^{n} x^{m_{i}}\right), j=1, \cdots n .
$$

Also

$$
\max \left|\eta_{j}\right|<|\log x| .
$$

Proof. For any $-\infty<\alpha<\infty$ we define integers $m_{j}(\alpha), j=1, \cdots n$ by

$$
e^{\alpha} x^{m_{j}(\alpha)-1}>\lambda_{j} \geq e^{\alpha} x^{m_{j}(\alpha)} .
$$

Define $\eta_{j}^{\prime}(\alpha)$ by

$$
\lambda_{j}=e^{\eta_{j}^{\prime}(\alpha)} e^{\alpha} x^{m_{j}(\alpha)} .
$$

It follows from Eqs. (8.45), (8.46) that

$$
0 \leq \eta_{j}^{\prime}(\alpha)<|\log x| \text {. }
$$

Define $\eta_{j}(\alpha)$ by Eq. (8.43) with $m_{j}=m_{j}(\alpha)$. Then we have

$$
\eta_{j}(\alpha)=\eta_{j}^{\prime}(\alpha)-\eta(\alpha)
$$

where

$$
\eta(\alpha)=-\log \left(\sum_{j} x^{m_{j}(\alpha)}\right)-\alpha .
$$


Eq. (8.42) now follows from Eqs. (8.47), (8.48). Using Eq. (8.45) and $\sum \lambda_{j}=1$ we get

$$
x<e^{-\eta(\alpha)} \leq 1 .
$$

Eq. (8.44) now follows from Eqs. (8.47), (8.48), (8.49). Thus $m_{j}$ $=m_{j}(\alpha)$ for any $\alpha$ satisfies the lemma.

Q.E. D.

Lemma 8.10. Given $0<x<1, \lambda_{1}, \cdots \lambda_{n}>0, \sum \lambda_{i}=1$ and $\alpha_{1}, \cdots \alpha_{n}>0$. Then there exists an $n$-tuple of integers $\left(m_{1}, \cdots m_{n}\right)$ satisfying the conditions of lemma 8.9 and a subset $I$ of $\{1, \cdots n\}$ such that

$$
\sup _{i, j \in I}\left|\eta_{i}-\eta_{j}\right| \leq(4 / 5)|\log x|
$$

and

$$
\sum_{\substack{i, j \in I \\ i<j}} \alpha_{i} \alpha_{j}\left(\eta_{i}-\eta_{j}\right)^{2}>(1 / 9) \sum_{\substack{i, j=1 \\ i<j}}^{n} \alpha_{i} \alpha_{j}\left(\eta_{i}-\eta_{j}\right)^{2}
$$

where $\eta_{j}$ is defined by Eq. (8.43).

Proof. Consider the $m_{j}(\alpha), \eta_{j}(\alpha)$ given in the proof of lemma 8.9. We will show that one can choose $\alpha$ and $I$ so that Eqs. (8.50), (8.51) are satisfied.

It follows from Eq. (8.48) that it is sufficient to prove Eqs. (8.50), (8.51) with $\eta_{j}(\alpha)$ replaced by $\eta_{j}^{\prime}(\alpha)$. It follows from Eqs. (8.45), (8.46) that

$$
\eta_{j}^{\prime}(\alpha+\beta)=\eta_{j}^{\prime}(\alpha)-\beta(\operatorname{modulo}|\log x|) .
$$

Thus we can consider the $\eta_{j}^{\prime}(\alpha)$ as defined on a circle of circumference $|\log x|$. Choose the interval $\Delta\left(\eta_{\alpha}\right)=\left[\eta_{\alpha}, \eta_{\alpha}+(1 / 5)|\log x|\right)$ on this circle such that $\sum_{n_{j}^{\prime} \in \Delta\left(\eta_{\alpha}\right)} \alpha_{j}$ is a minimum. We choose $\alpha=\eta_{0}$. Then $\eta_{\alpha} \equiv \eta_{0}-\alpha$ is 0 and this interval is $[0,(1 / 5)|\log x|)$. Let

$$
\Delta_{k}=\left\{j: \eta_{j}^{\prime}(\alpha) \in[(k-1)|\log x| / 5, k|\log x| / 5)\right\}, k=1, \cdots 5
$$

and define

$$
\Sigma_{k l}=\sum_{\substack{i<j \\ i \in \Delta_{k} \\ i \in \Delta_{l}}} \alpha_{i} \alpha_{j}\left(\eta_{i}^{\prime}-\eta_{j}^{\prime}\right)^{2} .
$$

By construction we have 
(8. 55)

$$
\sum_{i \in \Delta_{1}} \alpha_{i} \leqslant \sum_{i \in \Delta_{k}} \alpha_{i}, \quad k=2,3,4,5 .
$$

Now $i_{1} \in \Delta_{1}, i_{2} \in \Delta_{2}, i_{5} \in \Delta_{5}$ implies that

$$
\left|\eta_{i_{1}}^{\prime}-\eta_{i_{5}}^{\prime}\right|<2\left|\eta_{i_{2}}^{\prime}-\eta_{i_{5}}^{\prime}\right| \text {. }
$$

It follows from Eqs. (8.55), (8.56) that

$$
\Sigma_{15}<4 \Sigma_{25}
$$

By similar arguments we have

$$
\begin{aligned}
& \Sigma_{14}<9 \Sigma_{24} \\
& \Sigma_{13}<9 \Sigma_{35} \\
& \Sigma_{12}<\Sigma_{25} \\
& \Sigma_{11}<\Sigma_{25} .
\end{aligned}
$$

Let $I=\bigcup_{k=2}^{5} \Delta_{k}$. It follows from Eq. (8.53) that Eq. (8.50) is satisfied. We have

$$
\sum_{\substack{i, j=1 \\ i<j}}^{n} \alpha_{i} \alpha_{j}\left(\eta_{i}-\eta_{j}\right)^{2}=\sum_{k, l=1}^{5} \sum_{k l}
$$

and

$$
\sum_{\substack{i, j \in I \\ i<j}} \alpha_{i} \alpha_{j}\left(\eta_{i}-\eta_{j}\right)^{2}=\sum_{k, l=2}^{5} \sum_{k l} .
$$

It follows from Eqs. (8.57-63) that Eq. (8.51) is satisfied. Q. E. D.

Lemma 8.11. Given $0<x<1, M=\mathrm{R}\left(M_{\nu}, \Omega_{\nu}\right), \mathrm{r}_{\infty}(M)=S_{x}$. Then $\mathrm{d}_{x}(M, \Omega)<\infty$.

Proof. We will use lemmas $8.7,8.8$ and 8.10 to translate the condition $\mathrm{d}_{x}(M, \Omega)=\infty$ into the conditions of lemma 8.6 with $\mid \log \left(\phi_{\nu} \lambda\right.$ $\mid \lambda)|\leq a<| \log x \mid$. It will then follow that there exists some $y \in \mathrm{r}_{\infty}(M)$, $x<y<1$ which contradicts $\mathrm{r}_{\infty}(M)=S_{x}$.

Let $\operatorname{Sp}\left(\Omega_{\nu} / M_{\nu}\right)=\left\{\lambda_{\nu 1}, \cdots \lambda_{\nu_{\nu}}\right\}$. By lemma 3.15 we can assume $\lambda_{\nu j}>0$ for all $\nu$ and $j$. Use lemma 8.10 to choose integers $\left(m_{\nu 1}, \cdots m_{\nu n_{\nu}}\right)$ and subsets $I_{\nu} \subset\left\{1, \cdots n_{\nu}\right\}$ such that $\left|\eta_{\nu i}\right|<|\log x|$,

$$
\sup _{i, i \in I_{\nu}}\left|\eta_{\nu_{i}}-\eta_{\nu j}\right|<(4 / 5)|\log x|
$$

and 
(8. 65)

$$
\sum_{\substack{i, j \in I_{\nu} \\ i<j}} \alpha_{\nu i} \alpha_{\nu j}\left(\eta_{\nu i}-\eta_{\nu j}\right)^{2}>(1 / 9) \sum_{\substack{i, j=1 \\ i<j}}^{n_{\nu}} \alpha_{\nu i} \alpha_{\nu j}\left(\eta_{\nu i}-\eta_{\nu j}\right)^{2}
$$

where $\eta_{\nu j}$ is defined by

$$
\lambda_{\nu j}=e^{\eta_{\nu j}} \alpha_{\nu j}
$$

and

$$
\alpha_{\nu j}=x^{m_{\nu j}} / \sum_{i=1}^{n_{\nu}} x^{m_{\nu i}}
$$

Let $M_{1}=\mathrm{R}\left(M_{\nu}, \Omega_{\nu}^{\prime}\right)$ where $\operatorname{Sp}\left(\Omega_{\nu}^{\prime} / M_{\nu}\right)=\left\{\alpha_{\nu 1}, \cdots \alpha_{\nu_{\nu}}\right\}$. By Eq. (8.67) $\hat{\mathrm{r}}\left(M_{\nu}, \Omega_{\nu}^{\prime}\right) \subset S_{x}=\mathrm{r}_{\infty}(M)$ and thus $M \sim M \otimes M_{1}$ by lemma 7.5. We have $M \otimes M_{1}=\mathrm{R}\left(M_{\nu} \otimes M_{\nu}, \Omega_{\nu} \otimes \Omega_{\nu}^{\prime}\right)$ where $\operatorname{Sp}\left(\Omega_{\nu} \otimes \Omega_{\nu}^{\prime} / M_{\nu} \otimes M_{\nu}\right)=\left\{\lambda_{\nu i} \alpha_{\nu j}: i, j\right.$ $\left.=1, \cdots n_{\nu}\right\}$. We define disjoint subsets $K_{\nu}^{1}, K_{\nu}^{2} \subset \operatorname{Sp}\left(\Omega_{\nu} \otimes \Omega_{\nu}^{\prime} / M_{\nu} \otimes M_{\nu}\right)$ and a bijection $\phi_{\nu}$ from $K_{\nu}^{1}$ to $K_{\nu}^{2}$ by

$$
\begin{aligned}
& K_{\nu}^{1}=\left\{\lambda_{\nu i} \alpha_{\nu j}: i, j \in I_{\nu} \text { and } i<j\right\} \\
& K_{\nu}^{2}=\left\{\lambda_{\nu i} \alpha_{\nu j}: i, j \in I_{\nu} \text { and } i>j\right\} \\
& \phi_{\nu} \lambda_{\nu i} \alpha_{\nu j}=\lambda_{\nu j} \alpha_{\nu i} \quad(i<j) .
\end{aligned}
$$

For $\lambda=\lambda_{\nu i} \alpha_{\nu j} \in K_{\nu}^{1}$ let

$$
\eta(\lambda)=\log \left(\phi_{\nu} \lambda / \lambda\right)=\eta_{\nu j}-\eta_{\nu i} .
$$

It follows from Eqs. (8.64), (8.71) that

$$
\left|\log \left(\phi_{\nu} \lambda / \lambda\right)\right| \leq(4 / 5)|\log x|
$$

for all $\lambda \in K_{\nu}^{1}$ and all $\nu$. Using Eqs. (8.66), (8.67) and definitions 8.1, 8. 2 the condition $\mathrm{d}_{x}(M, \Omega)=\infty$ implies that

$$
\sum_{\nu} \sum_{j} \alpha_{\nu j}\left(e^{\eta_{\nu} / 2}-1\right)^{2}=\infty
$$

By lemma 8.7 this is equivalent to

$$
\sum \alpha_{\nu j}\left(e^{\eta_{\nu j}}-1\right)^{2}=\infty .
$$

Since $\sum_{j} \lambda_{\nu j}=\sum_{j} \alpha_{\nu j}=1$, it follows from Eq. (8.66) that

$$
\sum \alpha_{\nu j}\left(e^{\eta_{\nu j}}-1\right)=0 \text {. }
$$

Using Eqs. (8.74), (8.75) and lemma 8.8 we get

$$
\sum_{\nu} \sum_{\substack{i, j=1 \\ i<j}}^{n_{\nu}} \alpha_{\nu i} \alpha_{\nu j}\left(e^{\eta_{\nu t}}-e^{\eta_{\nu j}}\right)^{2}=\infty
$$


By lemma 8.7 this is equivalent to

$$
\sum \alpha_{\nu i} \alpha_{\nu j}\left(\eta_{\nu i}-\eta_{\nu j}\right)^{2}=\infty
$$

Eq. (8.65) now gives

$$
\sum_{\substack{\nu, j, j \in I_{\nu} \\ i<j}} \alpha_{\nu i} \alpha_{\nu j}\left(\eta_{\nu i}-\eta_{\nu j}\right)^{2}=\infty .
$$

By lemma 8.7 this is equivalent to

$$
\begin{aligned}
\infty & =\sum_{\substack{i, j \in I_{\nu} \\
i<j}} \alpha_{\nu i} \alpha_{\nu j}\left(e^{\eta_{\nu \downarrow} / 2}-e^{\eta_{\nu j} / 2}\right)^{2} \\
& =\sum_{\nu} \sum_{\lambda \in K_{\nu}^{1}}\left[\lambda^{1 / 2}-\left(\phi_{\nu} \lambda\right)^{1 / 2}\right]^{2} .
\end{aligned}
$$

It follows from Eqs. (8.72), (8.78) and lemma 8.6 that there exists some $e^{l} \in \mathrm{r}_{\infty}(M), 0<|l| \leq(4 / 5)|\log x|$.

Q. E. D.

The result that $\mathrm{r}_{\infty}(M)=S_{x}$ implies that $\mathrm{d}_{x}(M, \Omega)<\infty$ for $x=0,1$ can be obtained directly from the known conditions for $M$ to be type $\mathrm{I}, \mathrm{II}_{1}$ respectively which have been stated in lemma 2.14. However it seems worthwhile to use our techniques to give an independent derivation of these results.

In the proof of lemma 8.11 we made frequent use of the fact that the $\eta_{\nu j}$ were bounded, a condition that does not hold when $x=1$. Instead of modifying the proof of lemma 8.11 , it seems simpler to use the following two lemmas.

Lemma 8.12. Let $x_{j}, j \in K$ be a finite set of real numbers such that

$$
\sum_{j \in K} x_{j}=0 .
$$

Then there exist disjoint subsets $K^{1}, K^{2} \subset K$ and a bijection $\phi$ from $K^{1}$ to $K^{2}$ such that

$$
\sum_{j \in K^{1}}\left(x_{j}-x_{\phi(j)}\right)^{2} \geq \sum_{j \in K} x_{j}^{2} .
$$

Proof. Order the index set $K$ by $x_{1} \geq x_{2} \geq \cdots \geq x_{N}$ and choose $m$ so that either $N=2 m$ or $N=2 m+1$. Let $\alpha=x_{m+1}$. Let $K^{1}=\{1, \cdots m\}$, $K^{2}=\{N-m+1, \cdots N\}$, and define $\phi(j)=N-m+j, j \in K^{1}$. Let

$$
y_{j}=x_{j}-\alpha, j=1, \cdots N \text {. }
$$


By construction, if $j \in K^{1}$ then $y_{j}$ and $y_{\phi(j)}$ have the opposite sign. Thus we have

$$
\begin{aligned}
\sum_{j \in K^{1}}\left(x_{j}-x_{\phi(j)}\right)^{2} & =\sum_{j \in K^{1}}\left(y_{j}-y_{\phi(j)}\right)^{2} . \\
& \geq \sum_{j \in K^{1}}\left(y_{j}^{2}+y_{\phi(j)}^{2}\right)=\sum_{j=1}^{N} y_{j}^{2}
\end{aligned}
$$

where we used the fact that $y_{j}=0$ if $j \notin K^{1} \cup K^{2}$. Since $\sum x_{j}=0$ we have

$$
\sum_{j=1}^{N} y_{j}^{2}=\sum_{j=1}^{N} x_{j}^{2}+N \alpha^{2} \geq \sum x_{j}^{2}
$$

Q. E. D.

Lemma 8.13. Given $\lambda_{1}, \cdots \lambda_{n} \geq 0, \sum \lambda_{i}=1$. Then there exist disjoint subsets $K^{1}, K^{2} \subset\left\{\lambda_{1}, \cdots \lambda_{n}\right\}$ and a bijection $\phi$ from $K^{1}$ to $K^{2}$ such that

$$
\sum_{\lambda \in K^{1}}\left[\lambda^{1 / 2}-(\phi \lambda)^{1 / 2}\right]^{2}>1 / 2 \sum_{j=1}^{n}\left(\lambda_{j}^{1 / 2}-n^{-1 / 2}\right)^{2} .
$$

Proof. Let

$$
\delta_{j}=\lambda_{j}^{1 / 2}-n^{-1 / 2}
$$

Then

$$
\lambda_{j}=\delta_{j}^{2}+n^{-1}+2 n^{-1 / 2} \delta_{j}
$$

and using $\sum \lambda_{j}=1$ we get

$$
\bar{\delta}=n^{-1} \sum \delta_{j}=-1 / 2 n^{-1 / 2} \sum \delta_{j}^{2} .
$$

We also get

$$
\delta_{j}^{2}=\lambda_{j}+n^{-1}-2 \lambda_{j}^{1 / 2} n^{-1 / 2}
$$

and thus
(8. 87)
$\sum \delta_{j}^{2}<2$

Since

(8. 88)

$$
\sum\left(\boldsymbol{\delta}_{j}-\overline{\boldsymbol{\delta}}\right)=0
$$

we have

$$
\sum \delta_{j}^{2}=\sum\left(\delta_{j}-\bar{\delta}\right)^{2}+n \bar{\delta}^{2} .
$$

Using Eqs. (8.86), (8.87) we get 


$$
\sum\left(\delta_{j}-\bar{\delta}\right)^{2}=\sum \delta_{j}^{2}-1 / 4\left(\sum \delta_{j}^{2}\right)^{2}>1 / 2 \sum \delta_{j}^{2} .
$$

Since $\lambda_{i}^{1 / 2}-\lambda_{j}^{1 / 2}=\delta_{i}-\delta_{j}$, the lemma now follows from Eqs. (8. 88), (8. 89) and lemma 8.12.

Q.E. D.

Lemma 8.14. Given $M=\mathrm{R}\left(M_{\nu}, \Omega_{\nu}\right), \mathrm{r}_{\infty}(M)=S_{1}$. Then $\mathrm{d}_{1}(M, \Omega)$ $<\infty$.

Proof. Assume $\mathrm{d}_{1}(M, \Omega)=\infty$. Then we have

$$
\sum_{\nu} \sum_{j=1}^{n_{\nu}}\left(\lambda_{\nu j}^{1 / 2}-n_{\nu}^{-1 / 2}\right)^{2}=\infty
$$

where $\operatorname{Sp}\left(\Omega_{\nu} / M_{\nu}\right)=\left\{\lambda_{\nu 1}, \cdots \lambda_{\nu n_{\nu}}\right\}$. It follows from lemma 8.13 that there exist disjoint subsets $K_{\nu}^{1}, K_{\nu}^{2} \subset \operatorname{Sp}\left(\Omega_{\nu} / M_{\nu}\right)$ and a bijection $\phi_{\nu}$ from $K_{\nu}^{1}$ to $K_{\nu}^{2}$ such that

$$
\sum_{\nu} \sum_{\lambda \in K_{\nu}^{1}}\left[\lambda^{1 / 2}-\left(\phi_{\nu} \lambda\right)^{1 / 2}\right]^{2}=\infty .
$$

It now follows from lemma 8. 6 that there exists some $x \in \mathrm{r}_{\infty}(M), x \neq 1$ which is a contradiction.

Q.E.D.

Finally, we consider the case $x=0$. We first prove

Lemma 8.15. Given $M=\mathrm{R}\left(M_{\nu}, \Omega_{\nu}\right), \operatorname{Sp}\left(\Omega_{\nu} / M_{\nu}\right)=\left\{\lambda_{\nu 1}, \cdots \lambda_{\nu n_{\nu}}\right\}$. The conditions

$$
\begin{aligned}
& \sum_{\nu}\left|1-\lambda_{\nu 1}\right|=\infty \\
& \mathrm{d}_{0}(M, \Omega)=\infty
\end{aligned}
$$

are equivalent, where $\left\|\Omega_{\nu}\right\|=1$.

Proof. We have

$$
\begin{aligned}
\mathrm{d}_{0}(M, \Omega) & =\sum\left[\left(1-\lambda_{\nu 1}^{1 / 2}\right)^{2}+\sum_{j=2}^{n_{\nu}} \lambda_{\nu j}\right] \\
& =\sum\left[1+\lambda_{\nu 1}-2 \lambda_{\nu 1}^{1 / 2}+\left(1-\lambda_{\nu 1}\right)\right] \\
& =2 \sum\left[1-\lambda_{\nu 1}^{1 / 2}\right] .
\end{aligned}
$$

Using $\left(1-\lambda^{1 / 2}\right)^{2} \geq 0$ we obtain the inequality

$$
\left|1-\lambda^{1 / 2}\right| \leq|1-\lambda| \leq 2\left|1-\lambda^{1 / 2}\right|, 0 \leq \lambda \leq 1 .
$$

It follows that 


$$
\mathrm{d}_{0}(M, \Omega) \leq 2 \Sigma\left(1-\lambda_{\nu 1}\right) \leq 2 \mathrm{~d}_{0}(M, \Omega)
$$

Q.E. D.

Lemma 8.16. Given $M=\mathrm{R}\left(M_{\nu}, \Omega_{\nu}\right), \mathrm{r}_{\infty}(M)=S_{0}$. Then $\mathrm{d}_{0}(M, \Omega)$ $<\infty$.

Proof. By lemma $3.8 \mathrm{r}_{\infty}(M)=S_{0}$ if and only if $\sum\left|1-\lambda_{\nu 1}\right|<\infty$. By lemma 8.15 $\Sigma\left|1-\lambda_{\nu 1}\right|<\infty$ if and only if $\mathrm{d}_{0}(M, \Omega)<\infty$. Q. E. D.

All the discussions in this section are valid even if we allow $\left\|\Omega_{\nu}\right\|^{2}$ $=1+\delta_{\nu} \neq 1$. This is due to the following situation. Let $\left(1+\delta_{\nu}\right)=$ $\theta_{\nu}, \lambda_{\nu j}^{\prime}=\theta^{-1} \lambda_{\nu j}, \Omega_{\nu}^{\prime}=\Omega_{\nu} /\left\|\Omega_{\nu}\right\| . \quad$ Then $\operatorname{Sp}\left(\Omega_{\nu}^{\prime} / M_{\nu}\right)=\left\{\lambda_{\nu j}^{\prime}\right\}$ if $\operatorname{Sp}\left(\Omega_{\nu} / M_{\nu}\right)=\left\{\lambda_{\nu j}\right\}$. By Lemma 3.14, $\mathrm{r}_{\infty}\left(M, \Omega^{\prime}\right)=\mathrm{r}_{\infty}(M, \Omega)$. On the other hand, $0<\Pi\left\|\Omega_{\nu}\right\|$ $<\infty$ implies $\sum\left|\delta_{\nu}\right|<\infty$. If $\sum_{j} \alpha_{\nu j}=1$, then

$$
\begin{aligned}
& \sum_{j}\left|\left[\lambda_{\nu j}^{1 / 2}-\alpha_{\nu j}^{1 / 2}\right]^{2}-\left[\lambda_{\nu j}^{1 / 2}-\alpha_{\nu j}^{1 / 2}\right]^{2}\right| \\
\leq & \sum_{j}\left|\lambda_{\nu j}^{1 / 2}-\lambda_{\nu j}^{1 / 2}\right| \cdot\left\{\lambda_{\nu j}^{1 / 2}+\lambda_{\nu j}^{1 / 2}+2 \alpha_{\nu j}^{1 / 2}\right\} \\
\leq & \left\{1+\theta_{\nu}^{1 / 2}+2\right\} \cdot\left|1-\theta_{\nu}^{1 / 2}\right| .
\end{aligned}
$$

Thus if we adopt the Definition 8.1 and 8.2 for $\left\|\Omega_{\nu}\right\| \neq 1$, then $\mathrm{d}_{x}(M, \Omega)$ $=\infty$ and $\mathrm{d}_{x}\left(M, \Omega^{\prime}\right)=\infty$ are equivalent.

\section{Classification of ITPFI Factors-The Classes $\mathbb{S}_{x}$}

In this section we apply the results of the preceding section. We prove that $\mathrm{r}_{\infty}(M)=S_{x}$ if and only if $M \sim R_{x}, 0 \leq x \leq 1$. We obtain some useful criteria for calculating $\mathrm{r}_{\infty}(M)$.

Theorem 9.1. Given $M=\mathrm{R}\left(M_{\nu}, \Omega_{\nu}\right), \mathrm{r}_{\infty}(M)=S_{x}, 0 \leq x \leq 1$. Then $M \sim R_{x}$.

Proof. By lemmas 8.11, 8.14, 8.16 $\mathrm{r}_{\infty}(M)=S_{x}$ implies that $\mathrm{d}_{x}(M, \Omega)<\infty$. By lemma 8.3, $\mathrm{r}_{\infty}(M)=S_{x}$ and $\mathrm{d}_{x}(M, \Omega)<\infty$ implies that $M \sim R_{x}$.

Q. E. D.

We remark that we can prove Theorem 9.1 for $x=1$ without making any use of the condition $\mathrm{d}_{1}(M, \Omega)<\infty$. Namely, by lemma 5.2 $M$ is infinite if and only if $0 \in \mathrm{r}_{\infty}(M)$. Thus $\mathrm{r}_{\infty}(M)=S_{1}$ implies that 
$M$ is finite. Since $1 \in \mathrm{r}_{\infty}(M)$ implies that $M$ cannot be type $\mathrm{I}, M$ must be type $\mathrm{II}_{1}$. Since all hyperfinite type $\mathrm{II}_{1}$ factors are isomorphic (Dixmier [8], Theorem III. 7.1) we have $M \sim R_{1}$.

If $M$ is an ITPFI factor, $\mathrm{r}_{\infty}(M) \neq S_{01}$, then by Theorems 7.6 and 9.1M must be isomorphic to one of the factors $R_{x}, 0 \leq x \leq 1$ or $R_{\infty}$. However, the calculation of $\mathrm{r}_{\infty}(M)$ by a direct application of definition 3.2 may be a nontrivial problem. The following two lemmas give some useful criteria for calculating $\mathrm{r}_{\infty}(M)$. The first lemma is a straightforward variation of lemma 3.5.

Lemma 9.2. Given $M=\mathrm{R}\left(M_{\nu}, \Omega_{\nu}\right), M_{\nu}$ type $\mathrm{I}_{n}$ for all $\nu$, and $\operatorname{Sp}\left(\Omega_{\nu} / M_{\nu}\right)=\left\{\lambda_{1}, \cdots \lambda_{n}\right\}$ independent of $\nu$. Let $\widehat{S}\left(\lambda_{1}, \cdots \lambda_{n}\right)$ be the intersection of all sets $S$ such that

(i) $S$ is one of the sets $S_{x}, 0 \leq x \leq 1, S_{01}$ and $S_{\infty}$

(ii) $\lambda_{j} \neq 0$ implies that $\lambda_{i} / \lambda_{j} \in S, i, j=1, \cdots n$.

Then $\mathrm{r}_{\infty}(M)=\widehat{S}\left(\lambda_{1}, \cdots \lambda_{n}\right)$.

Proof. Since $\widehat{S}\left(\lambda_{1}, \cdots \lambda_{n}\right)$ is nonempty, it must be one of the sets $S_{x}, S_{01}, S_{\infty}$. Thus $\widehat{S}\left(\lambda_{1}, \cdots \lambda_{n}\right)$ is the smallest asymptotic ratio set containing all $\lambda_{i} / \lambda_{j}, \lambda_{j} \neq 0$. It now follows from lemma 3.5 that $\widehat{S}\left(\lambda_{1}, \cdots \lambda_{n}\right)$ $\subset \mathrm{r}_{\infty}(M)$. It follows from definition 3.2 that $\mathrm{r}_{\infty}(M) \subset \widehat{S}\left(\lambda_{1}, \cdots \lambda_{n}\right)$. Q.E. D.

Lemma 9.3. Given $M=\mathrm{R}\left(M_{\nu}, \Omega_{\nu}\right), M_{\nu}$ type $\mathrm{I}_{n}$ for all $\nu, \mathrm{Sp}\left(\Omega_{\nu} / M_{\nu}\right)$ $=\left\{\lambda_{\nu 1}, \cdots \lambda_{\nu n}\right\}$ and

$$
\lim _{\nu \rightarrow \infty} \lambda_{\nu j}=\lambda_{j}, j=1, \cdots n
$$

If

$$
\Delta=\sum_{\nu} \sum_{j=1}^{n}\left(\lambda_{\nu j}^{1 / 2}-\lambda_{j}^{1 / 2}\right)^{2}<\infty
$$

then $\mathrm{r}_{\infty}(M)=\widehat{S}\left(\lambda_{1}, \cdots \lambda_{n}\right)$ (see lemma 9.2 for the definition of $\widehat{S}\left(\lambda_{1}, \cdots\right.$ $\left.\left.\lambda_{n}\right)\right)$. If $\widehat{S}\left(\lambda_{1}, \cdots \lambda_{n}\right)=S_{01}$ in addition then $M \sim R_{0} \otimes R_{1}$. If $\Delta=\infty$ and $\lambda_{j} \neq 0$ for all $j=1, \cdots n$ then $\mathrm{r}_{\infty}(M)=S_{\infty}$.

Proof. By Eq. (9.1) and $\left\|\Omega_{\nu}\right\| \rightarrow 1$, we have $\sum \lambda_{j}=1$. By lemma 
3. 14 we can assume $\sum_{j=1}^{n} \lambda_{\nu j}=1$ (note that this does not affect whether or not $\Delta$ is finite). Let $M_{1}=\mathbb{R}\left(M_{\nu}, \Omega_{\nu}^{\prime}\right)$ where $\operatorname{Sp}\left(\Omega_{\nu}^{\prime} / M_{\nu}\right)=\left\{\lambda_{1}, \cdots \lambda_{n}\right\}$.

Case (i), $\Delta<\infty$. By lemma 2.13 $M \sim M_{1}$. By lemma 9.2, $\mathrm{r}_{\infty}\left(M_{1}\right)$ $=\widehat{S}\left(\lambda_{1}, \cdots \lambda_{n}\right)$. If $\widehat{S}\left(\lambda_{1}, \cdots \lambda_{n}\right)=S_{01}$ then $\hat{\mathrm{r}}\left(M_{1}\right) \subset S_{01}$ and by lemma 7.5 we have $R_{0} \otimes R_{1} \sim R_{0} \otimes R_{1} \otimes M_{1}$ since $\mathrm{r}_{\infty}\left(R_{0} \otimes R_{1}\right)=S_{01}$. Since $0,1 \in \mathrm{r}_{\infty}\left(M_{1}\right)$ we have $M_{1} \sim M_{1} \otimes R_{0}, M_{1} \sim M_{1} \otimes R_{1}$. Thus $M_{1} \sim M_{1} \otimes R_{0} \otimes R_{1} \sim R_{0} \otimes R_{1}$.

Case (ii), $\Delta=\infty$. Consider $M \otimes M_{1}=\mathrm{R}\left(M_{\nu} \otimes M_{\nu}, \Omega_{\nu} \otimes \Omega_{\nu}^{\prime}\right) . \quad$ By lemma $3.5 \hat{\mathrm{r}}\left(M_{\nu}, \Omega_{\nu}^{\prime}\right) \subset \mathrm{r}_{\infty}(M)$ and thus $M \sim M \otimes M_{1}$ by lemma 7.5 . We have $\operatorname{Sp}\left(\Omega_{\nu} \otimes \Omega_{\nu}^{\prime} / M_{\nu} \otimes M_{\nu}\right)=\left\{\lambda_{\nu i} \lambda_{j}: i, j=1, \cdots n\right\}$. Define

$$
K_{\nu}^{1}=\left\{\lambda_{\nu i} \lambda_{j}: i<j\right\}
$$

$$
K_{\nu}^{2}=\left\{\lambda_{\nu i} \lambda_{j}: i>j\right\}
$$

and a bijection $\phi_{\nu}$ from $K_{\nu}^{1}$ to $K_{\nu}^{2}$ by

$$
\phi_{\nu} \lambda_{\nu i} \lambda_{j}=\lambda_{\nu j} \lambda_{i}, i<j .
$$

Define $\eta_{\nu j}$ by

$$
\lambda_{\nu j}=e^{\eta_{\nu j}} \lambda_{j} .
$$

It follows from Eq. (9.1) that

$$
\lim _{\nu \rightarrow \infty} \eta_{\nu j}=0, j=1, \cdots n \text {. }
$$

We have

$$
\infty=\Delta=\sum_{\nu} \sum_{j} \lambda_{j}\left(e^{\eta_{\nu} / 2}-1\right)^{2} .
$$

By lemma 8.7 this is equivalent to

$$
\sum_{\nu} \sum_{j} \lambda_{j}\left(e^{\eta_{\nu j}}-1\right)^{2}=\infty
$$

Since $\sum_{j} \lambda_{\nu j}=\sum_{j} \lambda_{j}=1$ we have

$$
\sum_{j} \lambda_{j}\left(e^{\eta_{\nu}}-1\right)=0
$$

It follows from Eqs. (9.8), (9.9) and lemma 8.8 that

$$
\sum_{\nu} \sum_{i<j} \lambda_{i} \lambda_{j}\left(e^{\eta_{\nu_{i}}}-e^{\eta_{\nu j}}\right)^{2}=\infty .
$$

Using lemma 8.7 again this is equivalent to

$$
\sum_{\nu} \sum_{i<j} \lambda_{i} \lambda_{j}\left(\eta_{\nu i}-\eta_{\nu j}\right)^{2}=\infty
$$


Since $\lambda_{j} \neq 0$ for all $j$, it follows from Eq. (9.1) that this is equivalent to

$$
\sum_{\nu} \sum_{i<j} \lambda_{\nu i} \lambda_{j}\left(\eta_{\nu i}-\eta_{\nu j}\right)^{2}=\infty
$$

For $\lambda=\lambda_{\nu i} \lambda_{j} \in K_{\nu}^{1}$ let

$$
\eta(\lambda)=\log \left(\phi_{\nu} \lambda / \lambda\right)=\eta_{\nu j}-\eta_{\nu i}
$$

It follows from Eqs. (9.7), (9.10), (9.11) that the conditions of lemma 8.5 are satisfied and we have $\mathrm{r}_{\infty}\left(M \otimes M_{1}\right)=S_{\infty}$. Q.E. D.

The statement that $\Delta<\infty, \widehat{S}\left(\lambda_{1}, \cdots \lambda_{n}\right)=S_{01}$ implies $M \sim R_{0} \otimes R_{1}$ is nontrivial since the class $S_{01}$ contains more than one isomorphic class (see Sec. 10). If $\lambda_{j}=0$ for some $j$ then we can have $\Delta=\infty$ but $\mathrm{r}_{\infty}(M)$ $\neq S_{\infty}$ (see lemma 9.4).

If $M$ is an ITPFI factor, $\mathrm{r}_{\infty}(M) \neq S_{01}$, then by theorems $3.9,7.6$ and 9.1 $M$ must be isomorphic to one of the factors $R_{x}, 0 \leq x \leq 1$ or $R_{\infty}$. The factors $R_{x}$ can be obtained as tensor products of type $I_{2}$ factors (see definition 3.10). If $M=\mathrm{R}\left(M_{\nu}, \Omega_{\nu}\right), \operatorname{Sp}\left(\Omega_{\nu} / M_{\nu}\right)=\left(\lambda_{\nu}, 1-\lambda_{\nu}\right)$ and $\lambda_{\nu} \rightarrow 1$, then $0 \in \mathrm{r}_{\infty}(M)$ by lemma 3.5 and thus $M \nsim R_{1}$. However we have

Lemma 9.4. Let $M$ be an ITPFI factor, $\mathrm{r}_{\infty}(M) \neq S_{1}, S_{01}$. Then $M$ can be obtained as $M=\mathrm{R}\left(M_{\nu}, \Omega_{\nu}\right)$ where $M_{\nu}$ is type $\mathrm{I}_{2}, \operatorname{Sp}\left(\Omega_{\nu} / M_{\nu}\right)$ $=\left(\lambda_{\nu}, 1-\lambda_{\nu}\right)$ and $\lambda_{\nu} \rightarrow 1$.

Proof. By theorems 3.9, 7.6 and 9.1 $M$ must be one of the factors $R_{x}, 0 \leq x<1$ or $R_{\infty}, \quad R_{0}$ as given in definition 3.10 is already in the desired form. By lemma 3.13 and theorem 7.6 $R_{x} \otimes R_{y} \propto R_{\infty}$ if $x, y \neq 0,1$ and $\log x / \log y$ is irrational. Thus it remains only to prove the lemma for $R_{x}, 0<x<1$.

Given $0<x<1$, choose integers $N_{j}$ for each $j \in I_{\infty}$ such that $N_{j} x^{2 j}>1$. For each $\nu \in I_{\infty}$ satisfying

$$
2 \sum_{j=1}^{n-1} N_{j}<\nu \leq 2 \sum_{j=1}^{n} N_{j}
$$

let

$$
\lambda_{\nu}= \begin{cases}\left(1+x^{2 n}\right)^{-1} & \text { if } \nu \text { is odd } \\ \left(1+x^{2 n+1}\right)^{-1} & \text { if } \nu \text { is even. }\end{cases}
$$


Consider $M=\mathrm{R}\left(M_{\nu}, \Omega_{\nu}\right)$ where $\operatorname{Sp}\left(\Omega_{\nu} / M_{\nu}\right)=\left(\lambda_{\nu}, 1-\lambda_{\nu}\right)$. Then $\hat{\mathrm{r}}(M, \Omega)$ $\subset S_{x}$ and $R_{x} \sim M \otimes R_{x}$ by lemma 5.4. We construct an $x$-sequence for $\mathrm{R}\left(M_{\nu}, \Omega_{\nu}\right)$ as follows. Let $I_{m}=\{2 m-1,2 m\}, m \in I_{\infty}, K_{m}^{1}=\{(1-$ $\left.\left.\lambda_{2 m-1}\right) \lambda_{2 m}\right\}, K_{n}^{2}=\left\{\lambda_{2 m-1}\left(1-\lambda_{2 m}\right)\right\}$ and let $\phi_{m}$ be the unique bijection from $K_{m}^{1}$ to $K_{m}^{2}$. Then

$$
\phi_{m} \lambda / \lambda=x
$$

for $\lambda \in K_{m}^{1}$ and all $m$. If $\lambda \in K_{m}^{1}$ where $\nu=2 m$ satisfies Eq. (9.12) then we have

$$
\lambda=\left(1+x^{-2 n}\right)^{-1}\left(1+x^{2 n+1}\right)^{-1}>x^{2 n} / 4 .
$$

It follows from Eqs. (9.12), (9.13), (9.15) that

$$
\sum_{m} \lambda\left(K_{m}^{1}\right) \geq \sum_{j} N_{j} x^{2 j} / 4=\infty \text {. }
$$

It follows from Eqs. (9.14), (9.16) that $\left(I_{m}, K_{m}^{i}, \phi_{m}\right)$ is an $x$-sequence. Thus $x \in \mathrm{r}_{\infty}(M)$ and $M \sim M \otimes R_{x} \sim R_{x}$. Q. E. D.

In Sec. 10 we consider ITPFI factors $M=\mathrm{R}\left(M_{\nu}, \Omega_{\nu}\right)$ where $M_{\nu}$ is type $I_{2}$ and $r_{\infty}(M)=S_{01}$. Lemma 10.1 is the analog of lemma 9.4 for these factors. However it is not known whether or not all ITPFI factors in the $S_{01}$ class can be obtained as tensor products of type $\mathrm{I}_{2}$ factors.

\section{The Class $\boldsymbol{S}_{01}$}

In this section we give some elementary properties of tensor products $M=\otimes M_{\nu}$ of type $\mathrm{I}_{2}$ factors $M_{\nu}$ where $\mathrm{r}_{\infty}(M)=S_{01}$. We prove (lemma 10.1) that $M$ is either hyperfinite $I I_{1} \otimes I_{\infty}$, or is type III with $\lambda_{\nu} \rightarrow 1$ where $\operatorname{Sp}\left(\Omega_{\nu} / M_{\nu}\right)=\left(\lambda_{\nu}, 1-\lambda_{\nu}\right)$. We give some conditions that such factors are nonisomorphic. Theorem 10.10 gives explicitly a nondenumerable family of mutually nonisomorphic type III factors with $\mathrm{r}_{\infty}(M)$ $=S_{01}$.

Lemma 10.1. Given $M=\mathrm{R}\left(M_{\nu}, \Omega_{\nu}\right), M_{\nu}$ type $\mathrm{I}_{2}, \operatorname{Sp}\left(\Omega_{\nu} / M_{\nu}\right)=\left(\lambda_{\nu}, 1\right.$ $\left.-\lambda_{\nu}\right), \mathrm{r}_{\infty}(M)=S_{01}$. Then either $M \sim R_{0} \otimes R_{1}$ or $M \sim \mathrm{R}\left(M_{\nu}, \Omega_{\nu}^{\prime}\right), \operatorname{Sp}\left(\Omega_{\nu}^{\prime}\right.$ $\left./ M_{\nu}\right)=\left(\lambda_{\nu}^{\prime}, 1-\lambda_{\nu}^{\prime}\right)$ and $\lambda_{\nu}^{\prime} \rightarrow 1$. In the latter case $M$ is type III. 
Proof. By lemma 3.5, $\mathrm{r}_{\infty}(M)=S_{01}$ implies that the only allowed accumulation points of $\lambda_{\nu}$ are $1 / 2$ and 1 . If 1 is the only accumulation point the first part of the lemma is trivially satisfied. If $1 / 2$ is the only accumulation point, then by lemma $9.3 \mathrm{r}_{\infty}(M)$ is either $S_{1}$ or $S_{\infty}$ which is a contradiction. If $1 / 2$ and 1 are accumulation points then we can write $M=M_{0} \otimes M_{1}$ where $M_{0}, M_{1}$ are tensor products of type $\mathrm{I}_{2}$ factors such that $\lambda_{\nu} \rightarrow 1,1 / 2$ respectively. By lemma 9.3, $r_{\infty}\left(M_{1}\right)$ is either $S_{1}$ or $S_{\infty}$, hence we must have $r_{\infty}\left(M_{1}\right)=S_{1}$ and $M_{1} \sim R_{1}$ by theorem 9.1. $\mathrm{r}_{\infty}\left(M_{0}\right)$ must be either $S_{0}$ or $S_{01}$. If $\mathrm{r}_{\infty}\left(M_{0}\right)=S_{0}$ then $M_{0}$ $\sim R_{0}$ by theorem 9.1 and $M \sim R_{0} \otimes R_{1}$. If $\mathrm{r}_{\infty}\left(M_{0}\right)=S_{01}$ then $M_{0} \sim$ $M_{0} \otimes R_{1}$, hence $M \sim M_{0}$.

If $M \sim \mathbb{R}\left(M_{\nu}, \Omega_{\nu}^{\prime}\right), \operatorname{Sp}\left(\Omega_{\nu}^{\prime} / M_{\nu}\right)=\left(\lambda_{\nu}^{\prime}, 1-\lambda_{\nu}^{\prime}\right)$, where $\lambda_{\nu}^{\prime} \rightarrow 1$ then lemma 3.8 implies that $\sum\left(1-\lambda_{\nu}^{\prime}\right)=\infty$. It then follows from lemma 2.14 that $M$ is type III.

Q. E. D.

Definition 10.2. Given $0 \leq l_{1}<l_{2}<\cdots, l_{j} \rightarrow \infty$ and positive integers $N_{1}, N_{2}, \cdots$. Let

$$
\lambda_{\nu}=\left(1+e^{-l j}\right)^{-1}, N_{1}+\cdots+N_{j-1}<\nu \leq N_{1}+\cdots+N_{j} .
$$

We denote the factor $M=\mathrm{R}\left(M_{\nu}, \Omega_{\nu}\right)$ where $\operatorname{Sp}\left(\Omega_{\nu} / M_{\nu}\right)=\left(\lambda_{\nu}, 1-\lambda_{\nu}\right)$ by $M\left[l_{1}, N_{1} ; l_{2}, N_{2} ; \cdots\right]$ or $M\left[l_{j}, N_{j}\right]$.

Lemma 10.3. Given $M=\mathrm{M}\left[l_{j}, N_{j}\right]$. Then $\mathrm{r}_{\infty}(M)=S_{0}$ if and only if $\sum N_{j} e^{-l j}<\infty$.

Proof. We have $M=\mathrm{R}\left(M_{\nu}, \Omega_{\nu}\right)$ where $\operatorname{Sp}\left(\Omega_{\nu} / M_{\nu}\right)=\left(\lambda_{\nu}, 1-\lambda_{\nu}\right)$. By lemma $3.8 \mathrm{r}_{\infty}(M)=S_{0}$ if and only if $\Sigma\left(1-\lambda_{\nu}\right)<\infty$. Since

$$
1-\lambda_{\nu}=\left(1+e^{l,}\right)^{-1}, N_{1}+\cdots+N_{j-1}<\nu \leq N_{1}+\cdots+N_{j}
$$

we have

$$
\sum_{j} N_{j} e^{-l_{j}}>\sum_{\nu}\left(1-\lambda_{\nu}\right)>1 / 2 \sum N_{j} e^{-l,}
$$

Q.E. D.

Lemma 10.4. Given $M=\mathrm{M}\left[l_{j}, N_{j}\right]$ where $l_{j_{+1}}>\left(N_{j}+1\right) l_{j}$. If $\sum N_{j} e^{-l_{j}}=\infty$ then $\mathrm{r}_{\infty}(M)=S_{01}$.

Proof. Consider any finite set I containing only $\nu>N_{1}+\cdots+N_{j}$. 
Then the interval between different points $\log \mu, \mu \in \operatorname{Sp}(\Omega(I) / M(I))$ is at least $l_{j+1}$. Since $\lim l_{j}=\infty$ it follows that $\mathrm{r}_{\infty}(M) \subset S_{01}$. Since $S_{0}$ is excluded by lemma 10.3 we have $\mathrm{r}_{\infty}(M)=S_{01}$.

Q.E. D.

It seems likely that lemma 10.4 should still hold if the condition $l_{j+1}>\left(N_{j}+1\right) l_{j}$ is replaced by

$$
\lim _{j \rightarrow \infty} l_{j} N_{j}^{1 / 2} / l_{j+1}=0 .
$$

We remark that one can obtain sequences $\left(l_{j}, N_{j}\right)$ satisfying the conditions of lemma 10.4 by choosing $N_{j}$ larger than $e^{l}$ and choosing $l_{j+1}$ larger than $l_{j}\left(N_{j}+1\right)$ for each $j$.

Lemma 10.5. Given $l>0, M=\mathrm{M}\left[n_{j} l, N_{j}\right]$ where the $n_{j}$ are integers. Then $M \otimes R_{x} \sim R_{x}$ where $x=e^{-t}$.

Proof. Clearly $\hat{\mathrm{r}}(M) \subset S_{x}$ and the result follows from lemma 5. 4.

Q.E. D.

Lemma 10.6. Given $0<l, l^{\prime}<\infty, M=\mathrm{R}\left(M_{\nu}, \Omega_{\nu}\right)=\mathrm{M}\left[n_{j} l, N_{j}\right]$ where the $n_{j}$ are integers. Let $x=e^{-l}, x_{k}=e^{-l^{\prime \prime k}}, k \in I_{\infty}$. If $\mathrm{d}_{x_{k}}(M)=\infty$ (see definition 8.2) then $M \otimes R_{x_{1}} \propto R_{x_{k}}$. If this holds for all $k \in I_{\infty}$, then $M \otimes R_{x_{1}} \sim R_{\infty}$. Otherwise let $K$ be the minimum $k$ such that $\mathrm{d}_{x_{k}}(M)$ $<\infty$, then $M \otimes R_{x_{1}} \sim R_{x_{K}}$.

Proof. Since $x_{1} \in \mathrm{r}_{\infty}\left(M \otimes R_{x_{1}}\right)$ it follows that $\mathrm{r}_{\infty}\left(M \otimes R_{x_{1}}\right)$ is either $S_{\infty}$ or $S_{x_{k}}$ for some $k \in I_{\infty}$. By lemma 8.11 $\mathrm{r}_{\infty}\left(M \otimes R_{x_{1}}\right)=S_{x_{k}}$ implies that $d_{x_{k}}\left(M \otimes R_{x_{1}}\right)=\mathrm{d}_{x_{k}}(M)<\infty$. First two conclusions then follows. Conversely, if $\mathrm{d}_{x_{k}}(M)<\infty$ then $M \otimes R_{x_{1}} \otimes R_{x_{k}} \sim R_{x_{k}}$ by lemma 8.3. Hence $\mathrm{r}_{\infty}(M) \subset \mathrm{r}_{\infty}\left(R_{x_{k}}\right)=S_{x_{k}}$. Since $S_{x_{k}} \subset S_{x_{k}}$ implies $k \leq k^{\prime}, \mathrm{r}_{\infty}\left(M \otimes R_{x_{1}}\right)$ $=S_{x_{k}}$ for the minimum $k$ with finite $\mathrm{d}_{x_{k}}(M)$ and hence $M \otimes R_{x_{1}} \sim R_{x_{k}}$.

Corollary 10.7. Given $0<l, l^{\prime}<\infty, M_{1}=\mathrm{M}\left[n_{j} l, N_{j}\right], M_{2}=$ $\mathrm{M}\left[n_{j}^{\prime} l^{\prime}, N_{j}^{\prime}\right] \quad$ where the $n_{j}, n_{j}^{\prime}$ are integers. If $\mathrm{d}_{x}\left(M_{2}\right)=\infty, x=e^{-l}$ then $M_{1} \nsim M_{2}$.

Proof. We have $\mathrm{r}_{\infty}\left(M_{1} \otimes R_{1}\right)=S_{x}$. By lemma 10.6, $\mathrm{d}_{2}\left(M_{2}\right)=\infty$ implies that $\mathrm{r}_{\infty}\left(M_{2} \otimes R_{x}\right) \neq S_{x}$.

Q. E. D.

We remark that given $M_{1}, M_{2}$ as in corollary 10.7 where $l \neq l^{\prime}$, we can obtain $\mathrm{r}_{\infty}\left(M_{1}\right)=\mathrm{r}_{\infty}\left(M_{2}\right)=S_{01}$ and also $\mathrm{d}_{x}\left(M_{2}\right)=\infty$ by taking $N_{j}^{\prime}$ 
sufficiently large for each $j$.

Lemma 10.8. Given $M_{1}=\mathrm{M}\left[l_{j}, N_{j}\right], M_{2}=\mathrm{M}\left[l_{j}^{\prime}, N_{j}\right]$ where $\sum N_{j} e^{-l^{\prime}}$, $=\infty$. If $l_{j}^{\prime}-l_{j} \rightarrow k$ as $j \rightarrow \infty$ then $e^{k} \in \mathrm{r}_{\infty}\left(M_{1} \otimes M_{2}\right)$.

Proof. We have $M_{i}=\mathrm{R}\left(M_{i \nu}, \Omega_{i \nu}\right), \mathrm{i}=1,2$. Let $M_{\nu}=M_{1 \nu} \otimes M_{2 \nu}$, $\Omega_{\nu}=\Omega_{1 \nu} \otimes \Omega_{2 \nu}$. Then $M_{1} \otimes M_{2}=\mathrm{R}\left(M_{\nu}, \Omega_{\nu}\right)$. Let

$$
\begin{aligned}
& \mu_{\nu 1}=\left(1+e^{-l_{j}}\right)^{-1}\left(1+e^{l_{j}^{\prime}}\right)^{-1} \\
& \mu_{\nu 2}=\left(1+e^{l_{j}}\right)^{-1}\left(1+e^{-l^{\prime}}\right)^{-1}=e^{l_{j}^{\prime}-l_{j}} \mu_{\nu 1}
\end{aligned}
$$

where $N_{1}+\cdots+N_{j-1}<\nu \leq N_{1}+\cdots N_{j}$. Then $\mu_{\nu 1}, \mu_{\nu 2} \in \operatorname{Sp}\left(\Omega_{\nu} / M_{\nu}\right)$. Let $I_{\nu}=\{\nu\}, K_{\nu}^{i}=\left\{\mu_{\nu i}\right\}, i=1,2$ and $\phi_{\nu} \mu_{\nu 1}=\mu_{\nu 2}$. We have

$$
\sum_{\nu} \lambda\left(K_{\nu}^{1}\right)=\sum \mu_{\nu 1}>(1 / 4) \sum N_{j} e^{-l_{j}^{\prime}}=\infty
$$

It follows from Eq. (10.6) that

$$
\lim _{\nu \rightarrow \infty}\left|e^{k}-\mu_{\nu 2} / \mu_{\nu 1}\right|=0 .
$$

Thus $\left(I_{\nu}, K_{\nu}^{i}, \phi_{\nu}\right)$ is an $e^{k}$-sequence for $M_{1} \otimes M_{2}$.

Q.E. D.

Corollary 10.9. Given $0<l<\infty, M_{1}=\mathrm{M}\left[n_{j} l, N_{j}\right], M_{2}=\mathrm{M}\left[n_{j}^{\prime} l, N_{j}^{\prime}\right]$ where $n_{j}, n_{j}^{\prime}$ are integers and $\mathrm{r}_{\infty}\left(M_{1}\right)=\mathrm{r}_{\infty}\left(M_{2}\right)=S_{01}$. If

$$
\begin{aligned}
& n_{j+1}>\left(N_{j}^{\prime}+1\right)\left(n_{j}^{\prime}+1\right) \\
& n_{j}^{\prime}+1>\left(N_{j}+1\right) n_{j}
\end{aligned}
$$

then $M_{1} \rtimes M_{2}$.

Proof. Let $M_{3}=M\left[\left(n_{j}^{\prime}+1\right) l, N_{j}^{\prime}\right] . \quad$ By lemma 10.8, $e^{l} \in \mathrm{r}_{\infty}\left(M_{2} \otimes\right.$ $\left.M_{3}\right)$. We can write $M_{1} \otimes M_{3}=\mathrm{M}\left[n_{1} l, N_{1} ;\left(n_{1}^{\prime}+1\right) l, N_{1}^{\prime} ; n_{2} l, N_{2} ; \ldots\right]$. It follows from Eqs. (10.8), (10.9) and lemma 10.4 that $\mathrm{r}_{\infty}\left(M_{1} \otimes M_{3}\right)$ $=S_{01}$.

Q. E. D.

Theorem 10.10. There exist nondenumerably many mutually nonisomorphic factors $M$ with $\mathrm{r}_{\infty}(M)=S_{01}$.

Proof. Let $M_{k}=M\left[l_{j}+k, N_{j}\right], 0 \leq k \leq 1$ where $l_{j+1}>2\left(N_{j}+1\right)\left(l_{j}\right.$ +1 ) and $\sum N_{j} e^{-l_{j}}=\infty$ (this last condition can be achieved by choosing $N_{j}$ sufficiently large for each $\left.j\right)$. By lemma 10.4, $\mathrm{r}_{\infty}\left(M_{k}\right)=\mathrm{r}_{\infty}\left(M_{k} \otimes M_{k}\right)$ $=S_{01}$. By lemma 10.8, $e^{k-k^{\prime}} \in \mathrm{r}_{\infty}\left(M_{k} \otimes M_{k^{\prime}}\right)$. Thus $k \neq k^{\prime}$ implies that 
$M_{k} \propto M_{k^{\prime}}$.

Q.E. D.

\section{Another Algebraic Invariant for ITPFI Factors}

In this section we define a second algebraic invariant $\rho(M)$ for ITPFI factors $M$, and use it to analyze further the $S_{01}$ class.

Definition 11.1. Let $M$ be an ITPFI factor. We define the algebraic invariant $\rho(M)$ as the set of all $x \in[0,1]$ such that $R_{x} \sim R_{x} \otimes M$.

Given $M=\mathrm{R}\left(H_{\nu}, M_{\nu}, \Omega_{\nu}\right)$ we note that $\mathrm{d}_{x}(M, \Omega)$ as given in definition 8.2 does depend on the vector $\Omega=\otimes \Omega_{\nu}$. However if $\psi_{r}=\otimes \psi_{\nu} \in \otimes\left(H_{\nu}, \Omega_{\nu}\right)$ then $\mathrm{d}_{x}(M, \Omega)=\infty$ if and only if $\mathrm{d}_{x}(M, \psi)=\infty$. Thus by a slight abuse of notation we can write $\mathrm{d}_{x}(M)=\infty$ if $\mathrm{d}_{x}(M, \psi r)=\infty$ for any (and thus all) $\psi_{r}=\otimes \psi_{\nu} \in \otimes\left(H_{\nu}, \Omega_{\nu}\right)$.

Lemma 11.2. Given $M=\mathrm{R}\left(M_{\nu}, \Omega_{\nu}\right)$. Then

$$
\rho(M)=\left\{x \in[0,1]: \mathrm{d}_{x}(M)<\infty\right\} .
$$

Proof. Assume $\mathrm{d}_{x}(M)<\infty$. Since $\mathrm{d}_{x}\left(R_{x}\right)<\infty$ it follows that $\mathrm{d}_{x}\left(M \otimes R_{x}\right)<\infty$. Since $x \in \mathrm{r}_{\infty}\left(M \otimes R_{x}\right)$ we have $R_{x} \sim R_{x} \otimes M$ by lemma 8. 3. Conversely, by lemmas $8.11,8.14,8.16 R_{x} \sim R_{x} \otimes M$ implies that $\mathrm{d}_{x}\left(M \otimes R_{x}\right)<\infty$ and thus $\mathrm{d}_{x}(M)<\infty$.

Q.E.D.

Lemma 11.3. Given $0<x<1, M=\mathrm{R}\left(M_{\nu}, \Omega_{\nu}\right), x \in \rho(M)$. Then $x^{1 / n} \in \rho(M), n \in I_{\infty}$.

Proof. By definitions 8.1 and $8.2, \mathrm{~d}_{x^{1 / n}}(M, \Omega) \leq \mathrm{d}_{x}(M, \Omega)$. The result now follows from lemma 11.2.

Q.E.D.

Lemma 11.4. Given ITPFI factors $M, N$. Then

$$
\rho(M \otimes N)=\rho(M) \cap \rho(N) .
$$

Proof. We have $\mathrm{d}_{x}(M \otimes N)<\infty$ if and only if $\mathrm{d}_{x}(M)<\infty$ and $\mathrm{d}_{x}(N)<\infty$. The result now follows from lemma 11.2. Q.E.D.

Lemma 11.5. $\rho\left(R_{0}\right)=[0,1)$

$$
\begin{aligned}
\rho\left(R_{1}\right) & =(0,1] \\
\rho\left(R_{0} \otimes R_{1}\right) & =(0,1)
\end{aligned}
$$




$$
\begin{aligned}
& \rho\left(R_{x}\right)=\left\{x^{1 / n} ; n \in I_{\infty}\right\} \quad 0<x<1 \\
& \rho\left(R_{\infty}\right)=\phi .
\end{aligned}
$$

Proof. We have $x \in \rho\left(R_{y}\right)$ if and only if $R_{x} \sim R_{x} \otimes R_{y}$ which is the case if and only if $y \in r_{\infty}\left(R_{x}\right)=S_{x}$. This argument gives $\rho\left(R_{y}\right)$, $0 \leq y \leq 1$. $\quad \rho\left(R_{0} \otimes R_{1}\right)$ now follows from lemma 11.4. Since $R_{x} \otimes R_{\infty} \sim$ $R_{\infty} \times R_{x}$ for any $x \in[0,1]$ we have $\rho\left(R_{\infty}\right)=\phi$ Q.E.D.

Lemma 11.6. Let $M$ be an ITPFI factor. Then $0 \in \rho(M)$ if and only if $M \sim R_{0}$, and $1 \in \rho(M)$ if and only if $M \sim R_{1}$.

Proof. By lemma 11.5, $0 \in \rho\left(R_{0}\right)$. Conversely, if $0 \in \rho(M)$ then $R_{0} \sim R_{0} \otimes M$ and it follows that $M$ must be type $I$ since $R_{0}$ is type $I_{\infty}$. Since the definition of an ITPFI factor excludes finite type I, we have $M \sim R_{0}$.

By lemma 11.5, $1 \in \rho\left(R_{1}\right)$. Conversely. if $1 \in \rho(M)$ then $R_{1} \sim R_{1}$ $\otimes M$ and it follows that $M$ must be finite since $R_{1}$ is type $\mathrm{II}_{1}$. Since $M$ cannot be finite type $\mathrm{I}$, and all hyperfinite $\mathrm{II}_{1}$ factors are isomorphic, we have $M \sim R_{1}$.

In the remainder of this section we consider tensor products $M=\otimes M_{\nu}$ of type $\mathrm{I}_{2}$ factors $M_{\nu}$.

Lemma 11. 7. Given $0<l, k<\infty, M=\mathrm{R}\left(M_{\nu}, \Omega_{\nu}\right)=\mathrm{M}\left[n_{j} l, N_{j}\right]$ where the $n_{j}$ are integers (see definition 10.2). For each $j$ choose an integer $p_{j}$ so that $\left|\delta_{j}\right|$ is a minimum where

$$
\delta_{j}=p_{j} k-n_{j} l .
$$

Let $y=e^{-k}$. Then $d_{y}(M)<\infty$ if and only if

$$
\sum_{j=1}^{\infty} N_{j} e^{-n_{j} l} \delta_{j}^{2}<\infty
$$

Proof. For each $j$, choose $m_{j}$ so that $\left(0, m_{j}\right)$ gives the minimum for $\delta_{y}\left(M_{\nu}, \Omega_{\nu}\right)$ in Eq. (8.1) where $\sum_{1}^{j-1} N_{i}<\nu \leq \sum_{1}^{j} N_{i}$. Let

$$
\delta_{j}^{\prime}=m_{j} k-n_{j} l \text {. }
$$

Since $1<\left(1+e^{m_{j} k}\right) /\left(1+e^{\left(m_{j}+1\right) k}\right)<e^{k}$ and a similar inequality holds for $-k,\left|\eta_{j}^{\prime}\right|$ is bounded by $k$. Hence we have

$$
\delta_{y}\left(M_{\nu}, \Omega_{\nu}\right)=\left[\left(1+e^{-n_{j} l}\right)^{-1 / 2}-\left(1+e^{-n_{j} l-\delta_{j}^{\prime}}\right)^{-1 / 2}\right]^{2}
$$




$$
\begin{aligned}
& +\left[\left(1+e^{n_{j} l}\right)^{-1 / 2}-\left(1+e^{n_{j} l+\delta^{\prime}}\right)^{-1 / 2}\right]^{2} \\
= & e^{-n_{j} l}\left(1-e^{-\delta_{j}^{\prime} / 2}\right)^{2}\left(1+O\left[e^{-n_{j} l}\right]\right)
\end{aligned}
$$

where the second term is $\left(1+e^{n_{j} l}\right)^{-1}\left(1+e^{n_{j} l} e^{\delta_{\jmath}^{\prime}}\right)^{-1} e^{2 n_{l} l}\left(e^{\delta_{j}^{\prime}}-1\right)^{2}[(1+$ $\left.\left.e^{n, l} e^{\delta \prime}\right)^{1 / 2}+\left(1+e^{n, l}\right)^{1 / 2}\right]^{-2}$ and yields the main contribution. Thus

$$
\mathrm{d}_{y}\left(M_{\nu}, \Omega_{\nu}\right)=\sum_{\nu} \delta_{y}\left(M_{\nu}, \Omega_{\nu}\right)=\sum_{j} N_{j} e^{-n, l}\left(1-e^{\left.\delta^{\prime}\right)^{2}}\left[1+0\left(e^{-n, l}\right)\right]\right.
$$

Since $n_{j} \rightarrow \infty$ (see definition 10.2) it follows that $\mathrm{d}_{y}(M)=\infty$ if and only if

$$
\sum_{j} N_{j} e^{-n, l}\left(1-e^{-\delta^{\prime} / 2}\right)^{2}=\infty
$$

Since $\left|\delta_{j}^{\prime}\right| \leq k$ it follows from the same argument used to prove lemma 8. 7 that Eq. (11.6) is equivalent to

$$
\sum_{j} N_{j} e^{-n_{j} l}\left(\delta_{j}^{\prime}\right)^{2}=\infty
$$

Since $n_{j} \rightarrow \infty$, it follows from definition 8.1 and Eqs. (11.1), (11.3), (11.4) that there is some finite $J$ and some fixed $\varepsilon>0$ such that for all $j>J$, if either $\delta_{j}<\varepsilon$ or $\delta_{j}^{\prime}<\varepsilon$ then $m_{j}=p_{j}$ and $\delta_{j}=\delta_{j}^{\prime}$. Since we also have $\left|\delta_{j}\right|,\left|\delta_{j}^{\prime}\right| \leq \frac{1}{2} k$, it follows that there exist positive constants $C_{1}, C_{2}$ such that

$$
C_{1}\left|\delta_{j}\right| \geq\left|\delta_{j}^{\prime}\right| \geq C_{2}\left|\delta_{j}\right|, \quad j>J
$$

It follows from Eq. (11.8) that Eq. (11.7) is equivalent to Eq. (11.2).

Q.E.D.

Lemma 11.8. Given $l, k_{1}, \cdots k_{n} \in(0, \infty)$ such that $k_{i} / l$ is irrational, $i=1, \cdots n$. Then there exists an ITPFI factor $M$ such that $e^{-j l} \in \rho(M), j \in \mathrm{I}_{\infty}$ and $e^{-k_{t}} \in \rho(M), i=1, \cdots n$.

Proof. Consider $M=\mathrm{R}\left(M_{\nu}, \Omega_{\nu}\right)=\mathrm{M}\left[(j !) l, N_{j}\right]$ where we choose $N_{j}$ as follows. Define

$$
\varepsilon_{j i}=\min _{m_{i}}\left|m_{i} k_{i}-(j !) l\right|, i=1, \cdots n
$$

where the minimum is taken over integers $m_{i}$. Since $k_{i} / l$ is irrational we have $\varepsilon_{j i}>0$ and it follows that we can choose $N_{j}$ sufficiently large 
for each $j$ such that

$$
\sum N_{j} e^{-(j !)} \varepsilon_{j i}^{2}=\infty, i=1, \cdots n .
$$

It follows from lemma 11.7 that $e^{-k_{i}} \notin \rho(M), i=1, \cdots n$. By construction we have

$$
\delta_{e^{-\jmath}}\left(M_{\nu}, \Omega_{\nu}\right)=0 \quad \text { if } \quad \nu>\sum_{i=1}^{j-1} N_{i}
$$

and thus

$$
\mathrm{d}_{e^{-\jmath}}(M, \Omega)=\sum_{\nu} \delta_{e^{-\jmath}}\left(M_{\nu}, \Omega_{\nu}\right)<\infty
$$

for all $j \in \mathrm{I}_{\infty}$. It follows from lemma 11.2 that $e^{-j l} \in \rho(M), j \in \mathrm{I}_{\infty}$.

$$
\text { Q.E.D. }
$$

Corollary 11.9. The ITPFI factors constructed in lemma 11.8 belong to the class $S_{01}$.

Proof. The algebraic invariant $\rho(M)$ is not one of the sets given in lemma 11. 5 .

Q.E.D.

We note that since the $\varepsilon_{j i}$ defined by Eq. (11.9) are bounded, it follows from Eq. (11.10) that

$$
\sum N_{j} e^{-(j !) l}=\infty .
$$

If the condition given in lemma 10.4 were satisfied we would have $N_{j}<j$, which contradicts Eq. (11.12). Furthermore, since Eq. (11.10) is the only condition the $N_{j}$ must satisfy, they can be made arbitrarily large. Thus Eq. (10.4) is not a necessary condition that $\mathrm{r}_{\infty}(M)=$ $S_{01}$.

We now use lemma 11.7 and some results from number theory concerning the approximation of irrationals by rationals to construct more examples of ITPFI factors $M$ in the class $S_{01}$. Given $0<k$, $l<\infty$ and an integer $n$. Choose an integer $m$ such that $\delta=|m k-n l|$ is a minimum. We have

$$
\delta=n k|(l / k)-(m / n)|
$$

We recall that a real number $\xi$ is said to be approximable by rationals to order $p$ if there exists a positive constant $c$ depending 
only on $\xi$ such that the inequality

$$
|\xi-m / n|<c / n^{p}
$$

has infinitely many rational solutions $m / n$ with $n>0$. It is known that all irrational numbers are approximable to order 2 , and that irrational number $\xi$ whose continued fraction has bounded partial quotients cannot be approximated to any order higher than 2 . The set of all irrationals with bounded partial quotients has measure zero, but it has the cardinal number of the continuum. It is an easy matter to construct irrational numbers which can be approximated to any degree $p \geq 2$.

Lemma 11. 10. Let $\xi^{-1}$ be a positive irrational number which is approximable by rationals to order $p=2+\varepsilon, \varepsilon>0$. Given $0<l<\infty$ there exists an ITPFI factor $M$ such that $e^{-l}, e^{-\xi l} \in \rho(M)$ and $e^{-\theta l} \oplus \rho(M)$ where $\theta^{-1}$ is any irrational with bounded partial quotients.

Proof. There is a positive constant $c$ and an infinite sequence of integers $m_{j}, n_{j}>0, j \in \mathrm{I}_{\infty}$ such that

$$
\left|\xi^{-1}-m_{j} / n_{j}\right|<c / n_{j}^{p}
$$

Since $n_{j}>0$ we can order the $n_{j}$ so that they are increasing. Consider $M=\mathrm{M}\left[n_{j} l, N_{j}\right]$ where the $N_{j}$ will be chosen later. By construction $\mathrm{d}_{e^{-l}}(M)<\infty$ and thus $e^{-l} \in \rho(M)$. By lemma $11.7 e^{-\xi l} \in \rho(M)$ if and only if $\sum N_{j} e^{-n_{j} l} \delta_{j}^{2}<\infty$ where

$$
\delta_{j}<n_{j} \xi l\left(c / n_{j}^{p}\right)=c \xi l n_{j}^{-1-\varepsilon} .
$$

Thus we have $e^{-\xi_{l}} \in \rho(M)$ if

$$
\sum N_{j} e^{-n, l} n_{j}^{-2-2 \varepsilon}<\infty .
$$

Now let $\theta$ be any positive irrational number with bounded partial quotients. Then there exists a positive constant $\gamma$ such that

$$
\min _{n_{j}^{\prime}}\left|\theta^{-1}-m_{j}^{\prime} / n_{j}\right|>r / n_{j}^{2+\frac{1}{2} \varepsilon}
$$

where the minimum is taken over all integers $m_{j}^{\prime}$. By lemma 11.7 $e^{-\theta l} \notin \rho(M)$ if and only if $\sum N_{j} e^{-n_{j} l} \delta_{j}(\theta)^{2}=\infty$ where 


$$
\delta_{j}(\theta)>\gamma \theta \ln _{j}^{-1-\frac{1}{2} \varepsilon} .
$$

Thus $e^{-\theta l} \notin \rho(M)$ if

$$
\sum N_{j} e^{-n_{j} l} n_{j}^{-2-\varepsilon}=\infty .
$$

Choose $N_{j}$ so that

$$
2 j^{-1}>N_{j} e^{-n_{j} l} n_{j}^{-2-\varepsilon}>j^{-1}
$$

then Eq. (11.19) is satisfied. Since the $n_{j}$ are strictly increasing we have $n_{j} \geq j$. Thus

$$
N_{j} e^{-n_{j} l} n_{j}^{-2-2 \varepsilon}<2 j^{-1-\varepsilon}
$$

and Eq. (11.16) is satisfied.

Q.E.D.

It is not clear whether or not the algebraic invariant $\rho(M)$ will prove to be a useful tool for the program of classifying all ITPFI factors. Thus it is not known whether or not $\rho\left(M_{1}\right)=\rho\left(M_{2}\right)$ implies $M_{1} \sim M_{2}$, or even whether $\rho(M)=\rho\left(R_{x}\right)$ implies $M \sim R_{x}$, $0<x<1$ (if $x=0,1$ see lemma 11.6). Furthermore it is not clear whether or not all sets $\rho(M)$ allowed by lemma 11.3 actually occur for some $M$, although lemmas 11.8 and 11.10 suggest that lemma 11. 3 may be the only simple general property of $\rho(M)$.

For further classification of an ITPFI $M$, we may use $\mathrm{r}_{\infty}(M \otimes N)$, where $N$ runs over all ITPFI. Again we do not know whether $\mathrm{r}_{\infty}\left(M_{1} \otimes N\right)=\mathrm{r}_{\infty}\left(M_{2} \otimes N\right)$ implies $M_{1}=M_{2}$.

\section{Some Applications}

In this section we determine the isomorphic class of some factors which have been studied previously in the literature [1], [3], [4], $[5],[7],[15],[18]$. In particular we show that certain factors occurring in the quantum theories of infinite free Bose and Fermi systems at a finite temperature are isomorphic to the factor $R_{\infty}$.

We consider first some factors associated with the Fock representation of the canonical commutation relations (CCR's). Let $K$ be a real Hilbert space and let $\mathrm{H}_{\mathrm{F}}(K)$ be the complex Hilbert space 
on which the Fock representation $\mathrm{U}_{\mathrm{F}}(f), \mathrm{V}_{\mathrm{F}}(g), f, g \in K$ of the CCR's over $K$ is defined. Let $K_{1}, K_{2}$ be subspaces (closed linear subsets) of $K$. The von Neumann algebra

$$
\mathrm{R}\left(K_{1}, K_{2} / K\right)=\left\{\mathrm{U}_{\mathrm{F}}(f), \mathrm{V}_{\mathrm{F}}(g) ; f \in K_{1}, g \in K_{2}\right\}^{\prime \prime}
$$

was introduced by Araki [1]. In the following we assume the reader is familiar with the results and notation of [1]. Given $K_{1}, K_{2}$ we define

$$
\begin{aligned}
& K_{4}=K_{1} \cap K_{2}^{\perp} \\
& K_{5}=K_{2} \cap K_{1}^{\perp} \\
& K_{6}=K_{1} \cap K_{2} \\
& K_{7}=K_{4}^{\perp} \cap K_{1} \cap K_{6}^{\perp} \\
& K_{8}=K_{5}^{\perp} \cap K_{2} \cap K_{6}^{\perp} \\
& K_{9}=K_{4}^{\perp} \cap K_{5}^{\perp} \cap K_{6}^{\perp} .
\end{aligned}
$$

The commutant of $\mathrm{R}\left(K_{1}, K_{2} / K\right)$ is $\mathrm{R}\left(K_{2}^{\perp}, K_{1}^{\perp} / K\right)$ and its center is $\mathrm{R}\left(K_{4}, K_{5} / K\right)$. Furthermore it is unitarily equivalent to the tensor product of a maximal abelian algebra $\mathrm{R}\left(K_{4}, K_{5} / K_{4} \oplus K_{5}\right)$, a type I factor $\mathrm{R}\left(K_{6}, K_{6} / K_{6}\right)$, and a factor $\mathrm{R}\left(K_{7}, K_{8} / K_{9}\right)$. Therefore we are interested in the factor $\mathrm{R}\left(K_{1}, K_{2} / K\right)$ when any two of $K_{1}, K_{2}, K_{1}^{\perp}$, $K_{2}^{\perp}$ have zero intersection. In this case there exists a unique closed linear operator $\phi$ from a dense set in $K_{1}$ into $K_{1}^{\perp}$ which is defined by the requirement that the graph of $\phi$ is $K_{2}$ in $K=K_{1} \oplus K_{1}^{\perp}$. It follows from Theorem $2^{\prime}$ of [1] that $\mathrm{R}\left(K_{1}, K_{2} / K\right)$ is then determined up to unitary equivalence by the spectral measure and multiplicity function of the nonnegative selfadjoint operator $\alpha=\phi^{*} \phi$ on $K_{1}$. If the operator $\alpha$ has only a discrete spectrum, then $\mathrm{R}\left(K_{1}, K_{2} / K\right)$ can easily be constructed as an ITPFI factor. It is known that $\mathrm{R}\left(K_{1}, K_{2} / K\right)$ is type I if and only if $\alpha$ is a trace class operator, and that otherwise it is type III [3]. If the spectrum of $\alpha$ is continuous then $\mathrm{R}\left(K_{1}, K_{2} / K\right)$ can be considered as the analog of an ITPFI factor for the continuous tensor product introduced in [2]. In the following we show how the factor $\mathrm{R}\left(K_{1}, K_{2} / K\right)$ can be obtained as the factor generated by a certain reducible representation of the CCR's. 
Let $W$ be a real Hilbert space and let $K=W \oplus W$. The Fock representation of the CCR's over $K$ is given by the equations

$$
\begin{aligned}
& \mathrm{H}_{\mathrm{F}}(K)=\mathrm{H}_{\mathrm{F}}(W) \otimes \mathrm{H}_{\mathrm{F}}(W) \\
& \mathrm{U}_{\mathrm{F}}\left(f_{1} \oplus f_{2}\right)=\mathrm{U}_{\mathrm{F}}\left(f_{1}\right) \otimes \mathrm{U}_{\mathrm{F}}\left(f_{2}\right) \\
& \mathrm{V}_{\mathrm{F}}\left(g_{1} \oplus g_{2}\right)=\mathrm{V}_{\mathrm{F}}\left(g_{1}\right) \otimes \mathrm{V}_{\mathrm{F}}\left(g_{2}\right) .
\end{aligned}
$$

Let $\rho$ be a (possibly unbounded) selfadjoint non-negative operator on $W$. Then the equations

$$
\begin{aligned}
& \mathrm{U}_{\rho}(f)=\mathrm{U}_{\mathrm{F}}\left([1+\rho]^{1 / 2} f\right) \otimes \mathrm{U}_{\mathrm{F}}\left(\rho^{1 / 2} f\right) \\
& \mathrm{V}_{\rho}(g)=\mathrm{V}_{\mathrm{F}}\left([1+\rho]^{1 / 2} f\right) \otimes \mathrm{V}_{\mathrm{F}}\left(-\rho^{1 / 2} g\right)
\end{aligned}
$$

define a reducible representation of the CCR's over the domain $D$ of the operator $\rho^{1 / 2}$ on $W$. The operator algebra

$$
\mathrm{R}(\rho)=\left\{U_{\rho}(f), V_{\rho}(g): f, g \in D\right\}^{\prime \prime}
$$

is a factor (see Sec. 4 of [4]). If we define subspaces of $K$ by

$$
\begin{aligned}
& K_{1}=\left\{f \oplus \rho^{1 / 2}(1+\rho)^{-1 / 2} f: f \in W\right\} \\
& K_{2}=\left\{f \oplus-\rho^{1 / 2}(1+\rho)^{-1 / 2} f: f \in W\right\}
\end{aligned}
$$

then

$$
R(\rho)=R\left(K_{1}, K_{2} / K\right) .
$$

It follows from a straightforward calculation that the operator $\phi$ from $K_{1}$ to $K_{1}^{\perp}$ discussed above is given by

$$
\phi\left(f \oplus \rho^{1 / 2}(1+\rho)^{-1 / 2} f\right)=2 \rho f \oplus-2 \rho^{1 / 2}(1+\rho)^{1 / 2} f
$$

where $f$ is in the domain of $\rho$, and that

$$
\alpha=\phi^{*} \phi=4 \rho(1+\rho) .
$$

It now follows from the above discussion that any factor $R\left(K_{1}, K_{2} / K\right)$ can be obtained as $\mathrm{R}(\rho)$ for some $\rho$.

If the spectrum of $\rho$ is discrete and is given by $\left\{\lambda_{n} ; n \in \mathrm{I}_{\infty}\right\}$ then $\mathrm{R}(\rho)$ can be constructed as an ITPFI factor $\mathrm{R}\left(M_{n}, \Omega_{n}\right)$ where $M_{n}$ is type $\mathrm{I}_{\infty}$ and $\operatorname{Sp}\left(\Omega_{n} / M_{n}\right)=\left\{x_{n}^{k}\left(1-x_{n}\right)^{-1}: k=0,1,2, \cdots\right\}$ where $x_{n}=$ $\lambda_{n}\left(1+\lambda_{n}\right)^{-1}$ (this follows either from Eq. (10.52) of [1] or Eq. (A17) 
of [4]). It follows from lemma 5.10 and definition 3.2 that if $\lambda$ is an accumulation point for the eigenvalues $\lambda_{n}$ then $x \in \mathrm{r}_{\infty}(\mathrm{R}(\rho))$ where $x=\lambda(1+\lambda)^{-1}$. Dell'Antonio [7] has shown that any $\mathrm{R}(\rho)$ is unitarily equivalent to some $\mathrm{R}\left(\rho_{d}\right)$ where $\rho_{d}$ has a discrete spectrum only. In the construction of $[7], \rho_{d}$ satisfies the condition that the operator

$$
\rho^{1 / 2}(1+\rho)^{-1 / 2}-\rho_{d}^{1 / 2}\left(1+\rho_{d}\right)^{-1 / 2}
$$

is Hilbert-Schmidt. It follows that any point $\lambda$ in the continuous spectrum of $\rho$ will be an accumulation point for the eigenvalues of $\rho_{d}$. Thus $\rho$ having a continuous spectrum is a sufficient condition that $\mathrm{R}(\rho) \sim R_{\infty}$.

The representation of the CCR's describing a nonrelativistic infinite free Bose gas at a finite density and finite temperature with no macroscopic occupation of the ground state is of the form $\mathrm{U}_{\rho}(f)$, $\mathrm{V}_{\rho}(g)$ where the operator $\rho$ has a continuous spectrum (see Eqs. (4.10-13), (5.2) of [4]). Thus the von Neumann algebra $\left\{\mathrm{U}_{\rho}(f), \mathrm{V}_{\rho}(g)\right\}^{\prime \prime}$ in this case is the factor $\mathrm{R}_{\infty}$.

Let $\mathrm{U}(f), \mathrm{V}(g)$ be the representation of the CCR's describing a relativistic free Bose field where $f, g$ are suitable functions defined on $R^{3}$. Let $\Delta$ be any open region in $R^{3}$. In the local observables approach to quantum field theory one is interested in the von Neumann algebras

$$
\mathrm{R}(\Delta)=\{\mathrm{U}(f), \mathrm{V}(g) \text { : support } f \subset \Delta\}^{\prime \prime} .
$$

We now construct $R(\Delta)$ as $R\left(K_{1}, K_{2} / K\right)$ where $K$ is the real Hilbert space $L^{2}\left(R^{3}\right)$. We define an unbounded nonnegative selfadjoint operator $\omega$ on $K$ by

$$
(\omega f)(\boldsymbol{k})=\left(k^{2}+m^{2}\right)^{1 / 2} \tilde{f}(\boldsymbol{k}) \quad(m>0)
$$

where $\tilde{f}(\boldsymbol{k})$ is the Fourier transform of $f(\boldsymbol{x})$. The operators $\mathrm{U}(f)$, $\mathrm{V}(g)$ are defined on the Fock space $\mathrm{H}_{\mathrm{F}}(K)$ by

$$
\begin{aligned}
& \mathrm{U}(f)=\mathrm{U}_{\mathrm{F}}\left(\omega^{1 / 2} f\right), f \in D \\
& \mathrm{~V}(g)=\mathrm{V}_{\mathrm{F}}\left(\omega^{-1 / 2} g\right)
\end{aligned}
$$

where $D$ is the domain of $\omega^{1 / 2}$. Given $\Delta \subset R^{3}$ we define 


$$
\begin{aligned}
& K_{0}=\{f \in K: \text { support } f \subset \Delta\} \\
& K_{1}=\left\{\omega^{1 / 2} f: f \in K_{0} \cap D\right\} \\
& K_{2}=\left\{\omega^{-1 / 2} f: f \in K_{0}\right\} .
\end{aligned}
$$

Then $\mathrm{R}(\Delta)=R\left(K_{1}, K_{2} / K\right)$. The operator $\phi$ for this case is

$$
\phi=\left(\omega^{-1 / 2} P \omega^{1 / 2}-1\right) P_{1}
$$

where $P$ is the orthogonal projection on $K_{0}$, and $P_{1}$ is the orthogonal projection on $K_{1}$. Thus $\mathrm{R}(\Delta)$ is determined by the spectral properties of the operator

$$
\alpha=\phi^{*} \phi=P_{1}\left(\omega^{1 / 2} P \omega^{-1 / 2}-1\right)\left(\omega^{-1 / 2} P \omega^{1 / 2}-1\right) P_{1} .
$$

While we have not been able to determine the spectrum of $\alpha$, it seems a reasonable conjecture that $\mathrm{R}(\Delta) \sim R_{\infty}$ for any $\Delta \neq \phi, R^{3}$.

We now consider the factors defined by some representations of the canonical anticommutation relations (CAR's) analogous to the representations of the CCR's defined by Eqs. (12.5), (12.6). We follow the notation of [5]. Let $K$ be a real Hilbert space and let $\mathrm{H}_{J W}(K)$ be the complex Hilbert space on which the no-particle representation of the CAR's over $K$ is defined. Let $\rho$ be a selfadjoint operator on $K$ satisfying $0 \leq \rho \leq 1$. We consider the representation of the CAR's defined by the equations

$$
H=\mathrm{H}_{\mathrm{JW}}(K) \otimes \mathrm{H}_{\mathrm{JW}}(K)
$$

$$
\psi_{\rho}(f)=\psi_{\mathrm{JW}}\left([1-\rho]^{1 / 2} f\right) \otimes 1+\theta_{\mathrm{JW}} \otimes \psi_{\mathrm{JW}}\left(\rho^{1 / 2} f\right) *
$$

(Araki and Wyss [5], Shale and Stinespring [18]). The operator algebra

$$
\mathrm{R}_{A}(\rho)=\left\{\psi(f), \psi r(f)^{*}: f \in K\right\}^{\prime \prime}
$$

is a factor. If the spectrum of $\rho$ is discrete and is given by $\left\{\lambda_{n}: n \in \mathrm{I}_{\infty}\right\}$, then $\mathrm{R}_{A}(\rho)$ can be constructed as an ITPFI factor $\mathrm{R}\left(M_{n}, \Omega_{n}\right)$ where $M_{n}$ is type $\mathrm{I}_{2}$ and $\operatorname{Sp}\left(\Omega_{n} / M_{n}\right)=\left(\lambda_{n}, 1-\lambda_{n}\right)$. It follows that if $\lambda$ is an accumulation point for the eigenvalues $\lambda_{n}$, then $x \in \mathrm{r}_{\infty}\left(R_{A}(\rho)\right)$ where $x=\lambda(1-\lambda)^{-1}$. Dell'Antonio [7] and Rideau [15] have shown that any $\mathrm{R}_{A}(\rho)$ is unitarily equivalent to some $\mathrm{R}_{A}\left(\rho_{d}\right)$ 
where $\rho_{d}$ has a discrete spectrum only. In the construction of $[7], \rho_{d}$ satisfies the condition that the operator

$$
\rho^{1 / 2}(1-\rho)^{-1 / 2}-\rho_{d}^{1 / 2}\left(1-\rho_{d}\right)^{-1 / 2}
$$

is Hilbert-Schmidt. It follows that if $\rho$ has a continuous spectrum the factor $\mathrm{R}_{A}(\rho)$ is the factor $R_{\infty}$.

The representation of the CAR's describing a nonrelativistic infinite free Fermi gas at a finite density and finite temperature is of the form $\psi_{\rho}(f)$ where the operator $\rho$ has a continuous spectrum (see Sec. 12 of [5]). Thus the von Neumann algebra $R_{A}(\rho)$ in this case is again the factor $R_{\infty}$.

\section{Acknowledgement}

Part of this work was carried out during the first named author's visit to the University of Maryland. The first named author would like to thank the members of the Department of Physics and Astronomy for their warm hospitality.

\section{References}

[1] Araki, H.. A lattice of von Neumann algebras associated with the quantum theory of a free Bose field, J. Math. Phys. 4 (1963), 1343-1362.

[2] Araki, H. and E. J. Woods, Complete Boolean algebras of type I factors, Publ. RIMS, Kyoto Univ. Ser. A, 2 (1966), 157-242.

[3] Araki, H., Type of von Neumann algebra associated with free field, Progr. Theoret. Phys. 32 (1964), 956-965.

[4] Araki, H. and E. J. Woods, Representations of the canonical commutation relations describing a nonrelativistic infinite free Bose gas, J. Math. Phys. 4 (1963), 637-662.

[5] Araki, H. and W. Wyss. Representations of canonical anticommutation relations, Helv. Phys. Acta, 37 (1964), 136-159.

[6] Bures, D., Certain factors constructed as infinite tensor products, Comp. Math. 15 (1963), 169-191.

[7] dell' Antonio, G. F., Structure of the algebras of some free systems, Preprint.

[8] Dixmier, J., Les algèbres d'opérateurs dans l'espace hilbertien. Gauthier-Villars, Paris, 1957.

[9] Loève, M., Probability theory, Van Nostrand, New York, 1957.

[10] Moore. C. C., Invariant measures on product spaces. Proceedings of the Fifth Berkeley Symposium on Mathematical Statistics and Probability, vol. II, part 2 (447-459), University of California, Berkeley, 1967. 
[11] Murray, F.J. and J. von Neumann, On rings of operators I, Ann. of Math. 37 (1936), 116-229.

[12] von Neumann. J., On infinite direct products. Comp. Math. 6 (1938), 1-77.

[13] Powers, R. T., Representations of uniformly hyperfinite algebras and their associated von Neumann rings, Ann. of Math. 86 (1967), 138-171.

[14] Pukanszky, L., Some examples of factors, Publ. Math. Debrecen, 4 (1955-56). 135-156.

[15] Rideau, G., On some representations of the anticommutation relations, Preprint.

[16] Sakai, S., On topological properties of $W^{*}$-algebras, Proc. Japan Acad. 33 (1957). 439-444.

[17] Schwartz, J., Two finite, non-hyperfinite, non-isomorphic factors, Comm. Pure Appl. Math. 16 (1963), 19-26.

[18] Shale, D. and W. F. Stinespring, States of the Clifford algebra, Ann. of Math. 80 (1964), 365-381.

[19] Tomita, M., Quasistandard von Neumann algebras, Preprint. 